\title{
Wall-crossings in toric Gromov-Witten theory I: crepant examples
}

\author{
TOM COATES \\ HIROSHI IRITANI \\ HSIAN-HUA TSENG
}

\begin{abstract}
Let $\mathcal{X}$ be a Gorenstein orbifold with projective coarse moduli space $X$ and let $Y$ be a crepant resolution of $X$. We state a conjecture relating the genus-zero GromovWitten invariants of $\mathcal{X}$ to those of $Y$, which differs in general from the Crepant Resolution Conjectures of Ruan and Bryan-Graber, and prove our conjecture when $\mathcal{X}=\mathbb{P}(1,1,2)$ and $\mathcal{X}=\mathbb{P}(1,1,1,3)$. As a consequence, we see that the original form of the Bryan-Graber Conjecture holds for $\mathbb{P}(1,1,2)$ but is probably false for $\mathbb{P}(1,1,1,3)$. Our methods are based on mirror symmetry for toric orbifolds.
\end{abstract}

53D45; 14N35, 83E30

\section{Introduction}

In this paper we use mirror symmetry to determine the relationship between the quantum orbifold cohomology of an orbifold $\mathcal{X}$ and the quantum cohomology of a crepant resolution $Y$ of $\mathcal{X}$ in the cases $\mathcal{X}=\mathbb{P}(1,1,2)$ and $\mathcal{X}=\mathbb{P}(1,1,1,3)$.

\section{A picture from physics}

Quantum cohomology and quantum orbifold cohomology occur in string theory as a small part of a much larger picture. There is supposed to be a moduli space of physical theories-the stringy Kähler moduli space $\mathcal{M}$-and a bundle of algebras over this moduli space formed by the chiral rings of the theories. Near certain limit points of $\mathcal{M}$, called large radius limit points or cusps, the bundle of algebras is given by the quantum cohomology or quantum orbifold cohomology of a target space $\mathcal{X}$; at a general point of $\mathcal{M}$, however, there will be no such description.

More precisely, near each cusp there are distinguished coordinates on $\mathcal{M}$, called flat coordinates, and a distinguished trivialization of the bundle, called a flat trivialization, such that when expressed in flat coordinates and with respect to the flat trivialization the bundle of algebras is isomorphic to the quantum orbifold cohomology algebra 
of $\mathcal{X}$. From this point of view, the quantum parameters $q_{i}$ occurring in the definition of the quantum product ${ }^{1}$ are exponentiated flat coordinates on a neighbourhood of the corresponding cusp in $\mathcal{M}$.

Different cusps can correspond to different target spaces, and in particular the quantum orbifold cohomology $\mathrm{QC}(\mathcal{X})$ of $\mathcal{X}$ and the quantum cohomology $\mathrm{QC}(Y)$ of a crepant resolution $Y$ of $\mathcal{X}$ are expected to come from different cusps of the same moduli space $\mathcal{M}$. Since $\mathrm{QC}(\mathcal{X})$ and $\mathrm{QC}(Y)$ are supposed to be parts of the same global family of algebras this motivates the conjecture, made in various forms by various authors and discussed in detail below, that $\mathrm{QC}(\mathcal{X})$ and $\mathrm{QC}(Y)$ coincide after analytic continuation in quantum parameters. We have already seen, however, the first hint that this conjecture is probably too naïve in general: one should also take into account whether or not the flat coordinates near the cusps associated to $\mathcal{X}$ and to $Y$ coincide after analytic continuation.

\section{Overview of our results}

In what follows we build on work of Givental and Barannikov to construct a rigorous version of this physical picture in the cases $\mathcal{X}=\mathbb{P}(1,1,2)$ and $\mathcal{X}=\mathbb{P}(1,1,1,3)$. Our key tool is (mathematical) mirror symmetry for toric orbifolds. Rather than give a global construction of the stringy Kähler moduli space $\mathcal{M}$-we do not know how to do thiswe instead construct the so-called $B$-model moduli space $\mathcal{M}_{\mathrm{B}}$ and then identify subsets near certain cusps in $\mathcal{M}_{\mathrm{B}}$ with the subsets of $\mathcal{M}$ on which the quantum cohomology of $Y$ and the quantum orbifold cohomology of $\mathcal{X}$ are defined. The B-model moduli space $\mathcal{M}_{\mathrm{B}}$ is expected to coincide under (string theoretic) mirror symmetry with the stringy Kähler moduli space $\mathcal{M}$, but it has the advantage that we can give it rigorous mathematical meaning.

We construct $\mathcal{M}_{\mathrm{B}}$ from the toric data-it is the toric orbifold associated to the secondary fan for the crepant resolution $Y$ of $\mathcal{X}$. Rather than constructing just a family of algebras over $\mathcal{M}_{\mathrm{B}}$ we construct a significantly finer structure called a variation of semi-infinite Hodge structure or VSHS. This VSHS determines, as we will see in Section 2.2 below, a family of algebras over $\mathcal{M}_{\mathrm{B}}$. It also, together with some extra data canonically associated to each cusp, determines flat coordinates and a flat trivialization near each cusp and allows us to compare the flat structures associated to different cusps. The VSHS here consists of a vector bundle $V \rightarrow \mathcal{M}_{\mathrm{B}}$ with flat connection and a family of subspaces $E_{y}, y \in \mathcal{M}_{\mathrm{B}}$, in the fibers of $V$. The vector bundle $V$ is infinitedimensional and the subspaces $E_{y}$ are in an appropriate sense ${ }^{2}$ semi-infinite. The

\footnotetext{
${ }^{1}$ See Section 2.4 below for the definition.

${ }^{2}$ We consider $E_{y}$ to be an element of the Segal-Wilson Grassmannian of $V_{y}$.
} 
family of subspaces $\left\{E_{y}\right\}$ is an analog of a variation of Hodge structure and it satisfies a version of Griffiths transversality-see Section 2.2. The extra data at each cusp consists of an opposite subspace and a dilaton shift. Fix a cusp in $\mathcal{M}_{\mathrm{B}}$ and choose $y$ near that cusp. By parallel transport one can, for each $x \in \mathcal{M}_{\mathrm{B}}$, regard $E_{x}$ as a subspace of the fiber $V_{y}$ and thus define a limiting Hodge structure $E_{\lim } \subset V_{y}$ associated $^{3}$ to the cusp. The opposite subspace associated to the cusp is a subspace $V_{-}$of $V_{y}$ such that $E_{\lim } \oplus V_{-}=V_{y}$, and the dilaton shift is a nonzero element of $E_{\text {lim }}$. The opposite subspace $V_{-}$is uniquely determined by monodromy properties - it is required to be invariant under the local monodromy around the cusp — and a homogeneity condition (Theorem 3.5); $V_{-}$is the analog of the weight filtration on a limiting mixed Hodge structure.

We will see below that when $\mathcal{X}=\mathbb{P}(1,1,2)$, so its crepant resolution $Y$ is the Hirzebruch surface $\mathbb{F}_{2}$, the opposite subspaces at the cusps of $\mathcal{M}_{\mathrm{B}}$ associated to $\mathcal{X}$ and to $Y$ agree under parallel transport in $V$. This implies that the flat structures determined by $\mathcal{X}$ and $Y$ agree: that not only the families of algebras $\mathrm{QC}(\mathcal{X})$ and $\mathrm{QC}(Y)$, but also the flat trivializations and flat coordinates associated to $\mathcal{X}$ and $Y$, are related by analytic continuation. We deduce:

Theorem 1.1 Let $\mathcal{X}=\mathbb{P}(1,1,2)$ and $Y=\mathbb{F}_{2}$. There is a linear isomorphism $\Theta: H_{\text {orb }}^{\bullet}(\mathcal{X} ; \mathbb{C}) \rightarrow H^{\bullet}(Y ; \mathbb{C})$ between the Chen-Ruan orbifold cohomology of $\mathcal{X}$ and the cohomology of $Y$ such that the small quantum orbifold cohomology algebra of $\mathcal{X}$ with quantum parameter $q$ and the small quantum cohomology algebra of $Y$ with quantum parameters $q_{1}, q_{2}$ are isomorphic via $\Theta$, after analytic continuation in $\left(q_{1}, q_{2}\right)$ and the substitution

$$
q_{1}=-1 \quad q_{2}=i \sqrt{q} .
$$

An explicit formula for $\Theta$ is given as Equation (69) below. Furthermore, the map $\Theta$ and the specialization (1) identify the quantum cohomology Frobenius manifolds associated to $\mathcal{X}$ and $Y$.

We will see further that when $\mathcal{X}=\mathbb{P}(1,1,1,3)$, so its crepant resolution $Y$ is the scroll $\mathbb{F}_{3}$, the opposite subspaces at the cusps of $\mathcal{M}_{\mathrm{B}}$ associated to $\mathcal{X}$ and to $Y$ do not agree under parallel transport in $V$. This implies that the flat structures determined by $\mathcal{X}$ and $Y$ are different: they do not agree under analytic continuation. We have:

Theorem 1.2 Let $\mathcal{X}=\mathbb{P}(1,1,1,3)$ and $Y=\mathbb{F}_{3}$. There is a linear isomorphism $\Theta(q)$, which depends nontrivially on $q$, between $H_{\text {orb }}^{\bullet}(\mathcal{X} ; \mathbb{C})$ and $H^{\bullet}(Y ; \mathbb{C})$ such that the

\footnotetext{
${ }^{3}$ Here $E_{\text {lim }}$ is roughly speaking the limit of $E_{x} \subset V_{y}$ as $x$ approaches the cusp.
} 
small quantum orbifold cohomology algebra of $\mathcal{X}$ with quantum parameter $q$ and the small quantum cohomology algebra of $Y$ with quantum parameters $q_{1}, q_{2}$ are isomorphic via $\Theta(q)$, after analytic continuation in $\left(q_{1}, q_{2}\right)$ followed by the substitution

$$
q_{1}=1 \quad q_{2}=\sqrt[3]{q} .
$$

The isomorphism $\Theta(q)$ matches the Poincaré pairing on $H^{\bullet}(Y ; \mathbb{C})$ with the orbifold Poincaré pairing on $H_{\text {orb }}^{\bullet}(\mathcal{X} ; \mathbb{C})$.

An explicit formula for $\Theta(q)$ can be found in Section 3.10. Note that the isomorphism $\Theta(q)$ does not arise from any isomorphism of the quantum cohomology Frobenius manifolds associated to $\mathcal{X}$ and $Y$, as it depends nontrivially on $q$.

\section{Mirror symmetry}

Let us call the VSHS which we construct the $B$-model VSHS. Mirror symmetry identifies the B-model VSHS with an object familiar in Gromov-Witten theory. Givental [28] has observed that if one encodes genus-zero Gromov-Witten invariants of $\mathcal{X}$ in a certain ${ }^{4}$ Lagrangian submanifold $\mathcal{L}_{\mathcal{X}}$ of a symplectic vector space $\mathcal{H}_{\mathcal{X}}$ then many seemingly complicated statements in Gromov-Witten theory are in fact simple geometric assertions about $\mathcal{L}_{\mathcal{X}}$. Mirror symmetry identifies the B-model VSHS with the so-called Amodel VSHS, which consists of the family of tangent spaces to Givental's Lagrangian submanifold $\mathcal{L}_{\mathcal{X}}$. The A-model VSHS is canonically trivialized-it is a family of subspaces of a fixed vector space $\mathcal{H}_{\mathcal{X}}$-and mirror symmetry here asserts that the Bmodel VSHS, expressed with respect to the flat trivialization near the cusp corresponding to $\mathcal{X}$, coincides with the family of tangent spaces to $\mathcal{L}_{\mathcal{X}}$. In the cases at hand this follows from mirror theorems due to Givental [26] and Coates-Corti-Lee-Tseng [15]; it implies in particular that the family of algebra structures over $\mathcal{M}_{\mathrm{B}}$ determined by the B-model VSHS coincides near the cusps of $\mathcal{M}_{\mathrm{B}}$ with the quantum orbifold cohomology of $\mathcal{X}$ and $Y$.

\section{The Crepant Resolution Conjecture}

The results we prove have the following consequence when $\mathcal{X}=\mathbb{P}(1,1,2)$ and $\mathbb{P}(1,1,1,3)$. We conjecture that this holds in general.

\footnotetext{
${ }^{4}$ This encoding is described in Section 2.3 below; here the key point is that knowing $\mathcal{L}_{\mathcal{X}}$ is equivalent to knowing all genus-zero Gromov-Witten invariants of $\mathcal{X}$.
} 
Conjecture 1.3 Suppose that $\mathcal{X}$ is an orbifold with projective coarse moduli space $X$, and that $Y$ is a crepant resolution of $X$. Let $\mathcal{L}_{\mathcal{X}} \subset \mathcal{H}_{\mathcal{X}}$ be Givental's Lagrangian submanifold for $\mathcal{X}$, and let $\mathcal{L}_{Y} \subset \mathcal{H}_{Y}$ be Givental's Lagrangian submanifold for $Y$. Then there exists a linear symplectic isomorphism $\mathbb{U}: \mathcal{H}_{\mathcal{X}} \rightarrow \mathcal{H}_{Y}$, satisfying the conditions enumerated in Conjecture 5.1(a)-(c) below, such that after analytic continuation of $\mathcal{L}_{\mathcal{X}}$ and $\mathcal{L}_{Y}$ we have $\mathbb{U}\left(\mathcal{L}_{\mathcal{X}}\right)=\mathcal{L}_{Y}$

We have not defined $\mathcal{L}_{\mathcal{X}}$ and $\mathcal{L}_{Y}$ at this point, so Conjecture 1.3 is necessarily slightly vague; we give a precise statement as Conjecture 5.1 below. As we will see in Sections 3 and 4 , the symplectic transformation $\mathbb{U}$ here records the effect of parallel transport in the B-model VSHS (ie of parallel transport in the fibers of the vector bundle $V \rightarrow \mathcal{M}_{\mathrm{B}}$ ).

Conjecture 1.3 is our version of the Crepant Resolution Conjecture. We now discuss its relationship to earlier versions of the Crepant Resolution Conjecture formulated by Ruan and by Bryan-Graber. For the rest of this section, let $\mathcal{X}$ be an orbifold with projective coarse moduli space $X$ and let $Y$ be a crepant resolution of $X$.

The first attempt to describe the relationship between the small quantum cohomology algebra of $Y$ and the small quantum orbifold cohomology algebra of $\mathcal{X}$ is due to Ruan. He conjectured that the small quantum orbifold cohomology of $\mathcal{X}$ is isomorphic to the algebra obtained from the small quantum cohomology of $Y$ by analytic continuation in quantum parameters followed by specializing some of those parameters to roots of unity. Theorems 1.1 and 1.2 prove the Ruan Conjecture for $\mathcal{X}=\mathbb{P}(1,1,2)$ and $\mathcal{X}=\mathbb{P}(1,1,1,3)$; the relationship between our Conjecture and the Ruan Conjecture is discussed further in Section 5.4.

A significant strengthening of the Ruan Conjecture has been proposed by BryanGraber [8]. They have modified their conjecture in the light of the examples in this paper, but initially they asserted that the quantum cohomology Frobenius manifolds associated to $\mathcal{X}$ and $Y$ become isomorphic after analytic continuation in quantum parameters. Thus Theorem 1.1 proves the original form of the Bryan-Graber Conjecture for $\mathbb{P}(1,1,2)$, but Theorem 1.2 does not prove the original form of the Bryan-Graber Conjecture for $\mathbb{P}(1,1,1,3)$. In Theorem 5.10 below we show that if the orbifold cohomology of $\mathcal{X}$ satisfies a Hard Lefschetz property - this property holds for $\mathcal{X}=$ $\mathbb{P}(1,1,2)$ but not for $\mathcal{X}=\mathbb{P}(1,1,1,3)$-then our Conjecture implies the original form of the Bryan-Graber Conjecture. In general, however, our Conjecture does not imply the original Bryan-Graber Conjecture and we expect that the latter is false. The most recent version of the Bryan-Graber Conjecture includes the Hard Lefschetz condition as a hypothesis.

We should emphasize that our results here do not show that $\mathcal{X}=\mathbb{P}(1,1,1,3)$ is a counterexample to the original form of the Bryan-Graber Conjecture. It is possible that 
there is a different path of analytic continuation and a different choice of specialization (2) which produces an isomorphism of Frobenius manifolds. But we think that this is unlikely. Conjecture 1.3 expresses the relationship between the quantum cohomology algebras of $\mathcal{X}=\mathbb{P}(1,1,1,3)$ and $Y=\mathbb{F}_{3}$ which is forced upon us by mirror symmetry; in this sense it is the natural conjecture to make. Furthermore the original form of the Bryan-Graber Conjecture ignores some flexibility in parts of the structure- the flat trivialization and flat coordinates - which topological string theory suggests should be background dependent rather than fixed. So we see no compelling reason for the original form of the Bryan-Graber Conjecture to hold. Conjecture 1.3 has been proved in a number of local toric Calabi-Yau examples by Coates [13], and in forthcoming work Iritani [38] will prove it for general toric crepant birational transformations.

\section{Singularity theory}

Our results also have consequences in singularity theory.

We construct the B-model VSHS from a so-called Landau-Ginzburg model. Singularity theorists have long known how to construct the germ of a Frobenius manifold from a Landau-Ginzburg model: for local singularities (germs of isolated hypersurface singularities) this is due to Kyoji Saito [47] and Morihiko Saito [48]; for global singularities (our case) this is due to Douai-Sabbah [19]. It has long been known also that there are in general many possible germs of Frobenius structures for a given singularity: in our language, this is the statement that one can choose from many possible opposite subspaces. From this point of view, the content of this paper is that more global considerations - monodromy and homogeneity properties-single out a canonical opposite subspace associated to each cusp, and that the opposite subspaces associated to different cusps can be compared via analytic continuation.

\section{Plan of the paper}

In Section 2 we fix notation and develop our general theory: we define variations of semi-infinite Hodge structure, introduce Givental's symplectic formalism and explain what we mean by mirror symmetry. In Section 3 we analyze the case $\mathcal{X}=\mathbb{P}(1,1,1,3)$, proving Theorem 1.2 and Conjecture 1.3. The argument which proves Theorem 1.1 and Conjecture 1.3 for $\mathcal{X}=\mathbb{P}(1,1,2)$ is very similar and we summarize it in Section 4 . In Section 5 we describe a more detailed version of Conjecture 1.3 and prove that it implies the most recent form of the Bryan-Graber Conjecture. We conclude with an Appendix describing the Mellin-Barnes method for analytic continuation of hypergeometric functions. 
Acknowledgements This paper was begun whilst the authors held Postdoctoral Fellowships at the Mathematical Sciences Research Institute as part of the "New Topological Structures in Physics" program. We are grateful to MSRI for financial support and for providing such a stimulating research environment and to Yongbin Ruan for many inspiring discussions. This paper forms part of a larger joint project with Alessio Corti $[14 ; 15]$ : we thank him for a productive and enjoyable collaboration and for many useful conversations. We also thank Mina Aganagic, Vincent Bouchard, Alexander Givental and Andrei Mustata for helpful conversations. Tom Coates was partially supported by the Clay Mathematics Institute, the Royal Society and by NSF grant DMS-0401275. Hiroshi Iritani was partially supported by Grant-in-Aid for JSPS Fellows, Scientific Research 18-15108 and EPSRC grant EP/E022162/1. Hsian-Hua Tseng was partially supported by a postdoctoral fellowship at the Pacific Institute for the Mathematical Sciences.

\section{Variations of semi-infinite Hodge structure}

In this section we fix notation for Gromov-Witten invariants, give an introduction to Barannikov's theory of variations of semi-infinite Hodge structure, indicate how this meshes with Givental's geometric approach to Gromov-Witten theory, and explain what we mean by mirror symmetry. We assume that the reader is familiar with quantum cohomology and quantum orbifold cohomology. The quantum cohomology and GromovWitten theory of algebraic varieties have been quite widely studied: good introductions to the field include Fulton-Pandharipande [23], Cox-Katz [18] and Hori et al [33]. The quantum cohomology and Gromov-Witten theory of orbifolds were introduced into mathematics by Chen and Ruan $[11 ; 12]$ in the setting of symplectic geometry; an algebro-geometric version of the theory has been developed by Abramovich, Graber and Vistoli $[1 ; 2]$. An overview of this material, in compatible notation, can be found in Section 2 of Coates-Corti-Lee-Tseng [15]. Givental introduced his formalism in [27] and gave an expository account of it in [28].

\subsection{Notation and conventions}

We work in the algebraic category and over $\mathbb{C}$ : by "manifold" we mean "smooth projective algebraic variety" and we use the terms "orbifold" and "smooth DeligneMumford stack" interchangeably. Introduce notation as follows. 
$\mathcal{X}$

$\mathcal{I X}$

I

$H_{\text {orb }}^{\bullet}(\mathcal{X} ; \mathbb{C})$

age

$(\alpha, \beta)_{\text {orb }}$

$\operatorname{Eff}(\mathcal{X})$

$\langle r\rangle$

$\left\{\phi_{\mu}\right\}_{\mu=1}^{N}$

$\left\{\phi^{\nu}\right\}_{\nu=1}^{N}$ a compact orbifold

the inertia stack of $\mathcal{X}$. A point of $\mathcal{I} \mathcal{X}$ is a pair $(x, g)$ with $x$ a point of $\mathcal{X}$ and $g \in \operatorname{Aut}_{\mathcal{X}}(x)$

the involution of $\mathcal{I} \mathcal{X}$ which sends $(x, g)$ to $\left(x, g^{-1}\right)$

the Chen-Ruan orbifold cohomology groups of $\mathcal{X}$. These are the cohomology groups of the inertia stack

a rational number associated to each component $\mathcal{X}_{i}$ of the inertia stack. The grading on orbifold cohomology is shifted by the age: $\alpha \in H^{p}\left(\mathcal{X}_{i} ; \mathbb{C}\right)$ has degree $\operatorname{deg} \alpha=p+2 \operatorname{age}\left(\mathcal{X}_{i}\right)$

the orbifold Poincaré pairing $\int_{\mathcal{I X}} \alpha \cup I^{\star} \beta$

the set of degrees of representable maps from possibly-stacky curves to $\mathcal{X}$ (ie of degrees of effective curves in $\mathcal{X}$ )

the fractional part $r-\lfloor r\rfloor$ of a rational number $r$

a homogeneous basis for $H_{\text {orb }}^{\bullet}(\mathcal{X} ; \mathbb{C})$

the basis for $H_{\text {orb }}^{\bullet}(\mathcal{X} ; \mathbb{C})$ such that $\left(\phi_{\mu}, \phi^{v}\right)_{\text {orb }}=\delta_{\mu}^{v}$

Example 2.1 Weighted projective space $\mathbb{P}\left(w_{0}, w_{1}, \ldots, w_{n}\right)$ is the stack quotient $\left[\left(\mathbb{C}^{n+1}-\{0\}\right) / \mathbb{C}^{\times}\right]$where $\mathbb{C}^{\times}$acts with weights $-w_{0}, \ldots,-w_{n}$. Components of the inertia stack of $\mathbb{P}\left(w_{0}, \ldots, w_{n}\right)$ are indexed by

$$
F=\left\{\frac{k}{w_{i}} \mid 0 \leq k<w_{i}, 0 \leq i \leq n\right\}
$$

via:

$$
\mathcal{I P}\left(w_{0}, \ldots, w_{n}\right)=\coprod_{f \in F} \mathbb{P}\left(V^{f}\right)
$$

Here

$$
V^{f}=\left\{\left(x_{0}, \ldots, x_{n}\right) \in \mathbb{C}^{n+1} \mid x_{i}=0 \text { unless } w_{i} f \in \mathbb{Z}\right\}
$$

and

$$
\mathbb{P}\left(V^{f}\right)=\left[\left(V^{f}-\{0\}\right) / \mathbb{C}^{\times}\right]
$$

so that $\mathbb{P}\left(V^{f}\right)$ consists of those points of $\mathbb{P}\left(w_{0}, \ldots, w_{n}\right)$ with isotropy group containing $\exp (2 \pi i f) \in \mathbb{C}^{\times}$. The locus $\mathbb{P}\left(V^{f}\right)$ is itself a weighted projective space. The involution $I$ maps the component $\mathbb{P}\left(V^{f}\right)$ to the component $\mathbb{P}\left(V^{\langle-f\rangle}\right)$. The age of $\mathbb{P}\left(V^{f}\right) \subset \mathcal{I}$ is $\left\langle-w_{0} f\right\rangle+\cdots+\left\langle-w_{n} f\right\rangle$.

Example 2.2 The orbifold cohomology of $\mathbb{P}(1,1,2)$ is

$$
H_{\text {orb }}^{\bullet}(\mathbb{P}(1,1,2) ; \mathbb{C})=H^{\bullet}\left(\mathbb{P}\left(V^{0}\right) ; \mathbb{C}\right) \oplus H^{\bullet-2}\left(\mathbb{P}\left(V^{1 / 2}\right) ; \mathbb{C}\right)
$$


where:

$$
\begin{aligned}
\mathbb{P}\left(V^{0}\right) & =\mathbb{P}(1,1,2) & \text { age }=0 \\
\mathbb{P}\left(V^{1 / 2}\right) & =\mathbb{P}(2) & \text { age }=1
\end{aligned}
$$

The involution $I$ is trivial.

Example 2.3 The orbifold cohomology of $\mathbb{P}(1,1,1,3)$ is

$$
\begin{array}{lll}
H_{\text {orb }}^{\bullet}(\mathbb{P}(1,1,1,3) ; \mathbb{C})=H^{\bullet}\left(\mathbb{P}\left(V^{0}\right) ; \mathbb{C}\right) \oplus H^{\bullet-4}\left(\mathbb{P}\left(V^{1 / 3}\right) ; \mathbb{C}\right) \oplus H^{\bullet-2}\left(\mathbb{P}\left(V^{2 / 3}\right) ; \mathbb{C}\right) \\
\text { where: } \\
\qquad \begin{aligned}
\mathbb{P}\left(V^{0}\right) & =\mathbb{P}(1,1,1,3) & & \text { age }=0 \\
\mathbb{P}\left(V^{1 / 3}\right) & =\mathbb{P}(3) & & \text { age }=2 \\
\mathbb{P}\left(V^{2 / 3}\right) & =\mathbb{P}(3) & & \text { age }=1
\end{aligned}
\end{array}
$$

The involution $I$ exchanges $\mathbb{P}\left(V^{1 / 3}\right)$ and $\mathbb{P}\left(V^{2 / 3}\right)$.

2.1.1 Generators and bases for homology and orbifold cohomology We now fix notation for the homology and orbifold cohomology of the spaces which we will consider. When discussing $\mathcal{X}=\mathbb{P}(1,1,2)$ :

$\mathbf{1}_{0} \quad$ is the fundamental class of $\mathbb{P}\left(V^{0}\right)$

$\mathbf{1}_{1 / 2}$ is the fundamental class of $\mathbb{P}\left(V^{1 / 2}\right)$

$p \quad$ is the first Chern class $c_{1}(\mathcal{O}(1)) \in H^{2}(\mathcal{X} ; \mathbb{C})$

and

$$
\begin{array}{llll}
\phi_{0}=\mathbf{1}_{0} & \phi_{1}=p & \phi_{2}=p^{2} & \phi_{3}=\mathbf{1}_{1 / 2} \\
\phi^{0}=2 p^{2} & \phi^{1}=2 p & \phi^{2}=2 \mathbf{1}_{0} & \phi^{3}=2 \mathbf{1}_{1 / 2}
\end{array}
$$

Note that, here and below, $\left(\phi_{i}, \phi^{j}\right)_{\text {orb }}=\delta_{i}^{j}$.

When discussing the Hirzebruch surface $\mathbb{F}_{2}$ which is the projective bundle $\mathbb{P}(\mathcal{O}(-2) \oplus$ $\mathcal{O})$ over $\mathbb{P}^{1}$ :

$p_{1}$ is the class in $H^{2}\left(\mathbb{F}_{2} ; \mathbb{C}\right)$ Poincaré-dual to a fiber

$p_{2}$ is the class in $H^{2}\left(\mathbb{F}_{2} ; \mathbb{C}\right)$ Poincaré-dual to the infinity section

and:

$$
\begin{array}{llll}
\phi_{0}=1 & \phi_{1}=p_{1} & \phi_{2}=p_{2} & \phi_{3}=p_{1} p_{2} \\
\phi^{0}=p_{1} p_{2} & \phi^{1}=p_{2} & \phi^{2}=p_{1} & \phi^{3}=1
\end{array}
$$

The surface $\mathbb{F}_{2}$ is the toric variety corresponding to the fan in Figure 1. 
Figure 1: The fan for the toric variety $\mathbb{F}_{2}$ is the complete fan with these rays.

It can therefore (see for example Audin [4, Chapter VII]) be constructed as the quotient of $\left(\mathbb{C}^{2}-\{0\}\right) \times\left(\mathbb{C}^{2}-\{0\}\right)$ by the action of $\left(\mathbb{C}^{\times}\right)^{2}$

$$
(s, t):\left(\begin{array}{c}
x \\
y \\
z \\
w
\end{array}\right) \longmapsto\left(\begin{array}{c}
s x \\
s y \\
s^{-2} t z \\
t w
\end{array}\right)
$$

and its cohomology ring is

$$
H^{\bullet}\left(\mathbb{F}_{2} ; \mathbb{C}\right)=\mathbb{C}\left[p_{1}, p_{2}\right] /\left\langle p_{1}^{2}, p_{2}^{2}-2 p_{1} p_{2}\right\rangle .
$$

When discussing $\mathcal{X}=\mathbb{P}(1,1,1,3)$ :

$\mathbf{1}_{0}$ is the fundamental class of $\mathbb{P}\left(V^{0}\right)$

$\mathbf{1}_{1 / 3}$ is the fundamental class of $\mathbb{P}\left(V^{1 / 3}\right)$

$\mathbf{1}_{2 / 3}$ is the fundamental class of $\mathbb{P}\left(V^{2 / 3}\right)$

$p \quad$ is the first Chern class $c_{1}(\mathcal{O}(1)) \in H^{2}(\mathcal{X} ; \mathbb{C})$

and: $\quad \phi_{0}=\mathbf{1}_{0} \quad \phi_{1}=p \quad \phi_{2}=p^{2} \quad \phi_{3}=p^{3} \quad \phi_{4}=\mathbf{1}_{1 / 3} \quad \phi_{5}=\mathbf{1}_{2 / 3}$

$$
\phi^{0}=3 p^{3} \quad \phi^{1}=3 p^{2} \quad \phi^{2}=3 p \quad \phi^{3}=31_{0} \quad \phi^{4}=31_{2 / 3} \quad \phi^{5}=31_{1 / 3}
$$

When discussing the projective bundle $\mathbb{F}_{3}=\mathbb{P}(\mathcal{O}(-3) \oplus \mathcal{O})$ over $\mathbb{P}^{2}$ :

$p_{1}$ is the class in $H^{2}\left(\mathbb{F}_{3} ; \mathbb{C}\right)$ Poincaré-dual to the preimage in $\mathbb{F}_{3}$ of a hyperplane in $\mathbb{P}^{2}$

$p_{2}$ is the class in $H^{2}\left(\mathbb{F}_{3} ; \mathbb{C}\right)$ Poincaré-dual to the infinity section 
and:

$$
\begin{aligned}
\phi_{0} & =1 & \phi^{0} & =p_{1}^{2} p_{2} \\
\phi_{1} & =\frac{p_{2}}{3} & \phi^{1} & =p_{1} p_{2} \\
\phi_{2} & =\frac{p_{1} p_{2}}{3} & \phi^{2} & =p_{2} \\
\phi_{3} & =\frac{p_{2}-3 p_{1}}{3} & \phi^{3} & =-p_{1}\left(p_{2}-3 p_{1}\right) \\
\phi_{4} & =-\frac{p_{1}\left(p_{2}-3 p_{1}\right)}{3} & \phi^{4} & =p_{2}-3 p_{1} \\
\phi_{5} & =\frac{p_{1}^{2} p_{2}}{3} & \phi^{5} & =3
\end{aligned}
$$

The scroll $\mathbb{F}_{3}$ is the toric variety corresponding to the fan with rays

$$
e_{1}=\left(\begin{array}{l}
1 \\
0 \\
0
\end{array}\right), e_{2}=\left(\begin{array}{l}
0 \\
1 \\
0
\end{array}\right), e_{3}=\left(\begin{array}{c}
-1 \\
-1 \\
3
\end{array}\right), e_{4}=\left(\begin{array}{l}
0 \\
0 \\
1
\end{array}\right), e_{5}=\left(\begin{array}{c}
0 \\
0 \\
-1
\end{array}\right)
$$

and three-dimensional cones spanned by

$$
\left\{e_{1}, e_{2}, e_{4}\right\},\left\{e_{1}, e_{3}, e_{4}\right\},\left\{e_{2}, e_{3}, e_{4}\right\},\left\{e_{1}, e_{2}, e_{5}\right\},\left\{e_{1}, e_{3}, e_{5}\right\},\left\{e_{2}, e_{3}, e_{5}\right\} \text {. }
$$

It can be constructed as the quotient of $\left(\mathbb{C}^{3}-\{0\}\right) \times\left(\mathbb{C}^{2}-\{0\}\right)$ by the action of $\left(\mathbb{C}^{\times}\right)^{2}$

$$
(s, t):\left(\begin{array}{c}
x \\
y \\
z \\
u \\
v
\end{array}\right) \longmapsto\left(\begin{array}{c}
s x \\
s y \\
s z \\
s^{-3} t u \\
t v
\end{array}\right)
$$

and its cohomology ring is

$$
H^{\bullet}\left(\mathbb{F}_{3} ; \mathbb{C}\right)=\mathbb{C}\left[p_{1}, p_{2}\right] /\left\langle p_{1}^{3}, p_{2}^{2}-3 p_{1} p_{2}\right\rangle .
$$

2.1.2 Gromov-Witten invariants and quantum cohomology We denote GromovWitten invariants using correlators, writing, with notation as in [15]:

$$
\left\langle\alpha_{1} \psi^{k_{1}}, \ldots, \alpha_{n} \psi^{k_{n}}\right\rangle_{g, n, d}^{\mathcal{X}}=\int_{\mathcal{X}_{g, n, d}^{\mathrm{vir}}} \prod_{i=1}^{n} \mathrm{ev}_{i}^{\star} \alpha_{i} \cdot \psi_{i}^{k_{i}}
$$

The integral here means cap product with the virtual fundamental class. If any of the $k_{i}$ are nonzero then (3) is called a gravitational descendant. 
Double correlators denote generating functions for Gromov-Witten invariants:

(4) $\left\langle\left\langle\alpha_{1} \psi^{i_{1}}, \ldots, \alpha_{k} \psi^{i_{k}}\right\rangle_{\tau}^{\mathcal{X}}=\sum_{d \in \operatorname{Eff}(\mathcal{X})} \sum_{n \geq 0} \frac{Q^{d}}{n !}\left\langle\alpha_{1} \psi^{i_{1}}, \ldots, \alpha_{k} \psi^{i_{k}}, \tau, \tau, \ldots, \tau\right\rangle_{0, n+k, d}^{\mathcal{X}}\right.$

where $\tau \in H_{\text {orb }}^{\bullet}(\mathcal{X} ; \mathbb{C})$ and:

$$
Q^{d}= \begin{cases}Q^{\int_{d} p} & \mathcal{X}=\mathbb{P}(1,1,2) \text { or } \mathbb{P}(1,1,1,3) \\ Q_{1}^{\int_{d} p_{1}} Q_{2}^{\int_{d} p_{2}} & \mathcal{X}=\mathbb{F}_{2} \text { or } \mathbb{F}_{3}\end{cases}
$$

These generating functions are formal series in the coordinates $\tau^{1}, \tau^{2}, \ldots, \tau^{N}$ of $\tau=\tau^{1} \phi_{1}+\cdots+\tau^{N} \phi_{N}$ and the variables $Q^{1 / 2}$ or $Q^{1 / 3}$ or $Q_{1}, Q_{2}$. We make these latter variables, which are included to make the series (4) converge, into elements of our ground ring $\Lambda$, setting:

$$
\Lambda= \begin{cases}\mathbb{C} \llbracket Q^{1 / 2} \rrbracket & \text { when } \mathcal{X}=\mathbb{P}(1,1,2) \\ \mathbb{C} \llbracket Q^{1 / 3} \rrbracket & \text { when } \mathcal{X}=\mathbb{P}(1,1,1,3) \\ \mathbb{C} \llbracket Q_{1}, Q_{2} \rrbracket & \text { when } \mathcal{X}=\mathbb{F}_{2} \text { or } \mathcal{X}=\mathbb{F}_{3}\end{cases}
$$

$\Lambda$ is called the Novikov ring. For later use, we define the rings:

$$
\begin{array}{r}
\Lambda\{z\}= \begin{cases}\mathbb{C}[z] \llbracket Q^{1 / 2} \rrbracket & \text { when } \mathcal{X}=\mathbb{P}(1,1,2) \\
\mathbb{C}[z] \llbracket Q^{1 / 3} \rrbracket & \text { when } \mathcal{X}=\mathbb{P}(1,1,1,3) \\
\mathbb{C}[z] \llbracket Q_{1}, Q_{2} \rrbracket & \text { when } \mathcal{X}=\mathbb{F}_{2} \text { or } \mathcal{X}=\mathbb{F}_{3}\end{cases} \\
\Lambda\left\{z, z^{-1}\right\}= \begin{cases}\mathbb{C}\left[z, z^{-1}\right] \llbracket Q^{1 / 2} \rrbracket & \text { when } \mathcal{X}=\mathbb{P}(1,1,2) \\
\mathbb{C}\left[z, z^{-1}\right] \llbracket Q^{1 / 3} \rrbracket & \text { when } \mathcal{X}=\mathbb{P}(1,1,1,3) \\
\mathbb{C}\left[z, z^{-1}\right] \llbracket Q_{1}, Q_{2} \rrbracket & \text { when } \mathcal{X}=\mathbb{F}_{2} \text { or } \mathcal{X}=\mathbb{F}_{3}\end{cases}
\end{array}
$$

The genus-zero descendant potential of $\mathcal{X}$ is

$$
\mathcal{F}_{\mathcal{X}}^{0}\left(t_{0}, t_{1}, \ldots\right)=\sum_{d \in \operatorname{Eff}(\mathcal{X})} \sum_{n \geq 0} \frac{Q^{d}}{n !}\left\langle\mathbf{t}(\psi), \ldots,\left.\mathbf{t}(\psi)\right|_{0, n, d} ^{\mathcal{X}}\right.
$$

where $t_{0}, t_{1}, \ldots$ are orbifold cohomology classes on $\mathcal{X}$ and $\mathbf{t}(\psi)=t_{0}+t_{1} \psi+t_{2} \psi^{2}+\cdots$. This is a formal power series in the coordinates $t_{i}^{\alpha}$ of $t_{i}=t_{i}^{1} \phi_{1}+\cdots+t_{i}^{N} \phi_{N}$ with Taylor coefficients given by genus-zero Gromov-Witten invariants:

$$
\mathcal{F}_{\mathcal{X}}^{0}\left(t_{0}, t_{1}, \ldots\right)=\sum_{\substack{d \in \operatorname{Eff}(\mathcal{X}) \\ n \geq 0}} \sum_{\substack{k_{1}, \ldots, k_{n} \\ \alpha_{1}, \ldots, \alpha_{n}}} \frac{Q^{d} t_{k_{1}}^{\alpha_{1}} \cdots t_{k_{n}}^{\alpha_{n}}}{n !}\left\langle\phi_{\alpha_{1}} \psi^{k_{1}}, \phi_{\alpha_{2}} \psi^{k_{2}}, \ldots,\left.\phi_{\alpha_{n}} \psi^{k_{n}}\right|_{0, n, d} ^{\mathcal{X}}\right.
$$


The big quantum orbifold cohomology of $\mathcal{X}$ is the family of $\Lambda$-algebra structures on $H_{\text {orb }}^{\bullet}(\mathcal{X} ; \Lambda)$ defined by

$$
\left(\phi_{\alpha} \bullet_{\tau} \phi_{\beta}, \phi_{\gamma}\right)_{\text {orb }}=\left\langle\left\langle\phi_{\alpha}, \phi_{\beta}, \phi_{\gamma} \|_{\tau}^{\mathcal{X}}\right.\right.
$$

This family of products $\bullet_{\tau}$ is parametrized by $\tau$ in a formal neighbourhood of zero ${ }^{5}$ in $H_{\text {orb }}^{\bullet}(\mathcal{X} ; \mathbb{C})$. The small quantum orbifold cohomology of $\mathcal{X}$ is a related family of algebra structures on $H_{\text {orb }}^{\bullet}(\mathcal{X} ; \mathbb{C})$ which will be described in detail in Section 2.4. It is defined, roughly speaking, by restricting the parameter $\tau$ in $\bullet_{\tau}$ to lie in $H^{2}(\mathcal{X} ; \mathbb{C}) \subset H_{\text {orb }}^{\bullet}(\mathcal{X} ; \mathbb{C})$.

Remark 2.4 If $\mathcal{X}$ is a manifold then orbifold cohomology, quantum orbifold cohomology, the orbifold Poincaré pairing, and orbifold Gromov-Witten invariants coincide respectively with usual cohomology, usual quantum cohomology, the usual Poincaré pairing, and usual Gromov-Witten invariants.

\subsection{Variations of semi-infinite Hodge structure}

The key notion in this paper is that of a variation of semi-infinite Hodge structure or VSHS. This was introduced by Barannikov [6] as part of his study of higher-dimensional mirror symmetry. VSHSs occur both in the mathematical version of the A-model (quantum cohomology and Gromov-Witten theory) and in the mathematical version of the B-model (singularity theory and Landau-Ginzburg models). As we will see, mirror symmetry in this context amounts to the assertion that the VSHS associated with an A-model is isomorphic to the VSHS associated with its B-model mirror.

A more traditional formulation of mirror symmetry is as the equality of certain families of Frobenius algebras: small quantum cohomology on the A-side and certain Jacobi rings on the B-side. As mentioned in the Introduction, one can obtain a family of Frobenius algebras from a VSHS by choosing an opposite subspace and a dilaton shift. In good cases-in the miniversal situation-a VSHS, an opposite subspace, and a dilaton shift together determine a Frobenius manifold in the sense of Dubrovin [21]; this is also known as a flat structure in the sense of Kyoji Saito [47]. The method of constructing flat structures which we describe here was originally developed by Morihiko Saito [48] in the context of singularity theory, and was reformulated in terms of VSHSs and applied to mirror symmetry by Barannikov [5].

Notation 2.5 Let $\mathbb{C}\left\{z, z^{-1}\right\}$ denote the ring of Laurent power series which converge on $\{z: 0<|z|<\epsilon\}$ for some $\epsilon>0$ which depends on the series under consideration.

\footnotetext{
${ }^{5}$ This just means that the right-hand side of (5) is a formal power series in the coordinates $\tau^{1}, \tau^{2}, \ldots, \tau^{N}$ of $\tau$.
} 
Let $\mathbb{C}\{z\}$ be the subring of $\mathbb{C}\left\{z, z^{-1}\right\}$ consisting of functions regular at $z=0$, and let $\mathcal{O}\left(\mathbb{P}^{1} \backslash\{0\}\right)$ be the ring of holomorphic functions on $\mathbb{P}^{1} \backslash\{0\}$. We have

$$
\mathbb{C}\left\{z, z^{-1}\right\}=\mathbb{C}\{z\} \oplus z^{-1} \mathcal{O}\left(\mathbb{P}^{1} \backslash\{0\}\right)
$$

Let $\left(\mathcal{M}, \mathcal{O}_{\mathcal{M}}\right)$ be a smooth complex analytic space or its formal germ. When $\mathcal{M}$ is a complex analytic space, we define $\mathcal{O}_{\mathcal{M}}\left\{z, z^{-1}\right\}$ to be the sheaf of relative Laurent series in $z$ : for an open set $U \subset \mathcal{M}, \mathcal{O}_{\mathcal{M}}\left\{z, z^{-1}\right\}(U)$ is the set of functions which are holomorphic on $\{(q, z) \in U \times \mathbb{C}: 0<|z|<\epsilon(q)\}$ for some positive continuous map $\epsilon: U \rightarrow \mathbb{R}$ which depends on the function under consideration. Let $\mathcal{O}_{\mathcal{M}}\{z\}$ be the subsheaf of $\mathcal{O}_{\mathcal{M}}\left\{z, z^{-1}\right\}$ consisting of functions regular at $z=0$. When $\left(\mathcal{M}, \mathcal{O}_{\mathcal{M}}\right)$ is a formal germ, we consider Laurent series in $z$ convergent in an adic topology: for a regular parameter system $t^{1}, \ldots, t^{l}$ on $\mathcal{M}$ we set $\mathcal{O}_{\mathcal{M}}\left\{z, z^{-1}\right\}:=\mathbb{C}\left[z, z^{-1}\right] \llbracket t^{1}, \ldots, t^{l} \rrbracket$ and $\mathcal{O}_{\mathcal{M}}\{z\}:=\mathbb{C}[z] \llbracket t^{1}, \ldots, t^{l} \rrbracket$.

Definition 2.6 A variation of semi-infinite Hodge structure (VSHS) with base $\mathcal{M}$ is a locally free $\mathcal{O}_{\mathcal{M}}\{z\}$-module $\mathcal{E}$ of finite rank equipped with a flat $z$-connection ${ }^{6}$

$$
\nabla^{z}: \mathcal{E} \longrightarrow \Omega_{\mathcal{M}}^{1} \otimes \mathcal{O}_{\mathcal{M}} \mathcal{E}
$$

so that

$$
\begin{aligned}
\nabla_{X}^{z}(f(q, z) s) & =(z X f(q, z)) s+f(q, z) \nabla_{X}^{z} s \\
{\left[\nabla_{X}^{z}, \nabla_{Y}^{z}\right] } & =z \nabla_{[X, Y]}^{z}
\end{aligned}
$$

for all $f \in \mathcal{O}_{\mathcal{M}}\{z\}$ and all vector fields $X, Y$ on $\mathcal{M}$, together with a pairing

$$
(\cdot, \cdot): \mathcal{E} \times \mathcal{E} \rightarrow \mathcal{O}_{\mathcal{M}}\{z\}
$$

which satisfies

$$
\begin{aligned}
\left(s_{1}, s_{2}\right)_{\mathcal{E}} & =\left.\left(s_{2}, s_{1}\right)_{\mathcal{E}}\right|_{z \mapsto-z} \\
\left(f(q,-z) s_{1}, s_{2}\right)_{\mathcal{E}} & =\left(s_{1}, f(q, z) s_{2}\right)_{\mathcal{E}}=f(q, z)\left(s_{1}, s_{2}\right)_{\mathcal{E}} \\
z X\left(s_{1}, s_{2}\right)_{\mathcal{E}} & =-\left(\nabla_{X}^{z} s_{1}, s_{2}\right)_{\mathcal{E}}+\left(s_{1}, \nabla_{X}^{z} s_{2}\right)_{\mathcal{E}}
\end{aligned}
$$

for all $f \in \mathcal{O}_{\mathcal{M}}\{z\}$ and all vector fields $X, Y$ on $\mathcal{M}$. The pairing is assumed to be nondegenerate in the sense that the induced pairing

$$
(\mathcal{E} / z \mathcal{E}) \otimes_{\mathcal{O}_{\mathcal{M}}}(\mathcal{E} / z \mathcal{E}) \rightarrow \mathcal{O}_{\mathcal{M}}
$$

${ }^{6} \mathrm{~A} z$-connection is a connection multiplied by $z$. 
is nondegenerate. A grading on this VSHS is a $\mathbb{C}$-endomorphism Gr: $\mathcal{E} \rightarrow \mathcal{E}$ such that there exists a vector field $E$ on $\mathcal{M}$ and a constant $D \in \mathbb{C}$ satisfying

$$
\begin{aligned}
\operatorname{Gr}(f(q, z) s) & =\left(\left(2 z \partial_{z}+2 E\right) f(q, z)\right) s+f(q, z) \operatorname{Gr}(s) \\
{\left[\mathrm{Gr}, \nabla_{X}^{z}\right] } & =2 \nabla_{X}^{z}+\nabla_{[2 E, X]}^{z} \\
\left(2 z \partial_{z}+2 E\right)\left(s_{1}, s_{2}\right)_{\mathcal{E}} & =\left(\operatorname{Gr}\left(s_{1}\right), s_{2}\right)_{\mathcal{E}}+\left(s_{1}, \operatorname{Gr}\left(s_{2}\right)\right)_{\mathcal{E}}-2 D\left(s_{1}, s_{2}\right)_{\mathcal{E}}
\end{aligned}
$$

for all $f \in \mathcal{O}_{\mathcal{M}}\{z\}$ and all vector fields $X$ on $\mathcal{M}$. The vector field $E$, which is uniquely determined by $\mathrm{Gr}$, is called the Euler vector field.

The analogy with a usual variation of Hodge structure comes from the family of filtrations $\cdots \supset z^{-1} \mathcal{E} \supset \mathcal{E} \supset z \mathcal{E} \supset \cdots$ of $\mathcal{E} \otimes_{\mathcal{O}_{\mathcal{M}}\{z} \mathcal{O}_{\mathcal{M}}\left\{z, z^{-1}\right\}$. The existence of the $z$-connection is Griffiths transversality for this family.

Remark 2.7 When defining VSHSs one can choose from many function rings in $z$ : polynomial functions, entire functions, formal power series, $L^{2}\left(S^{1}, \mathbb{C}\right)$, etc. All the VSHSs in our paper can in fact be defined over $\mathbb{C}[z]$. Also, the A-model VSHS is always defined over $\mathbb{C}[z]$. We chose the ring $\mathbb{C}\{z\}$ for technical convenience: it lets us use the Segal-Wilson Grassmannian below.

Suppose that the VSHS $\mathcal{E}$ is generated by one section $s_{0}$ together with its derivatives

$$
\nabla_{X_{1}}^{z} \nabla_{X_{2}}^{z} \cdots \nabla_{X_{k}}^{z} s_{0} \in \mathcal{E}
$$

In this situation $\mathcal{E}$ gives rise to a family of Frobenius algebras over $\mathcal{M}$. Let $T^{*} \mathcal{M}$ be the cotangent bundle of $\mathcal{M}$ and set

$$
\mathcal{O}_{T^{*} \mathcal{M}}:=\bigoplus_{k=0}^{\infty} \operatorname{Sym}^{k}\left(\mathcal{T}_{\mathcal{M}}\right)
$$

where $\mathcal{T}_{\mathcal{M}}$ is the tangent sheaf of $\mathcal{M}$. Then $\mathcal{E} / z \mathcal{E}$ becomes an $\mathcal{O}_{T^{*} \mathcal{M}}$-module via the map

$$
\operatorname{Sym}^{k}\left(\mathcal{T}_{\mathcal{M}}\right) \ni X_{1} X_{2} \cdots X_{k} \longmapsto\left[\nabla_{X_{1}}^{z} \nabla_{X_{2}}^{z} \cdots \nabla_{X_{k}}^{z} s_{0}\right] \in \mathcal{E} / z \mathcal{E}
$$

or in other words

$$
\mathcal{T}_{\mathcal{M}} \ni X \longmapsto\left[\nabla_{X}^{z} \cdot\right] \in \operatorname{End}(\mathcal{E} / z \mathcal{E})
$$

Our assumption implies that there is an exact sequence

$$
0 \longrightarrow \mathcal{I} \longrightarrow \mathcal{O}_{T^{*} \mathcal{M}} \longrightarrow \mathcal{E} / z \mathcal{E} \longrightarrow 0
$$

where $\mathcal{I}$ is an ideal sheaf. This identification of $\mathcal{E} / z \mathcal{E}$ with $\mathcal{O}_{T^{*} \mathcal{M}} / \mathcal{I}$ gives $\mathcal{E} / z \mathcal{E}$ an algebra structure. The pairing $(\cdot, \cdot)$ induces a pairing $\mathcal{E} / z \mathcal{E} \otimes_{\mathcal{O}_{\mathcal{M}}} \mathcal{E} / z \mathcal{E} \rightarrow \mathcal{O}_{\mathcal{M}}$ and 
makes $\mathcal{E} / z \mathcal{E}$ into a Frobenius algebra. Note that the ideal sheaf $\mathcal{I}$ is independent of the choice of generator $s_{0}$, so even though different choices of $s_{0}$ produce different Frobenius algebra structures on $\mathcal{E} / z \mathcal{E}$ they are all isomorphic as algebras.

Definition 2.8 A VSHS is said to be miniversal if there is a section $s_{0}$ of $\mathcal{E}$ such that the $\mathcal{O}_{\mathcal{M}}$-module map

$$
\mathcal{T}_{\mathcal{M}} \ni X \longmapsto\left[\nabla_{X}^{z} s_{0}\right] \in \mathcal{E} / z \mathcal{E}
$$

is an isomorphism. This is equivalent to the natural composition

$$
\mathcal{T}_{\mathcal{M}} \hookrightarrow \mathcal{O}_{T^{*} \mathcal{M}} \rightarrow \mathcal{O}_{T^{*} \mathcal{M}} / \mathcal{I}
$$

being an isomorphism.

In the miniversal case each tangent space $T_{q} \mathcal{M}$ is naturally equipped with a ring structure, independent of the choice of $s_{0}$ above. As we will see below, if we can choose a "good" opposite subspace then this product structure arises from a Frobenius manifold; this implies that $\mathcal{M}$ has the structure of an $F$-manifold ${ }^{7}$ without any choice.

2.2.1 A moving subspace realization As was indicated in the Introduction, the Amodel VSHS arises as a family of subspaces moving in a fixed symplectic vector space $\mathcal{H}_{\mathcal{X}}$. We now explain how to give such a "moving subspace" realization of any VSHS.

Consider the universal cover $\pi: \widetilde{\mathcal{M}} \rightarrow \mathcal{M}$ and let $\mathcal{H}$ denote the space of flat sections of $\pi^{\star}\left(\mathcal{E} \otimes_{\mathcal{O}_{\mathcal{M}}\{z\}} \mathcal{O}_{\mathcal{M}}\left\{z, z^{-1}\right\}\right)$ :

$$
\mathcal{H}=\left\{s \in \Gamma\left(\widetilde{\mathcal{M}}, \pi^{\star}\left(\mathcal{E} \otimes_{\mathcal{O}_{\mathcal{M}}\{z} \mathcal{O}_{\mathcal{M}}\left\{z, z^{-1}\right\}\right)\right): \nabla^{z} s=0\right\}
$$

$\mathcal{H}$ is a free $\mathbb{C}\left\{z, z^{-1}\right\}$-module of the same rank as $\mathcal{E}$. For each $q \in \widetilde{\mathcal{M}}$, we can embed the fiber $\left(\pi^{\star} \mathcal{E}\right)_{q}$ into $\mathcal{H}$ via the map $\iota_{q}$, where

$$
\iota_{q}:\left(\pi^{\star} \mathcal{E}\right)_{q} \ni v \longmapsto s \in \mathcal{H} \text { such that } s(q)=v .
$$

Let $\mathbb{E}_{q} \subset \mathcal{H}$ denote the image of this embedding. Because the pairing $(\cdot, \cdot)$ on $\mathcal{E}$ is $\nabla^{z}$-flat, $\left(s_{1}, s_{2}\right)_{\mathcal{E}}$ is a constant as an element of $\mathbb{C}\left\{z, z^{-1}\right\}$ for any $s_{1}, s_{2} \in \mathcal{H}$. Define a symplectic form $\Omega$ on $\mathcal{H}$ by

$$
\Omega\left(s_{1}, s_{2}\right):=\operatorname{Res}_{z=0}\left(s_{1}, s_{2}\right)_{\mathcal{E}} d z .
$$

${ }^{7}$ See Hertling-Manin [31] or Manin [43] for $F$-manifolds and Coates-Ruan [17, Section 6.2] or Iritani [37, Section 3.2] for an expanded version of this remark. 
In the graded case, the grading operator $\mathrm{Gr}$ induces a $\mathbb{C}$-endomorphism $\mathrm{Gr}$ of $\mathcal{H}$ satisfying

$$
\operatorname{Gr}(f(z) s)=\left(2 z \partial_{z} f(z)\right) s+f(z) \operatorname{Gr}(s)
$$

for all $f \in \mathbb{C}\left\{z, z^{-1}\right\}$ and all $s \in \mathcal{H}$.

We construct our moving subspace realization first in the case where $\mathcal{M}$ is a complex analytic space and the VSHS is analytic. Take a point $q_{0} \in \mathcal{M}$ and fix a basis for $\mathbb{E}_{q_{0}} / z \mathbb{E}_{q_{0}}$ over $\mathbb{C}$. Pick a $\mathbb{C}\{z\}$-basis $e_{1}, \ldots e_{N}$ for $\mathbb{E}_{q_{0}}$ such that $\left[e_{1}\right], \ldots,\left[e_{N}\right]$ is our chosen basis for $\mathbb{E}_{q_{0}} / z \mathbb{E}_{q_{0}}$ and choose local sections $s_{i}$ of $\mathcal{E}$ such that $s_{i}\left(q_{0}\right)=e_{i}\left(q_{0}\right)$. Then for $q$ near $q_{0}$ the subspace $\mathbb{E}_{q}$ is spanned over $\mathbb{C}\{z\}$ by vectors $\iota_{q}\left(s_{i}\right)$ and we can write $\iota_{q}\left(s_{i}\right)=\sum_{j=1}^{N} s_{i j}(q, z) e_{j}$. Let $S(q, z)$ be the matrix with $(i, j)$ entry equal to $s_{i j}(q, z)$. There exists $\epsilon>0$ such that each matrix entry $s_{i j}(q, z)$ converges on $0<|z|<2 \epsilon$, and $S$ therefore defines a $C^{\infty}$ loop $S^{1} \ni z \mapsto S(q, \epsilon z) \in \operatorname{GL}(N, \mathbb{C})$. This loop depends on the choice of local sections $s_{1}, \ldots, s_{N}$. Removing this choice we obtain, after fixing an isomorphism $\mathbb{E}_{q_{0}} / z \mathbb{E}_{q_{0}} \cong \mathbb{C}^{N}$, a holomorphic map from a neighbourhood of $q_{0}$ to the Segal-Wilson Grassmannian $\operatorname{LGL}(N, \mathbb{C}) / \mathrm{L}^{+} \mathrm{GL}(N, \mathbb{C})$ [44]; the choice of isomorphism here is the choice $\left[e_{1}\right], \ldots,\left[e_{N}\right]$ of basis for $\mathbb{E}_{q_{0}} / z \mathbb{E}_{q_{0}}$. At least locally, therefore, the assignment $q \mapsto \mathbb{E}_{q}$ gives an analytic family of elements of the Segal-Wilson Grassmannian. When $\mathcal{M}$ is a formal germ and the VSHS is formal we proceed in the same way, obtaining instead an $\infty$-jet in the Segal-Wilson Grassmannian. With these interpretations in place, we have:

Proposition 2.9 A VSHS with base $\mathcal{M}$ gives a family of subspaces $\mathbb{E}_{q}$ in $\mathcal{H}$ parameterized by $\mathcal{M}$ such that

- $\mathbb{E}_{q}$ is a free $\mathbb{C}\{z\}$-module of dimension equal to the rank of $\mathcal{E}$

- $X \mathbb{E}_{q} \subset z^{-1} \mathbb{E}_{q}$ for any tangent vector $X \in T_{q} \mathcal{M}$

- $\mathbb{E}_{q}$ is maximal isotropic with respect to the symplectic form $\Omega$.

In the graded case we have $(2 E+\mathrm{Gr}) \mathbb{E}_{q} \subset \mathbb{E}_{q}$, so that in particular $\mathrm{Gr} \mathbb{E}_{q} \subset z^{-1} \mathbb{E}_{q}$.

Proof The first three properties are obvious from the definition. Consider the graded case and take a local section $s_{i}(q)=\sum_{j=1}^{N} s_{i j}(q, z) e_{j}(q)$ of $\mathcal{E}$ as above, where $e_{j}(q)$ 
is $\nabla^{z}$-flat: $e_{j}(q)=\iota_{q}^{-1}\left(e_{j}\right)$ for $e_{j} \in \mathcal{H}$. Then:

$$
\begin{aligned}
\iota_{q}\left(\operatorname{Gr}\left(s_{i}\right)\right) & =\sum_{j=1}^{N} \iota_{q}\left(\left(\left(2 E+2 z \partial_{z}\right) s_{i j}(q, z)\right) e_{j}(q)+s_{i j}(q, z) \operatorname{Gr}\left(e_{j}(q)\right)\right) \\
& =\sum_{j=1}^{N}\left(2 E\left(s_{i j}(q, z) e_{j}\right)+\operatorname{Gr}\left(s_{i j}(q, z) e_{j}\right)\right) \\
& =(2 E+\operatorname{Gr}) \iota_{q}\left(s_{i}\right)
\end{aligned}
$$

The left hand side here is an element of $\mathbb{E}_{q}$.

\subsubsection{Opposite subspaces and Frobenius manifolds}

Definition 2.10 Given a VSHS with base $\mathcal{M}$, an opposite subspace at $q \in \widetilde{\mathcal{M}}$ is a free $\mathcal{O}\left(\mathbb{P}^{1} \backslash\{0\}\right)$-submodule $\mathcal{H}_{-}$of $\mathcal{H}$ such that the natural map

$$
\mathcal{H}_{-} \oplus \mathbb{E}_{q} \rightarrow \mathcal{H}
$$

is an isomorphism. This implies that the projections

$$
z \mathcal{H}_{-} / \mathcal{H}_{-} \longleftarrow z \mathcal{H}_{-} \cap \mathbb{E}_{q} \longrightarrow \mathbb{E}_{q} / z \mathbb{E}_{q}
$$

are isomorphisms.

Being opposite to $\mathcal{H}_{-}$is an open condition on $\mathcal{M}$. We can see this using the geometry of the Segal-Wilson Grassmannian. By choosing an opposite subspace $\mathcal{H}_{-}$at $q_{0}$ and an isomorphism $z \mathcal{H}_{-} / \mathcal{H}_{-} \cong \mathbb{C}^{N}$ we can identify each subspace $\mathbb{E}_{q}$ with a point in $\operatorname{LGL}(N, \mathbb{C}) / \mathrm{L}^{+} \mathrm{GL}(N, \mathbb{C})$. Then $\mathcal{H}_{-}$is opposite at $q$ if and only if the point in the Grassmannian corresponding to $\mathbb{E}_{q}$ is in the big cell, and the big cell is an open orbit of $\mathrm{L}^{-} \mathrm{GL}(N, \mathbb{C})$.

An opposite subspace $\mathcal{H}_{-}$at $q_{0} \in \widetilde{\mathcal{M}}$ also defines a trivialization of $\pi^{\star} \mathcal{E}$ near $q_{0}$ :

$$
\left(\pi^{\star} \mathcal{E}\right)_{q} \cong \mathbb{E}_{q} \cong\left(\mathbb{E}_{q} \cap z \mathcal{H}_{-}\right) \otimes \mathbb{C}\{z\} \cong\left(z \mathcal{H}_{-} / \mathcal{H}_{-}\right) \otimes \mathbb{C}\{z\}
$$

for $q$ in some open neighbourhood of $q_{0} \in \widetilde{\mathcal{M}}$. We call this the flat trivialization associated to $\mathcal{H}_{-}$.

Proposition 2.11 Let $\mathcal{H}_{-}$be an opposite subspace at $q_{0} \in \widetilde{\mathcal{M}}$. Under the flat trivialization (8) associated to $\mathcal{H}_{-}$, the flat $z$-connection $\nabla^{z}$ becomes

$$
\nabla_{X}^{z}=z d_{X}+A_{X}
$$


where $d$ is the connection defined by the flat trivialization and $A$ is a $z$-independent $\operatorname{End}\left(z \mathcal{H}_{-} / \mathcal{H}_{-}\right)$-valued $1-$ form on $\widetilde{\mathcal{M}}$. If moreover $\mathcal{H}_{-}$is isotropic with respect to $\Omega$ then the pairing $(\cdot, \cdot)$ induces and can be recovered from the symmetric $\mathbb{C}$-bilinear pairing:

$$
\begin{aligned}
(\cdot, \cdot)_{z \mathcal{H}_{-} / \mathcal{H}_{-}}:\left(z \mathcal{H}_{-} / \mathcal{H}_{-}\right) \otimes & \left(z \mathcal{H}_{-} / \mathcal{H}_{-}\right) \\
{\left[f_{1}\right] \otimes\left[f_{2}\right] } & \left.\longrightarrow\left(f_{1}, f_{2}\right)_{\mathcal{E}}\right|_{z=\infty}
\end{aligned}
$$

This pairing (10) is nondegenerate and satisfies:

$$
\left(A_{X} v, w\right)_{z \mathcal{H}_{-} / \mathcal{H}_{-}}=\left(v, A_{X} w\right)_{z \mathcal{H}_{-} / \mathcal{H}_{-}}
$$

If in addition the VSHS is graded and $\mathcal{H}_{-}$is preserved by the operator Gr then, on $\mathcal{E}$, the operator Gr takes the form

$$
\mathrm{Gr}=2 z \partial_{z}+2 d_{E}+\mathrm{Gr}_{0}
$$

where $\mathrm{Gr}_{0} \in$ End $\left(z \mathcal{H}_{-} / \mathcal{H}_{-}\right)$is a constant operator induced by the action of $\mathrm{Gr}$ on $z \mathcal{H}_{-} \subset \mathcal{H}$. This $\mathrm{Gr}_{0}$ satisfies

$$
2 D(v, w)_{z \mathcal{H}_{-} / \mathcal{H}_{-}}=\left(\mathrm{Gr}_{0}(v), w\right)_{z \mathcal{H}_{-} / \mathcal{H}_{-}}+\left(v, \mathrm{Gr}_{0}(w)\right)_{z \mathcal{H}_{-} / \mathcal{H}_{-}}
$$

where $D$ is the constant from Definition 2.6.

Proof Take a section $s$ of $\mathcal{E}$ which corresponds to a constant vector in $z \mathcal{H}_{-} / \mathcal{H}_{-}$ under the trivialization (8). Then $\iota_{q}(s(q))=v_{0}+w(q)$ for some constant vector $v_{0} \in z \mathcal{H}_{-}$and some $w(q) \in \mathcal{H}_{-}$. Thus $\iota_{q}\left(\nabla_{X}^{z} s(q)\right)=z X \iota_{q}(s(q))=z X w(q)$ is in $\mathbb{E}_{q} \cap z \mathcal{H}_{-}$. This proves (9).

If $\mathcal{H}_{-}$is isotropic with respect to $\Omega$ then it is clear that the restriction of $(\cdot, \cdot)$ to $z \mathcal{H}_{-} \cap \mathbb{E}_{q}$ takes values in $\mathbb{C}$. Under the identification $z \mathcal{H}_{-} \cap \mathbb{E}_{q} \cong z \mathcal{H}_{-} / \mathcal{H}_{-}$, this coincides with the pairing (10). Nondegeneracy follows from the nondegeneracy of the pairing $(\cdot, \cdot)$, and Equation (11) follows from the $\nabla^{z}$-flatness of $(\cdot, \cdot)$.

If $\mathcal{H}_{-}$is preserved by $\mathrm{Gr}$ then so is $z \mathcal{H}_{-}$, and thus $\mathrm{Gr}$ induces a constant operator $\mathrm{Gr}_{0} \in \operatorname{End}\left(z \mathcal{H}_{-} / \mathcal{H}_{-}\right)$; equations (12) and (13) follow immediately from (6).

In the miniversal and graded case, the structures in Proposition 2.11 assemble to give a Frobenius manifold with base an open subset of $\widetilde{\mathcal{M}}$. Take an $\Omega$-isotropic, $\mathrm{Gr}$-invariant opposite subspace $\mathcal{H}_{-}$. Assume that there exists an eigenvector $\left[v_{0}\right] \in z \mathcal{H}_{-} / \mathcal{H}_{-}$of $\mathrm{Gr}_{0}$ such that the corresponding section $s_{0} \in \mathcal{E}$ under the trivialization (8) makes (7) into 
an isomorphism. The eigenvector $\left[v_{0}\right]$ is called the dilaton shift. The affine subspace $v_{0}+\mathcal{H}_{-}$meets each $\mathbb{E}_{q}$ in a unique point $\iota_{q}\left(s_{0}\right)$. Barannikov's period map is

$$
\begin{aligned}
\Psi: \widetilde{\mathcal{M}} & \longrightarrow \mathcal{H} \\
q & \longmapsto \iota_{q}\left(s_{0}(q)\right) .
\end{aligned}
$$

The miniversality condition implies that the map

$$
\begin{aligned}
\widetilde{\mathcal{M}} & \longrightarrow z \mathcal{H}_{-} / \mathcal{H}_{-} \\
q & \longmapsto\left[z\left(\Psi(q)-v_{0}\right)\right]
\end{aligned}
$$

is a local isomorphism. The linear coordinates on $\widetilde{\mathcal{M}}$ given by this map are called flat coordinates. By differentiating (15) we obtain the Kodaira-Spencer map:

$$
\mathrm{KS}: \mathcal{T}_{\widetilde{\mathcal{M}}} \ni X \longmapsto A_{X}\left[v_{0}\right] \in z \mathcal{H}_{-} / \mathcal{H}_{-}
$$

Pulling back the metric $(\cdot, \cdot)_{z \mathcal{H}_{-} / \mathcal{H}_{-}}$on $z \mathcal{H}_{-} / \mathcal{H}_{-}$along KS gives a nondegenerate symmetric bilinear pairing:

$$
g: \mathcal{T}_{\widetilde{\mathcal{M}}} \otimes \mathcal{T}_{\widetilde{\mathcal{M}}} \rightarrow \mathcal{O}_{\widetilde{\mathcal{M}}}
$$

This is constant with respect to the flat coordinates, so the "flat coordinates" really are flat coordinates for the metric ${ }^{8} g$. The product $\bullet$ on tangent spaces is defined by

$$
A_{X \bullet Y}\left[v_{0}\right]=A_{X} A_{Y}\left[v_{0}\right] .
$$

The identity vector field $e$ is given by

$$
A_{e}\left[v_{0}\right]=\left[v_{0}\right]
$$

It is easy to check that these data satisfy all the axioms for a Frobenius manifold:

Proposition 2.12 Take an $\Omega$-isotropic, $\mathrm{Gr}$-invariant opposite subspace $\mathcal{H}_{-}$and a dilaton shift $\left[v_{0}\right] \in z \mathcal{H}_{-} / \mathcal{H}_{-}$such that the corresponding section $s_{0} \in \mathcal{E}$ under the trivialization (8) makes (7) into an isomorphism. Then the data $(\bullet, e, g, E)$ defined above determine a Frobenius manifold with base an open subset of $\widetilde{\mathcal{M}}$. In other words:

(1) The Levi-Civita connection $\nabla^{\mathrm{LC}}$ of $g$ is flat.

(2) $\left(T_{q} \mathcal{M}, \bullet, g\right)$ is an associative, commutative Frobenius algebra.

(3) The pencil of connections $\nabla_{X}^{\lambda}=\nabla_{X}^{\mathrm{LC}}+\lambda X \bullet$ is flat.

(4) The identity vector field $e$ is flat.

\footnotetext{
${ }^{8}$ Note that the metric $g$ is a $\mathbb{C}$-bilinear form not a Hermitian form.
} 
(5) The Euler vector field satisfies $\left(\nabla^{\mathrm{LC}}\right)^{2} E=0$ and

$$
\begin{aligned}
E g(X, Y) & =g([E, X], Y)+g(X,[E, Y])+(\alpha+2-D) g(X, Y), \\
{[E, X \bullet Y] } & =[E, X] \bullet Y+X \bullet[E, Y]+[X, Y]
\end{aligned}
$$

where $\alpha$ is the eigenvalue of $\left[v_{0}\right]$ with respect to $\mathrm{Gr}_{0}$.

In these terms, $\mathrm{Gr}_{0}$ is given by

$$
\mathrm{Gr}_{0}(\mathrm{KS}(X))=\mathrm{KS}\left((2+\alpha) X-2 \nabla_{X}^{\mathrm{LC}} E\right) .
$$

Remark 2.13 Even in the nonminiversal case, if the map (15) is injective with image an affine subspace of $z \mathcal{H}_{-} / \mathcal{H}_{-}$then we still refer to the linear coordinates on $\widetilde{\mathcal{M}}$ given by (15) as flat coordinates. In this case the pullback of the pairing on $z \mathcal{H}_{-} / \mathcal{H}_{-}$ via the Kodaira-Spencer map will not in general give a metric on the base $\widetilde{\mathcal{M}}$. But these "flat coordinates" and the constant pairing on $z \mathcal{H}_{-} / \mathcal{H}_{-}$are what would remain from the flat structure on the Frobenius manifold if our nonminiversal VSHS arose as a "slice" of a miniversal VSHS. This is exactly the relationship between small and big quantum cohomology.

\subsection{The big A-model VSHS and Givental's symplectic formalism}

We now define a VSHS which gives rise, through an appropriate choice of opposite subspace and dilaton shift, to the Frobenius manifold structure on big quantum orbifold cohomology. This is the big A-model VSHS described in the Introduction. As we will see below, the moving subspace realization of this VSHS gives the family of tangent spaces to Givental's Lagrangian cone.

2.3.1 The big A-model VSHS Let $H$ be a formal neighbourhood of zero in $H_{\text {orb }}^{\bullet}(\mathcal{X}, \Lambda)$, where $\Lambda$ and associated rings are defined in Section 2.1.2. Recall that, for a linear coordinate system $\tau^{1}, \ldots, \tau^{N}$ on $H_{\text {orb }}^{\bullet}(\mathcal{X}, \mathbb{C})$, we have:

$$
\begin{aligned}
& \mathcal{O}_{H}=\Lambda \llbracket \tau^{1}, \ldots, \tau^{N} \rrbracket \\
& \mathcal{O}_{H}\{z\}=\Lambda\{z\} \llbracket \tau^{1}, \ldots, \tau^{N} \rrbracket \\
& \mathcal{O}_{H}\left\{z, z^{-1}\right\}=\Lambda\left\{z, z^{-1}\right\} \llbracket \tau^{1}, \ldots, \tau^{N} \rrbracket
\end{aligned}
$$

The big A-model VSHS has base $H$ and is given by:

$$
\mathcal{E}_{\mathrm{A}}^{\mathrm{big}}:=H_{\mathrm{orb}}^{\bullet}(\mathcal{X}, \mathbb{C}) \otimes \mathcal{O}_{H}\{z\}
$$


The flat $z$-connection is the Dubrovin connection

$$
\nabla^{z}=z d+\sum_{\alpha=1}^{N}\left(\phi_{\alpha} \bullet_{\tau}\right) d \tau^{\alpha},
$$

the pairing is

$$
\begin{aligned}
\mathcal{E}_{\mathrm{A}}^{\mathrm{big}} \times \mathcal{E}_{\mathrm{A}}^{\mathrm{big}} & \longrightarrow \mathcal{O}_{H}\{z\} \\
f(z) \times g(z) & \longmapsto(f(-z), g(z))_{\text {orb }}
\end{aligned}
$$

and the grading operator Gr: $\mathcal{E}_{\mathrm{A}}^{\mathrm{big}} \rightarrow \mathcal{E}_{\mathrm{A}}^{\mathrm{big}}$ and Euler vector field $E$ are

$$
\begin{aligned}
\mathrm{Gr} & =2 z \partial_{z}+2 d_{E}+\mathrm{Gr}_{0} \\
E & =\sum_{\alpha}\left(1-\frac{\operatorname{deg} \phi_{\alpha}}{2}\right) \tau^{\alpha} \frac{\partial}{\partial \tau^{\alpha}}+\sum_{\alpha: \operatorname{deg} \phi_{\alpha}=2} \rho_{\alpha} \frac{\partial}{\partial \tau^{\alpha}}
\end{aligned}
$$

where $\mathrm{Gr}_{0}$ is the usual grading operator on orbifold cohomology

$$
\operatorname{Gr}_{0}\left(\phi_{\alpha}\right)=\operatorname{deg}\left(\phi_{\alpha}\right) \phi_{\alpha}
$$

and $c_{1}(\mathcal{X})=\sum_{\alpha: \operatorname{deg} \phi_{\alpha}=2} \rho_{\alpha} \phi_{\alpha}$. These data satisfy the axioms for a graded VSHS (Definition 2.6) with $D=\operatorname{dim}_{\mathbb{C}} \mathcal{X}$, except that the ground ring $\mathbb{C}$ there is replaced here by $\Lambda$.

2.3.2 Givental's symplectic formalism Following Givental [28], we now describe the genus-zero Gromov-Witten theory of $\mathcal{X}$ in terms of a Lagrangian submanifold $\mathcal{L}_{\mathcal{X}}$ of the symplectic vector space

$$
\begin{aligned}
\mathcal{H}_{\mathcal{X}} & :=H_{\text {orb }}^{\bullet}(\mathcal{X} ; \mathbb{C}) \otimes \Lambda\left\{z, z^{-1}\right\} & & \text { (the vector space) } \\
\Omega(f, g) & :=\operatorname{Res}_{z=0}(f(-z), g(z))_{\text {orb }} d z & & \text { (the symplectic form). }
\end{aligned}
$$

Relations between genus-zero Gromov-Witten invariants-the String Equation, Dilaton Equation, and Topological Recursion Relations - translate into very strong constraints on the geometry of $\mathcal{L}_{\mathcal{X}}$; see Coates-Givental [16, Proposition 1], Givental [28] and Coates-Corti-Iritani-Tseng [14, Appendix B]. These constraints can be rephrased as the statement that the tangent spaces to $\mathcal{L}_{\mathcal{X}}$ form the moving subspace realization of a VSHS; we will see in the next section that this is the big A-model VSHS.

The space $\mathcal{H}_{\mathcal{X}}$ is the direct sum of Lagrangian subspaces

$$
\mathcal{H}_{\mathcal{X}}^{+}=H_{\text {orb }}^{\bullet}(\mathcal{X} ; \mathbb{C}) \otimes \Lambda\{z\}, \quad \mathcal{H}_{\mathcal{X}}^{-}=z^{-1} H_{\text {orb }}^{\bullet}(\mathcal{X} ; \mathbb{C}) \otimes \Lambda\left\{z^{-1}\right\}
$$


and this polarization identifies the symplectic space $\left(\mathcal{H}_{\mathcal{X}}, \Omega\right)$ with the cotangent bundle $T^{\star} \mathcal{H}_{\mathcal{X}}^{+}$. We will regard the genus-zero descendant potential $\mathcal{F}_{\mathcal{X}}^{0}$ as the formal germ of a function on $\mathcal{H}_{\mathcal{X}}^{+}$. A general point of $\mathcal{H}_{\mathcal{X}}^{+}$takes the form

$$
\mathbf{q}(z)=q_{0}+q_{1} z+q_{2} z^{2}+\cdots
$$

where $q_{0}, q_{1}, \ldots$ are orbifold cohomology classes on $\mathcal{X}$. Setting

$$
q_{k}= \begin{cases}t_{k} & k \neq 1 \\ t_{1}-1 & k=1\end{cases}
$$

makes $\mathcal{F}_{\mathcal{X}}^{0}$ into a function on a formal neighbourhood ${ }^{9}$ of the point $-z$ in $\mathcal{H}_{\mathcal{X}}^{+}$. This change of variables $\mathbf{q}(z)=\mathbf{t}(z)-z$ is called the dilaton shift.

The Lagrangian submanifold $\mathcal{L}_{\mathcal{X}}$ is the graph of the differential of $\mathcal{F}_{\mathcal{X}}^{0}$. Since $\mathcal{F}_{\mathcal{X}}^{0}$ is defined only in a formal neighbourhood of $-z \in \mathcal{H}_{\mathcal{X}}^{+}, \mathcal{L}_{\mathcal{X}}$ is a formal germ of a Lagrangian submanifold of $T^{\star} \mathcal{H}_{\mathcal{X}}^{+}$. The polarization $\mathcal{H}_{\mathcal{X}}=\mathcal{H}_{\mathcal{X}}^{+} \oplus \mathcal{H}_{\mathcal{X}}^{-}$identifies $T^{\star} \mathcal{H}_{\mathcal{X}}^{+}$with $\mathcal{H}_{\mathcal{X}}$, and we regard $\mathcal{L}_{\mathcal{X}}$ as a formal germ of a Lagrangian submanifold of $\mathcal{H}_{\mathcal{X}}$ via this identification. $\mathcal{L}_{\mathcal{X}}$ has a more concrete description as follows. A general point of $\mathcal{H}_{\mathcal{X}}$ has the form

$$
\sum_{k=0}^{\infty} \sum_{\mu=1}^{N} q_{k}^{\mu} \phi_{\mu} z^{k}+\sum_{l=0}^{\infty} \sum_{\nu=1}^{N} p_{l, \nu} \phi^{\nu}(-z)^{-1-l}
$$

where $\phi^{1}, \ldots, \phi^{N}$ is the basis for $H_{\text {orb }}^{\bullet}(\mathcal{X} ; \mathbb{C})$ such that $\left(\phi_{i}, \phi^{j}\right)_{\text {orb }}=\delta_{i}{ }^{j}$, and this defines Darboux coordinates $\left\{q_{k}^{\mu}, p_{l, \nu}\right\}$ on $\mathcal{H}_{\mathcal{X}}$. In these coordinates $\mathcal{L}_{\mathcal{X}}$ is given by

$$
p_{l, v}=\frac{\partial \mathcal{F}_{\mathcal{X}}^{0}}{\partial q_{l}^{v}}
$$

so a general point on $\mathcal{L}_{\mathcal{X}}$ takes the form:

$$
-z+\mathbf{t}(z)+\sum_{\substack{d \in \operatorname{Eff}(\mathcal{X}) \\ n \geq 0}} \sum_{\alpha=1}^{N} \frac{Q^{d}}{n !}\left\langle\mathbf{t}(\psi), \ldots, \mathbf{t}(\psi),\left.\frac{\phi_{\alpha}}{-z-\psi}\right|_{0, n+1, d} ^{\mathcal{X}} \phi^{\alpha}\right.
$$

The expression $1 /(-z-\psi)$ here should be expanded as a power series in $z^{-1}$. Note that $\mathcal{L}_{\mathcal{X}}$ encodes all genus-zero Gromov-Witten invariants of $\mathcal{X}$.

${ }^{9}$ This just means that $\mathcal{F}_{\mathcal{X}}^{0}$ is a formal power series in the variables $t_{k}^{\alpha}$. 
Turning off gravitational descendants, by setting

$$
t_{k}= \begin{cases}\tau & k=0 \\ 0 & k \neq 0\end{cases}
$$

gives a family $\tau \mapsto J_{\mathcal{X}}(\tau,-z)$ of elements of $\mathcal{L}_{\mathcal{X}}$ parametrized by $H$. We call this the big $J$-function of $\mathcal{X}$. From (17) we have:

$$
J_{\mathcal{X}}(\tau,-z)=-z+\tau+\sum_{\alpha=1}^{N} \|\left\langle\frac{\phi^{\alpha}}{-z-\psi} \|_{\tau}^{\mathcal{X}} \phi_{\alpha}\right.
$$

As discussed, $\mathcal{L}_{\mathcal{X}}$ has a very special geometric structure. The precise geometric properties we need are given in Proposition 2.14 below. These imply that $\mathcal{L}_{\mathcal{X}}$ is the germ of a Lagrangian cone with vertex at the origin such that each tangent space $T$ is tangent to the cone exactly along $z T$. Define a submersion $\hat{\tau}: \mathcal{L}_{\mathcal{X}} \rightarrow H$ by

$$
\hat{\tau}(\mathbf{q}, \mathbf{p})=\sum_{d \in \operatorname{Eff}(\mathcal{X})} \sum_{n \geq 0} \sum_{\alpha=1}^{N} \frac{Q^{d}}{n !}\left\langle 1, \mathbf{t}(\psi), \mathbf{t}(\psi), \ldots, \mathbf{t}(\psi),\left.\phi^{\alpha}\right|_{0, n+2, d} ^{\mathcal{X}} \phi_{\alpha} .\right.
$$

Here we used the dilaton shift $\mathbf{q}(z)=\mathbf{t}(z)-z$ as before. The String Equation shows that $\hat{\tau}\left(J_{\mathcal{X}}(\tau,-z)\right)=\tau$, ie that the $J$-function is a section of the map $\hat{\tau}: \mathcal{L}_{\mathcal{X}} \rightarrow H$. Denote by $T_{\tau}$ the tangent space to $\mathcal{L}_{\mathcal{X}}$ at $J_{\mathcal{X}}(\tau,-z)$.

\section{Proposition 2.14}

(a) The tangent space to $\mathcal{L}_{\mathcal{X}}$ at $(\mathbf{q}, \mathbf{p})$ coincides with $T_{\tau}$ for $\tau=\widehat{\tau}(\mathbf{q}, \mathbf{p})$.

(b) The tangent space $T_{\tau}$ is closed under multiplication by $z$ and has the structure of a $\Lambda\{z\}$-module. Moreover, it is freely generated over $\Lambda\{z\}$ by the partial derivatives

$$
\frac{\partial}{\partial \tau^{1}} J_{\mathcal{X}}(\tau,-z), \ldots, \frac{\partial}{\partial \tau^{N}} J_{\mathcal{X}}(\tau,-z) .
$$

(c) The fiber at $\tau \in H$ of the map $\hat{\tau}: \mathcal{L}_{\mathcal{X}} \rightarrow H$ is given by

$$
z T_{\tau} \cap\left(\mathcal{H}_{\mathcal{X}},-z\right)
$$

where $\left(\mathcal{H}_{\mathcal{X}},-z\right)$ is the formal neighborhood ${ }^{10}$ of $-z$ in $\mathcal{H}_{\mathcal{X}}$.

${ }^{10}$ See $\left[14\right.$, Appendix B] for the definition of $\left(\mathcal{H}_{\mathcal{X}},-z\right)$ as a formal scheme over $\Lambda$. The intersection here should be interpreted as the set of $R$-valued points for an arbitrary $\Lambda$-algebra $R$ when $\tau$ is an $R$-valued point of $H$. 
Proof Part (a) is [14, Lemma B6] and part (b) is [14, Proposition B3]. We sketch the proof of (c) following Givental [28]. Take $\mathbf{f} \in \hat{\tau}^{-1}(\tau)$. The String Equation says that $z^{-1} \mathbf{f} \in T_{\mathbf{f}} \mathcal{L}_{\mathcal{X}}$. Thus $\mathbf{f} \in z T_{\mathbf{f}} \mathcal{L}_{\mathcal{X}}=z T_{\tau}$ by (a), and so $\hat{\tau}^{-1}(\tau) \subset z T_{\tau}$. In particular $\operatorname{Ker} d_{\mathbf{f}} \hat{\tau} \subset z T_{\tau}$. Both $\operatorname{Ker} d_{\mathbf{f}} \hat{\tau}$ and $z T_{\tau}$ are subspaces of $T_{\tau}$ and, as $\hat{\tau}$ is submersive, they each have codimension equal to $\operatorname{dim} H$. Thus $\operatorname{Ker} d_{\mathbf{f}} \hat{\tau}=z T_{\tau}$. Part (c) follows. $\square$

Part (c) of this proposition shows that $\mathcal{L}_{\mathcal{X}}$ can be reconstructed from its tangent spaces as

$$
\mathcal{L}_{\mathcal{X}}=\bigcup_{\tau \in H}\left(z T_{\tau} \cap\left(\mathcal{H}_{\mathcal{X}},-z\right)\right)
$$

2.3.3 The big A-model VSHS and Givental's cone Consider the $\mathcal{O}_{H}\{z\}$-linear map:

$$
\begin{aligned}
\mathbb{J}: \mathcal{E}_{\mathrm{A}}^{\mathrm{big}} & \longrightarrow H_{\mathrm{orb}}^{\bullet}(\mathcal{X}, \Lambda) \otimes \mathcal{O}_{H}\left\{z, z^{-1}\right\} \\
\phi_{\alpha} & \longmapsto \frac{\partial}{\partial \tau^{\alpha}} J_{\mathcal{X}}(\tau, z)
\end{aligned}
$$

Using the well-known relationship between the $J$-function and the big quantum product

$$
z \frac{\partial}{\partial \tau^{\alpha}} \frac{\partial}{\partial \tau^{\beta}} J_{\mathcal{X}}(\tau, z)=\sum_{\gamma=1}^{N}\left\langle\left\langle\phi_{\alpha}, \phi_{\beta}, \phi^{\gamma} \|_{\tau}^{\mathcal{X}} \frac{\partial}{\partial \tau^{\gamma}} J_{\mathcal{X}}(\tau, z)\right.\right.
$$

we see that $\mathbb{J}$ satisfies

$$
\mathbb{J} \circ \nabla_{X}^{z}=z d_{X} \circ \mathbb{J}
$$

for any vector field $X$ on $H$. Thus $\mathbb{J}$ sends any $\nabla^{z}$-parallel section to a constant section: it identifies the space of $\nabla^{z}$-flat sections of the big A-model VSHS-which we denoted by $\mathcal{H}$ in Section 2.2.1-with Givental's symplectic space $\mathcal{H}_{\mathcal{X}}$. Proposition 2.14(b) implies that the image of each fiber $\mathcal{E}_{\mathrm{A}, \tau}^{\mathrm{big}}$ under $\mathbb{J}$ coincides, if we flip the sign of $z$, with the tangent space $T_{\tau}$ to $\mathcal{L}_{\mathcal{X}}$ :

$$
\mathbb{E}_{\tau}(\Lambda):=\mathbb{J}\left(\mathcal{E}_{\mathrm{A}, \tau}^{\mathrm{big}}\right)=\left.T_{\tau}\right|_{z \mapsto-z}
$$

So the moving subspace realization $\mathbb{E}_{\tau}(\Lambda)$ of the big A-model VSHS determines the Lagrangian submanifold $\mathcal{L}_{\mathcal{X}}$ via (19). Since $\mathbb{J} \circ \mathrm{Gr}=\left(2 z \partial_{z}+2 d_{E}+\mathrm{Gr}_{0}-2 c_{1}(\mathcal{X}) / z\right) \circ \mathbb{J}$, we see that the grading operator acts on $\mathcal{H}_{\mathcal{X}}$ by:

$$
\left.\mathrm{Gr}\right|_{\mathcal{H}_{\mathcal{X}}}=2 z \partial_{z}+\mathrm{Gr}_{0}-2 c_{1}(\mathcal{X}) / z
$$

The standard opposite subspace for the big A-model VSHS is $\mathcal{H}_{\mathcal{X}}^{-}$. This is clearly isotropic with respect to $\Omega$; it is also preserved by Gr. The period map (14) associated 
to this opposite subspace and the choice $\left[v_{0}\right]=1 \in z \mathcal{H}_{\mathcal{X}}^{-} / \mathcal{H}_{\mathcal{X}}^{-}$(the dilaton shift) is $\tau \mapsto z^{-1} J_{\mathcal{X}}(\tau, z)$ :

$$
\mathbb{E}_{\tau}(\Lambda) \cap\left(1+\mathcal{H}_{\mathcal{X}}^{-}\right)=\left\{z^{-1} J_{\mathcal{X}}(\tau, z)\right\}
$$

Since $J(\tau, z)=z+\tau+O\left(z^{-1}\right)$, the flat coordinates here are the usual linear coordinates $\tau^{i}$ on $H$. The big A-model VSHS is miniversal, and these choices of opposite subspace $\mathcal{H}_{\mathcal{X}}^{-}$and dilaton shift $\left[v_{0}\right]$ produce the usual big quantum orbifold cohomology Frobenius manifold defined by Chen-Ruan [11].

2.3.4 The analytic big A-model VSHS and Givental's cone over $\mathbb{C}$ Suppose that the big quantum product $\bullet_{\tau}$ in (5) is convergent as a power series in $\tau$ and $Q$. Then we can consider the big A-model VSHS and the Lagrangian submanifold over $\mathbb{C}$ by specializing all the Novikov variables $Q_{i}$ to 1 as follows. By the Divisor Equation (see eg Abramovich-Graber-Vistoli [2, Theorem 8.3.1]) and Equation (5), the big quantum product becomes

$$
\left(\phi_{\alpha} \bullet_{\tau} \phi_{\beta}, \phi_{\gamma}\right)=\sum_{d \in \operatorname{Eff}(\mathcal{X})} \sum_{n \geq 0} \frac{Q^{d} e^{\int_{d} \sigma}}{n !}\left\langle\phi_{\alpha}, \phi_{\beta}, \phi_{\gamma}, \tau^{\prime}, \ldots, \tau^{\prime}\right\rangle_{0, d, n+3}^{\mathcal{X}}
$$

where $\tau=\sigma+\tau^{\prime}$ is the decomposition of $\tau$ into the nontwisted second cohomology class $\sigma$ and the sum $\tau^{\prime}$ of other components. Our convergence assumption therefore implies that the specialization $\left.\bullet_{\tau}\right|_{Q_{i}=1}$ is analytic on a domain $U \subset H_{\text {orb }}^{\bullet}(\mathcal{X} ; \mathbb{C})$ of the form:

$$
U=\left\{\tau=\left(\sigma, \tau^{\prime}\right): \mathfrak{i}\left(\int_{d} \sigma\right)<-M \text { for all } d \in \operatorname{Eff}(\mathcal{X}) \backslash\{0\},\left\|\tau^{\prime}\right\|<\epsilon\right\}
$$

for a sufficiently big $M>0$ and a sufficiently small $\epsilon>0$. Note that $\bullet_{\tau}$ for sufficiently small values of $Q$ equals $\bullet{ }_{\tau} \mid Q_{i}=1$ with $\hat{\tau}=\tau+\sum_{i} p_{i} \log Q_{i}$. In particular, the origin $\tau=Q=0$ of $H$ corresponds to the limit direction $\Re\left(\int_{d} \sigma\right) \rightarrow-\infty, \tau^{\prime} \rightarrow 0$ in $U$. This is called the large radius limit. The specialization $\left.\mathcal{E}_{\mathrm{A}}^{\mathrm{big}}\right|_{Q_{i}=1}$ is defined as an analytic VSHS on the base $U$ in the same way as in Section 2.3.1. Because the map $\mathbb{J}$ is a solution to the differential equation (20), the specialization

$$
\left.\mathbb{J}\right|_{Q_{i}=1}:\left.\mathcal{E}_{\mathrm{A}}^{\mathrm{big}}\right|_{Q_{i}=1} \longrightarrow H_{\mathrm{orb}}^{\bullet}(\mathcal{X} ; \mathbb{C}) \otimes \mathcal{O}_{U}\left\{z, z^{-1}\right\}
$$

is well-defined on $U$ and gives an analytic family of moving subspaces:

$$
\mathbb{E}_{\tau}:=\left.\left.\mathbb{J}\right|_{Q_{i}=1}\left(\left.\mathcal{E}_{\mathrm{A}, \tau}^{\mathrm{big}}\right|_{Q_{i}=1}\right) \subset \mathcal{H}_{\mathcal{X}}\right|_{Q_{i}=1}:=H_{\mathrm{orb}}^{\bullet}(\mathcal{X} ; \mathbb{C}) \otimes \mathbb{C}\left\{z, z^{-1}\right\}
$$


In view of the relations (19) and (21), we define the analytic version of Givental's cone to be the set

$$
\mathcal{L}_{\mathcal{X}}^{\mathrm{an}}:=\left.\bigcup_{\tau \in U} z \mathbb{E}_{\tau}\right|_{z \mapsto-z}
$$

Note that the analytic version is no longer a germ at some point. In fact if we work with an $L^{2}$ version of the symplectic formalism, replacing $\left.\mathcal{H}_{\mathcal{X}}\right|_{Q_{i}=1}$ with $H_{\text {orb }}^{\bullet}(\mathcal{X} ; \mathbb{C}) \otimes$ $L^{2}\left(S^{1}, \mathbb{C}\right)$ then, in a neighbourhood of the section $\left.\tau \mapsto J_{\mathcal{X}}(\tau,-z)\right|_{Q_{i}=1}, \mathcal{L}_{\mathcal{X}}^{\text {an }}$ has the structure of a Hilbert submanifold of $H_{\text {orb }}^{\bullet}(\mathcal{X} ; \mathbb{C}) \otimes L^{2}\left(S^{1}, \mathbb{C}\right)$ and this submanifold is the graph of the differential of the analytic function $\left.\mathcal{F}_{\mathcal{X}}^{0}\right|_{Q_{i}=1}$. When the quantum product $\left.\bullet_{\tau}\right|_{Q_{i}=1}$ is analytically continued to a bigger domain, the analytic Givental's cone $\mathcal{L}_{\mathcal{X}}^{\text {an }}$ can be enlarged to a bigger submanifold using the construction above. This is the analytic continuation of Givental's cone appearing in Conjecture 1.3.

\subsection{Small quantum cohomology}

Small quantum orbifold cohomology is a family of algebra structures on $H_{\text {orb }}^{\bullet}(\mathcal{X} ; \mathbb{C})$ defined, roughly speaking, by restricting the parameter $\tau$ of the big quantum product $\bullet_{\tau}$ to lie in $H^{2}(\mathcal{X} ; \mathbb{C}) \subset H_{\text {orb }}^{\bullet}(\mathcal{X} ; \mathbb{C})$. In this section we make this precise, and also explain how small quantum orbifold cohomology arises from a nonminiversal VSHS- the small A-model VSHS.

2.4.1 Small quantum orbifold cohomology From the Equation (24), the big quantum product $\bullet_{\tau}$ at a nontwisted second cohomology class $\tau \in H^{2}(\mathcal{X} ; \mathbb{C})$ becomes:

$$
\left(\alpha \bullet_{\tau} \beta, \gamma\right)_{\mathrm{orb}}=\sum_{d \in \operatorname{Eff}(\mathcal{X})} Q^{d} e^{\int_{d} \tau}\langle\alpha, \beta, \gamma\rangle_{0,3, d}^{\mathcal{X}}
$$

This shows that the Novikov parameters keep track of the modes of Fourier expansion in $\tau \in H^{2}(\mathcal{X}, \mathbb{C})$ and that the product $\bullet_{\tau}$ depends on $\tau$ only through the exponentiated Kähler parameters $e^{\tau^{i}}$. The small quantum orbifold cohomology of $\mathcal{X}$ is the possibly multivalued family of algebras $\left(H_{\text {orb }}^{\bullet}(\mathcal{X} ; \mathbb{C}), \circ_{q}\right)$ defined by

$$
\left(\alpha \circ_{q} \beta, \gamma\right)_{\text {orb }}=\sum_{d \in \operatorname{Eff}(\mathcal{X})} q^{d}\langle\alpha, \beta, \gamma\rangle_{0,3, d}^{\mathcal{X}}
$$

where the parameter $q$ lies on the torus

$$
\Upsilon=H^{2}(\mathcal{X} ; \mathbb{C}) / 2 \pi i H^{2}(\mathcal{X} ; \mathbb{Z})
$$

and $q^{d}$ denotes the following possibly multivalued function on $\Upsilon$ :

$$
q^{d}: \Upsilon \ni[\tau] \longmapsto \exp \left(\int_{d} \tau\right) \in \mathbb{C}^{\times} \quad \tau \in H^{2}(\mathcal{X} ; \mathbb{C})
$$


The cohomology groups here denote sheaf cohomology of the stack $\mathcal{X}$ and not of the coarse moduli space.

In order to make geometric sense of (27), we introduce coordinates on the torus $\Upsilon$ and consider the associated partial compactification. Let $p_{1}, \ldots, p_{r}$ be an integral basis of the free part of $H^{2}(\mathcal{X}, \mathbb{Z})$ such that $p_{i}$ evaluates nonnegatively on $\operatorname{Eff}(\mathcal{X})$. We define $\mathbb{C}^{\times}$-valued coordinates $q_{i}$ on $\Upsilon$ by

$$
q_{i}: \Upsilon \ni\left[\tau^{1} p_{1}+\cdots+\tau^{r} p_{r}\right] \longmapsto e^{\tau^{i}} \in \mathbb{C}^{\times}
$$

and for $d \in \operatorname{Eff}(\mathcal{X})$ we write:

$$
q^{d}=q_{1}^{\int_{d} p_{1}} \cdots q_{r}^{\int_{d} p_{r}}
$$

where each $\int_{d} p_{i}$ is a nonnegative rational number. The coordinates $\left(q_{1}, \ldots, q_{r}\right)$ give a partial compactification of $\Upsilon, \Upsilon \hookrightarrow \mathbb{C}^{r}$, and the small quantum product $\circ_{q}$ defines a possibly multivalued family of associative algebras in a formal neighbourhood of the origin in $\mathbb{C}^{r}$. The origin of $\mathbb{C}^{r}$ is called the large radius limit point of $\Upsilon$.

Remark 2.15 When pulled back to a suitable finite cover $\widetilde{\mathbb{C}^{r}}$ of $\mathbb{C}^{r}$, the product ${ }^{\circ} q$ becomes single-valued. The multivaluedness of the product $\circ_{q}$ happens only for orbifolds and introduces an orbifold singularity at the large radius limit point.

In our examples we already chose suitable integral bases $\left\{p_{i}\right\}$ for $H^{2}(\mathcal{X}, \mathbb{Z})$ in Section 2.1.1. This gives coordinates on $\Upsilon$, which we denote by $q_{1}, q_{2}$ if $\mathcal{X}=\mathbb{F}_{2}$ or $\mathbb{F}_{3}$ and by $q$ if $\mathcal{X}=\mathbb{P}(1,1,2)$ or $\mathbb{P}(1,1,1,3)$. In many cases, including the examples in our paper, the small quantum product $\circ_{q}$ is known to be convergent in a neighbourhood of the large radius limit point. In what follows we will assume this, writing $U_{\mathrm{A}} \subset \mathbb{C}^{r}$ for the domain of convergence of $\circ_{q}$ and $\mathcal{M}_{\mathrm{A}}=U_{\mathrm{A}} \cap \Upsilon . \mathcal{M}_{\mathrm{A}}$ is called the Kähler moduli space or A-model moduli space.

Remark 2.16 The Novikov variables $Q$ and $Q_{1}, Q_{2}$ are not the same as the parameters $q$ and $q_{1}, q_{2}$ for small quantum cohomology. But the restriction of the big quantum product $\bullet_{\tau}$ to the locus $\tau \in H^{2}(\mathcal{X} ; \mathbb{C})$ can be recovered from the small quantum product $\circ_{q}$ by setting

$$
q=Q e^{t}
$$

where $\tau=t p$

$$
\text { and } \mathcal{X}=\mathbb{P}(1,1,2) \text { or } \mathbb{P}(1,1,1,3)
$$

or

$$
q_{1}=Q_{1} e^{\tau^{1}}, q_{2}=Q_{2} e^{\tau^{2}}
$$

where $\tau=\tau^{1} p_{1}+\tau^{2} p_{2}$

and $\mathcal{X}=\mathbb{F}_{2}$ or $\mathbb{F}_{3}$. 
2.4.2 The small A-model VSHS The small A-model VSHS has base $\mathcal{M}_{\mathrm{A}}$. It is the free $\mathcal{O}_{\mathcal{M}_{\mathrm{A}}}\{z\}$-module

$$
\mathcal{E}_{\mathrm{A}}=H_{\text {orb }}^{\bullet}(\mathcal{X} ; \mathbb{C}) \otimes \mathcal{O}_{\mathcal{M}_{\mathrm{A}}}\{z\}
$$

with flat $z$-connection given by

$$
\nabla^{z}=z d+\sum_{i=1}^{r}\left(p_{i} \circ_{q}\right) \frac{d q_{i}}{q_{i}}
$$

pairing given by

$$
\begin{aligned}
\mathcal{E}_{\mathrm{A}} \times \mathcal{E}_{\mathrm{A}} & \longrightarrow \mathcal{O}_{\mathcal{M}_{\mathrm{A}}}\{z\} \\
f(z) \times g(z) & \longmapsto(f(-z), g(z))_{\mathrm{orb}}
\end{aligned}
$$

and grading operator Gr: $\mathcal{E}_{\mathrm{A}} \rightarrow \mathcal{E}_{\mathrm{A}}$ and Euler vector field $E$ given by

$$
\begin{aligned}
\mathrm{Gr} & =2 z \partial_{z}+2 d_{E}+\mathrm{Gr}_{0} \\
E & =\sum_{i=1}^{r} \rho_{i} q_{i} \frac{\partial}{\partial q_{i}}
\end{aligned}
$$

where $\mathrm{Gr}_{0}$ is the usual grading operator on orbifold cohomology and $c_{1}(X)=\sum_{i} \rho_{i} p_{i}$. The Dubrovin connection $\nabla^{z}$ here is independent of our choice of coordinates on $\mathcal{M}_{\mathrm{A}}$; it extends to a connection on $U_{\mathrm{A}}$ with a logarithmic singularity along the normal crossing divisor $q_{1} q_{2} \cdots q_{r}=0$ (Deligne's extension).

The flat $z$-connection $\nabla^{z}$ makes $\mathcal{E}_{\mathrm{A}}$ into a $D$-module in the sense of Givental [25]. When we want to emphasize this structure, we will refer to $\mathcal{E}_{\mathrm{A}}$ as the quantum $D_{-}$ module. See Guest [29] and Iritani [39] for more on this.

One obtains the small A-model VSHS from the big A-model VSHS by restricting $\tau$ to lie in $H^{2}(\mathcal{X}, \mathbb{C})$ and specializing Novikov variables $Q_{i}$ to 1 . In the moving subspace realization, the small VSHS therefore corresponds a subfamily of tangent spaces to Givental's Lagrangian cone $\mathcal{L}_{\mathcal{X}}^{\text {an }}$, and to the following subcone of $\mathcal{L}_{\mathcal{X}}^{\text {an }}$ :

$$
\left.\bigcup_{\tau \in H^{2}(\mathcal{X} ; \mathbb{C})} z \mathbb{E}_{\tau}^{\mathcal{X}}\right|_{z \mapsto-z} .
$$

This "small subcone" has a standard slice, the small $J$-function, which is obtained from the big $J$-function by restricting $\tau$ to lie in $H^{2}(\mathcal{X} ; \mathbb{C})$ and then setting the Novikov variables to 1 . In our examples we find, by applying the Divisor Equation to 
(18), that the small $J$-functions of $\mathbb{P}(1,1,1,3), \mathbb{F}_{3}, \mathbb{P}(1,1,2)$ and $\mathbb{F}_{2}$ are:

$$
\begin{aligned}
& J_{\mathbb{P}(1,1,2)}(q, z)=z q^{p / z}\left(\mathbf{1}_{0}+\sum_{\substack{d: 2 d \in \mathbb{Z} \\
d>0}} \sum_{\alpha=1}^{N} q^{d}\left\langle\frac{\phi^{\alpha}}{z(z-\psi)}\right\rangle_{0,1, d}^{\mathbb{P}(1,1,2)} \phi_{\alpha}\right) \\
& J_{\mathbb{F}_{2}}\left(q_{1}, q_{2}, z\right)=z q_{1}^{p_{1} / z} q_{2}^{p_{2} / z}\left(1+\sum_{\substack{k, l \geq 0 \\
k, l) \neq(0,0)}} \sum_{\alpha=1}^{N} q_{1}^{k} q_{2}^{l}\left\langle\frac{\phi^{\alpha}}{z(z-\psi)}\right\rangle_{0,1,(k, l)}^{\mathbb{F}_{2}} \phi_{\alpha}\right)
\end{aligned}
$$

$$
\begin{aligned}
J_{\mathbb{P}(1,1,1,3)}(q, z) & =z q^{p / z}\left(\mathbf{1}_{0}+\sum_{\substack{d: 3 d \in \mathbb{Z} \\
d>0}} \sum_{\alpha=1}^{N} q^{d}\left\langle\frac{\phi^{\alpha}}{z(z-\psi)}\right)_{0,1, d}^{\mathbb{P}(1,1,1,3)} \phi_{\alpha}\right) \\
J_{\mathbb{F}_{3}}\left(q_{1}, q_{2}, z\right) & =z q_{1}^{p_{1} / z} q_{2}^{p_{2} / z}\left(1+\sum_{\substack{k, l \geq 0 \\
k, l) \neq(0,0)}} \sum_{\alpha=1}^{N} q_{1}^{k} q_{2}^{l}\left\langle\frac{\phi^{\alpha}}{z(z-\psi)}\right\rangle_{0,1,(k, l)}^{\mathbb{F}_{3}} \phi_{\alpha}\right)
\end{aligned}
$$

These are multivalued analytic functions $\mathcal{M}_{\mathrm{A}} \rightarrow H_{\text {orb }}^{\bullet}(\mathcal{X} ; \mathbb{C}) \otimes \mathbb{C}\left\{z, z^{-1}\right\}$, for the appropriate choice of target space $\mathcal{X}$.

The small $J$-function corresponds to the unit section of $\mathcal{E}_{\mathrm{A}}$, ie $z^{-1} J_{\mathcal{X}}(q, z)=\iota_{q}(1)$. Since in the cases at hand the small quantum orbifold cohomology algebra of $\mathcal{X}$ is generated by $H^{2}(\mathcal{X} ; \mathbb{C})$, the small A-model VSHS is generated by this section together with its derivatives. As discussed above Definition 2.8, this makes $\mathcal{E}_{\mathrm{A}} / z \mathcal{E}_{\mathrm{A}}$ into a Frobenius algebra: the algebra structure here is the small quantum orbifold cohomology of $\mathcal{X}$ and the pairing is the orbifold Poincare pairing.

\subsection{Mirror symmetry}

We now define the $B$-model VSHS discussed in the Introduction, and explain what we mean by mirror symmetry.

2.5.1 The B-model VSHS A Landau-Ginzburg model in this context is a holomorphic family $\pi: Z \rightarrow \mathcal{M}_{\mathrm{B}}$ of affine Calabi-Yau manifolds-for us they will be algebraic tori-together with a function $W: Z \rightarrow \mathbb{C}$ called the superpotential and a section $\omega$ of the relative canonical sheaf $K_{Z / \mathcal{M}_{\mathrm{B}}}$ which gives a holomorphic volume form $\omega_{y}$ on each fiber $Z_{y}=\pi^{-1}(y)$. The base space $\mathcal{M}_{\mathrm{B}}$ of the family is called the $B$-model moduli space. Landau-Ginzburg models which correspond under mirror symmetry to the quantum cohomology of toric varieties have been constructed by Givental [24; 26] and Hori-Vafa [34]. In this section we explain how to obtain a VSHS-the B-model VSHS-from a Landau-Ginzburg model. 
Assumptions 2.17 We can assume (by deleting any points at which this condition fails to hold) that for each $y_{0} \in \mathcal{M}_{\mathrm{B}}$ there exists a neighbourhood $U$ of $y_{0}$ and a constant $M>0$ such that for all $y \in U$, all the critical points of $W_{y}$ are contained in the set $\left\{x \in Z_{y}:\left|W_{y}(x)\right|<M\right\}$. We further assume that:

(a) The family of pairs

$$
\begin{array}{ll}
\left(Z_{y}, N_{y, \theta}\right) \quad \text { where } \quad & N_{y, \theta}=\left\{x: \Re\left(W_{y}(x) e^{\mathrm{i} \theta}\right) \leq-M\right\} \\
& \left(y, e^{\mathrm{i} \theta}\right) \in U \times S^{1}
\end{array}
$$

is topologically locally trivial on $U \times S^{1}$.

(b) All the data $\pi: Z \rightarrow \mathcal{M}_{\mathrm{B}}, W, \omega$ are algebraic.

(c) There is a complete Kähler metric on $Z_{y}$ such that the set

$$
\left\{x \in Z_{y}:\left\|\operatorname{grad} W_{y}(x)\right\| \leq C\right\}
$$

is compact for some $C>0$.

(d) The critical points of $W_{y}$ are isolated and generically nondegenerate.

These assumptions are satisfied by the Landau-Ginzburg mirrors to $\mathbb{P}(1,1,2), \mathbb{F}_{2}$, $\mathbb{P}(1,1,1,3)$, and $\mathbb{F}_{3}$; furthermore Iritani [36, Section 3.2] showed that they hold for the mirror to a general compact weak Fano toric orbifold. We will use condition (a) when constructing a local system of relative homology groups. Condition (b) is much stronger than we need: we use it only to ensure the convergence of certain integrals, and this certainly follows from a polynomial-growth condition on the integrand (35). The remaining conditions allow us to use Morse theory. Assumption (c) implies that we can choose a metric without introducing critical points "at infinity": it holds for the mirrors to toric varieties. In the examples at hand, the critical points of $W_{y}$ are always distinct and nondegenerate: we denote them by $\sigma_{1}, \ldots, \sigma_{N}$.

Under our assumptions, a Landau-Ginzburg model determines a local system $R^{\vee}$ on $\mathcal{M}_{\mathrm{B}} \times \mathbb{C}^{\times}$with fiber over $(y, z)$ equal to the relative homology group

$$
R_{(y, z)}^{\vee}=H_{n}\left(Z_{y},\left\{x \in Z_{y}: \Re\left(W_{y}(x) / z\right) \ll 0\right\}\right) .
$$

Let $\mathcal{O}_{\mathcal{M}_{\mathrm{B}} \times \mathbb{C}^{\times}}$denote the analytic structure sheaf. The associated locally free sheaf $\mathcal{R}^{\vee}=R^{\vee} \otimes \mathcal{O}_{\mathcal{M}_{\mathrm{B}} \times \mathbb{C}^{\times}}$has a Gauss-Manin connection, which is flat. We construct flat sections of $\mathcal{R}^{\vee}$ using Morse theory, defining the cycle $\Gamma_{k}(y, z), k \in\{1, \ldots, N\}$, to be the closure of the union of downward gradient flowlines for the function $x \mapsto$ $\Re\left(W_{y}(x) / z\right)$ from the critical point $\sigma_{k}$ of $W_{y}$. If the imaginary parts of the critical values of $W_{y} / z$ are all distinct then the image of $\Gamma_{k}(y, z)$ under $W_{y} / z$ becomes 
a negative half-line from $W_{y}\left(\sigma_{k}\right) / z$ parallel to the real axis. The cycles $\Gamma_{k}(y, z)$ form a basis for the relative homology group $R_{(y, z)}^{\vee}$. Note that these sections become multivalued under analytic continuation: $\Gamma_{k}(y, z)$ has monodromy in both $y$ and $z$.

The dual bundle $\mathcal{R}$ on $\mathcal{M}_{\mathrm{B}} \times \mathbb{C}^{\times}$has fiber over $(y, z)$ equal to the relative cohomology group $H^{n}\left(Z_{y},\left\{x \in Z_{y}: \Re\left(W_{y}(x) / z\right) \ll 0\right\}\right)$. This bundle also has a flat Gauss-Manin connection, as well as a distinguished section

$$
(y, z) \longmapsto \exp \left(W_{y} / z\right) \omega_{y} .
$$

The coordinates of this section with respect to the flat frame dual to $\Gamma_{1}(y, z), \ldots$, $\Gamma_{N}(y, z)$ are oscillating integrals:

$$
\mathcal{I}_{k}(y, z)=\int_{\Gamma_{k}(y, z)} \exp \left(W_{y} / z\right) \omega_{y}
$$

We will consider only sections of $\mathcal{R}$ represented by differential forms

$$
f(x, z) \exp (W(x) / z) \omega_{y}, \quad f(x, z) \in(\pi \times \mathrm{id})_{\star} \mathcal{O}_{Z \times \mathbb{C}^{\times}}
$$

such that $x \mapsto f(x, z)$ is algebraic on each fiber $Z_{y}$; note that integrals of such forms over cycles $\Gamma_{k}(y, z)$ are convergent.

Definition 2.18 Given a Landau-Ginzburg model $\left(\pi: Z \rightarrow \mathcal{M}_{\mathrm{B}}, W, \omega\right)$, we define the associated $B$-model VSHS as follows. The base of the B-model VSHS is $\mathcal{M}_{\mathrm{B}}$. Let $\mathcal{E}_{\mathrm{B}}$ be the $\mathcal{O}_{\mathcal{M}_{\mathrm{B}}}\{z\}$-module consisting of sections of $\mathcal{R}$ of the form (35) with $f$ regular in a small neighbourhood of $z=0$. Let $\nabla^{z}$ be the flat $z$-connection on $\mathcal{E}_{\mathrm{B}}$ given by

$$
\nabla_{X}^{z}=z \nabla_{X}^{\mathrm{GM}}
$$

where $X$ on the left-hand side is a vector field on $\mathcal{M}_{\mathrm{B}}, \nabla^{\mathrm{GM}}$ is the Gauss-Manin connection on $\mathcal{R}$, and $X$ on the right-hand side denotes the standard lift to a vector field on $\mathcal{M}_{\mathrm{B}} \times \mathbb{C}^{\times}$. The pairing on $\mathcal{E}_{\mathrm{B}}$ is defined as the dual to the intersection pairing on relative homology groups:

$$
R_{(y,-z)}^{\vee} \otimes R_{(y, z)}^{\vee} \rightarrow \mathbb{C}
$$

As in Section 2.4.2, the flat $z$-connection $\nabla^{z}$ makes $\mathcal{E}_{\mathrm{B}}$ into a $D$-module in the sense of Givental [25]. When we want to emphasize this structure, we will refer to $\mathcal{E}_{\mathrm{B}}$ as the mirror D-module.

The pairing on $\mathcal{E}_{\mathrm{B}}$ is given, at generic $y$, by

$$
\left(\left[s_{1}\right],\left[s_{2}\right]\right)_{\mathcal{E}_{\mathrm{B}}}=\frac{1}{(2 \pi \mathrm{i} z)^{n}} \sum_{k=1}^{N} \int_{\Gamma_{k}(y,-z)} s_{1}(-z) \cdot \int_{\Gamma_{k}(y, z)} s_{2}(z)
$$


because $\left\{\Gamma_{k}(y,-z)\right\}_{k}$ and $\left\{\Gamma_{k}(y, z)\right\}_{k}$ are mutually-dual bases for the relative homology groups. We now check that the data in Definition 2.18 satisfy the axioms for a VSHS.

Lemma 2.19 The pairing $(\cdot, \cdot)$ takes values in $\mathcal{O}_{\mathcal{M}_{\mathrm{B}}}\{z\}$. Also, $\mathcal{E}_{\mathrm{B}}$ is a free $\mathcal{O}_{\mathcal{M}_{\mathrm{B}}}\{z\}-$ module of the same rank as $\mathcal{R}$.

Proof The method of stationary phase gives the asymptotic expansion of the oscillating integrals:

$$
\int_{\Gamma_{i}(y, z)} f(x, z) \exp (W(x) / z) \omega_{y} \sim \frac{(-2 \pi z)^{n / 2}}{\sqrt{\operatorname{Hess} W_{y}\left(\sigma_{i}\right)}} e^{W_{y}\left(\sigma_{i}\right) / z}\left(f\left(\sigma_{i}, 0\right)+O(z)\right)
$$

for $f$ regular at $z=0$. Here $z$ goes to zero in an angular sector where the order of $\Im\left(W_{y}\left(\sigma_{1}\right) / z\right), \ldots, \Im\left(W_{y}\left(\sigma_{N}\right) / z\right)$ is unchanged. The Hessian of $W_{y}$ at $\sigma_{i}$ is calculated in terms of local coordinates $x^{1}, \ldots, x^{n}$ on $Z_{y}$ such that $\omega_{y}=d x^{1} \wedge \cdots \wedge d x^{n}$ near $\sigma_{i}$. At generic $y$, therefore, we have:

$$
\left(\left[f(x, z) e^{W / z} \omega_{y}\right],\left[g(x, z) e^{W / z} \omega_{y}\right]\right)_{\mathcal{E}_{\mathrm{B}}} \sim \sum_{k=1}^{N} \frac{f\left(\sigma_{k}, 0\right) g\left(\sigma_{k}, 0\right)}{\operatorname{Hess} W_{y}\left(\sigma_{k}\right)}+O(z)
$$

The first term on the right hand side here is the residue pairing of the elements $f(x, 0)$ and $g(x, 0)$ of the Jacobi ring of $W_{y}$. The left hand side is holomorphic on $0<|z|<\epsilon$ for some $\epsilon$; the above asymptotics imply that it is actually regular at $z=0$.

In a neighbourhood of each point $y \in \mathcal{M}_{\mathrm{B}}$, we can find fiberwise-algebraic functions $\phi_{k}(x) \in \pi_{\star} \mathcal{O}_{Z}, k=1, \ldots, N$, such that $\left[\phi_{k}(x)\right]$ forms a basis of the Jacobi ring $J\left(W_{y}\right)$. If $y$ is generic then we can choose $\phi_{k}$ such that $\phi_{i}\left(\sigma_{j}\right)=\delta_{i j}$. Let $s_{i}=$ $\left[\phi_{i}(x) e^{W / z} \omega_{y}\right]$ be the corresponding section of $\mathcal{E}_{\mathrm{B}}$. Then the Gram matrix $\left(s_{i}, s_{j}\right)_{\mathcal{E}_{\mathrm{B}}}$ is of the form $\left(\phi_{i}, \phi_{j}\right)_{W}+O(z)$ with the first term given by the residue pairing. The nondegeneracy of the matrix $\left(\phi_{i}, \phi_{j}\right)_{W}$ implies that $s_{1}, \ldots, s_{N}$ form an $\mathcal{O}_{\mathcal{M}_{\mathrm{B}}}\{z\}-$ basis of $\mathcal{E}_{\mathrm{B}}$.

In our examples it turns out that $\mathcal{E}_{\mathrm{B}}$ is generated by the single section (33) together with its derivatives. In other words

$$
\mathcal{E}_{\mathrm{B}} \cong \mathcal{O}_{\mathcal{M}_{\mathrm{B}}}\{z\}\left\langle z \mathcal{T}_{\mathcal{M}_{\mathrm{B}}}\right\rangle /\left\{P(y, z \partial, z): P \mathcal{I}_{k}(y, z)=0 \text { for all } k\right\}
$$

and so $\mathcal{E}_{\mathrm{B}}$ is generated as a $D$-module by oscillating integrals. The family of Frobenius algebras $\mathcal{E}_{\mathrm{B}} / z \mathcal{E}_{\mathrm{B}}$ determined by $\mathcal{E}_{\mathrm{B}}$ and the section (33) consists of the family of Jacobi rings $\bigcup_{y \in \mathcal{M}_{\mathrm{B}}} J\left(W_{y}\right)$ equipped with the residue pairing. 
Remark 2.20 In the Introduction we described the B-model VSHS as a family of subspaces $\mathbb{E}_{q}, q \in \mathcal{M}_{\mathrm{B}}$, in the fibers of a vector bundle $V \rightarrow \mathcal{M}_{\mathrm{B}}$ with flat connection. The vector bundle $V$ is $\mathcal{E}_{\mathrm{B}} \otimes_{\mathcal{O}_{\mathcal{M}_{\mathrm{B}}}\{z\}} \mathcal{O}_{\mathcal{M}_{\mathrm{B}}}\left\{z, z^{-1}\right\}$, the family of subspaces is the subbundle $\mathcal{E}_{\mathrm{B}}$ of $V$, and the connection on $V$ is $\nabla^{\mathrm{GM}}$.

2.5.2 The mirror conjecture and mirror theorems We now formulate a mathematical version of mirror symmetry, in the spirit of Givental's ICM lecture [24].

Conjecture 2.21 Let $\mathcal{E}_{\mathrm{A}}$ be the small A-model VSHS of $\mathcal{X}$; recall that this has base the A-model moduli space $\mathcal{M}_{\mathrm{A}}$. Let $\mathcal{E}_{\mathrm{B}}$ be the $B$-model VSHS associated to the Landau-Ginzburg mirror to $\mathcal{X}$; this has base the $B$-model moduli space $\mathcal{M}_{\mathrm{B}}$. Let $\Delta_{\epsilon}^{*} \subset \mathbb{C}$ be a punctured disc of radius $\epsilon$. There is an open set $U_{\mathrm{B}} \subset \mathcal{M}_{\mathrm{B}}$ with coordinates $\left(y_{1}, \ldots, y_{r}\right): U_{\mathrm{B}} \cong\left(\Delta_{\epsilon}^{*}\right)^{r}$ and a map mir: $U_{\mathrm{B}} \rightarrow \mathcal{M}_{\mathrm{A}}$ of the form

$$
\begin{array}{cc}
\left(y_{1}, \ldots, y_{r}\right) \longmapsto\left(q_{1}, \ldots, q_{r}\right) \quad \text { where } \quad & q_{i}=y_{i} \exp \left(f_{i}\left(y_{1}, \ldots, y_{r}\right)\right) \\
& f_{i}(0, \ldots, 0)=0
\end{array}
$$

such that there is an isomorphism of VSHSs:

$$
\left(\mathcal{E}_{\mathrm{B}}, \nabla^{z},(\cdot, \cdot)\right) \cong \operatorname{mir}^{\star}\left(\mathcal{E}_{\mathrm{A}}, \nabla^{z},(\cdot, \cdot)\right)
$$

In the graded case this isomorphism also preserves the operator Gr.

The map mir here is called the mirror map. We have seen that the VSHSs $\mathcal{E}_{\mathrm{A}}$ and $\mathcal{E}_{\mathrm{B}}$ give rise to families of Frobenius algebras: $\mathcal{E}_{\mathrm{A}} / z \mathcal{E}_{\mathrm{A}}$ gives the small quantum orbifold cohomology algebra of $\mathcal{X}$ equipped with the orbifold Poincaré pairing, and $\mathcal{E}_{\mathrm{B}} / z \mathcal{E}_{\mathrm{B}}$ gives the family of Jacobi rings $\bigcup_{y \in \mathcal{M}_{\mathrm{B}}} J\left(W_{y}\right)$ equipped with the residue pairing. Suppose in addition that the unit section 1 of $\mathcal{E}_{\mathrm{A}}$ corresponds under (37) to the distinguished section (33) of $\mathcal{E}_{\mathrm{B}}$; this condition holds in the examples at hand. Then Conjecture 2.21 implies that there is a grading-preserving linear isomorphism

$$
\operatorname{Mir}_{y}: J\left(W_{y}\right) \rightarrow H_{\text {orb }}^{\bullet}(\mathcal{X} ; \mathbb{C})
$$

which matches the product on the Jacobi ring $J\left(W_{y}\right)$ with the small quantum product ${ }^{\circ} \operatorname{mir}(y)$ and matches the orbifold Poincaré pairing with the residue pairing.

How to prove Conjecture 2.21 In the examples that we consider below, Conjecture 2.21 is simply a reformulation of mirror theorems proved by Givental [26] (or equivalently by Lian-Liu-Yau [41]) and by Coates-Corti-Lee-Tseng [15]. But this reformulation is essential to our argument in Sections 3 and 4: it allows us to give a systematic construction of flat structures near the cusps of the B-model moduli space 
$\mathcal{M}_{\mathrm{B}}$, and to compare the flat structures associated to different cusps. We proceed as follows.

The oscillating integrals (34) form a basis of solutions ${ }^{11}$ to the mirror $D$-module $\mathcal{E}_{\mathrm{B}}$. These solutions are multivalued in both $y$ and $z$. The system of differential equations in $y$ satisfied by the oscillating integrals is called the Picard-Fuchs system-see equations (38), (40), (61), (63). Another basis of solutions to the Picard-Fuchs system is given by the components of a cohomology-valued hypergeometric function called the $I-$ function $I_{\mathcal{X}}(y, z)$-see equations (39), (41), (62), (64). Thus the mirror $D$-module $\mathcal{E}_{\mathrm{B}}$ is isomorphic to the $D$-module generated by the $I$-function. The $I$-functions are multivalued in $y$ but single-valued in $z$.

On the other hand, in our examples the small quantum orbifold cohomology algebra is generated by $H^{2}(\mathcal{X} ; \mathbb{C})$ and so the A-model VSHS $\mathcal{E}_{\mathrm{A}}$ is generated by the small $J$-function $J_{\mathcal{X}}(q, z)$ together with its derivatives. This implies that $\mathcal{E}_{\mathrm{A}}$ is generated as a $D$-module by the small $J$-function. The small $J$-functions are multivalued in $q$ but single-valued in $z$-see equations (29), (30), (31), (32).

A Givental-style mirror theorem states that the I-function and the small $J$-function coincide after a suitable change of variables $y \mapsto q(y)$ :

$$
J_{\mathcal{X}}(q(y), z)=I_{\mathcal{X}}(y, z)
$$

The change of variables $y \mapsto q(y)$ here gives the mirror map in Conjecture 2.21. Such a mirror theorem implies Conjecture 2.21, as we can then define the isomorphism (37) to be the $D$-module isomorphism which maps:

$$
\left(\mathcal{E}_{\mathrm{B}}\right)_{y} \ni\left[\exp \left(W_{y} / z\right) \omega_{y}\right] \quad \text { to } \quad z^{-1} I_{\mathcal{X}}(y, z)=z^{-1} J_{\mathcal{X}}(q, z) \in \iota_{q}\left(\left(\mathcal{E}_{\mathrm{A}}\right)_{q}\right)
$$

The matching of gradings and pairings under this isomorphism will be explained in Propositions 3.3 and 3.6 below.

A detailed proof of Conjecture 2.21 for a general compact weak Fano toric orbifold has been given by Iritani [36, Section 4.2].

\section{Example: $\mathbb{F}_{3}$ and $\mathbb{P}(1,1,1,3)$}

We now apply our general theory to the cases $\mathcal{X}=\mathbb{P}(1,1,1,3)$ and $Y=\mathbb{F}_{3}$. Following the prescriptions of Givental [26] and Hori-Vafa [34], we write down Landau-Ginzburg models which correspond under mirror symmetry to $\mathbb{F}_{3}$ and to $\mathbb{P}(1,1,1,3)$. Let $\mathcal{M}_{\mathbb{F}_{3}}$

\footnotetext{
${ }^{11}$ Recall that a solution to a $D$-module is a solution to the system of differential equations defining that $D$-module.
} 
denote the base of the Landau-Ginzburg mirror to $\mathbb{F}_{3}$ and let $\mathcal{M}_{\mathbb{P}(1,1,1,3)}$ denote the base of the Landau-Ginzburg mirror to $\mathbb{P}(1,1,1,3)$. We construct the B-model moduli space $\mathcal{M}_{\mathrm{B}}$, described in the Introduction, as a partial compactification of $\mathcal{M}_{\mathbb{F}_{3}}$. The space $\mathcal{M}_{\mathrm{B}}$ consists of a copy of $\mathcal{M}_{\mathbb{F}_{3}}$ together with a copy of $\mathcal{M}_{\mathbb{P}(1,1,1,3)}$ as a "divisor at infinity". We form a Landau-Ginzburg model with base $\mathcal{M}_{\mathrm{B}}$ by patching together the mirrors to $\mathbb{F}_{3}$ and $\mathbb{P}(1,1,1,3)$, and define the $\mathrm{B}$-model VSHS to be the VSHS associated to this Landau-Ginzburg model.

The space $\mathcal{M}_{\mathrm{B}}$ has two cusps, one of which corresponds to $\mathbb{F}_{3}$ and the other to $\mathbb{P}(1,1,1,3)$. We show that there is an opposite subspace canonically associated to each cusp (Theorem 3.5) and that these opposite subspaces give rise, in the sense described in the Introduction, to the small quantum cohomology of $\mathbb{F}_{3}$ and the small quantum orbifold cohomology of $\mathbb{P}(1,1,1,3)$. We show that these two opposite subspaces $d o$ not agree with each other under parallel transport in $\mathcal{E}_{\mathrm{B}}$-this amounts to computing the analytic continuation of the $I$-function $I_{Y}(y, z)$ mentioned in Section 2.5.2-and from this we prove Theorem 1.2 and Conjecture 1.3.

\subsection{The Landau-Ginzburg mirror to $\mathbb{F}_{3}$}

The Landau-Ginzburg mirror of $\mathbb{F}_{3}$ is a family of algebraic tori $\pi: Z \rightarrow \mathcal{M}_{\mathbb{F}_{3}}$ together with a superpotential $W: Z \rightarrow \mathbb{C}$ and a holomorphic volume form on each fiber of $\pi$. Recall that $\mathbb{F}_{3}$ is defined as a GIT quotient of $\mathbb{C}^{5}$ by $\left(\mathbb{C}^{\times}\right)^{2}$ where $\left(\mathbb{C}^{\times}\right)^{2}$ acts via the inclusion:

$$
\left(\mathbb{C}^{\times}\right)^{2} \hookrightarrow\left(\mathbb{C}^{\times}\right)^{5} \quad(s, t) \mapsto\left(s, s, s, s^{-3} t, t\right)
$$

The mirror family $\pi: Z \rightarrow \mathcal{M}_{\mathbb{F}_{3}}$ is given by restricting the dual of this inclusion

$$
\begin{aligned}
\pi:\left(\mathbb{C}^{\times}\right)^{5} & \longrightarrow\left(\mathbb{C}^{\times}\right)^{2} \\
\left(w_{1}, \ldots, w_{5}\right) & \longmapsto\left(w_{1} w_{2} w_{3} w_{4}^{-3}, w_{4} w_{5}\right)
\end{aligned}
$$

to the open subset $\mathcal{M}_{\mathbb{F}_{3}} \subset\left(\mathbb{C}^{\times}\right)^{2}$ defined by:

$$
\mathcal{M}_{\mathbb{F}_{3}}=\left\{\left(y_{1}, y_{2}\right) \in\left(\mathbb{C}^{\times}\right)^{2}: y_{1} \neq-\frac{1}{27}\right\}
$$

The superpotential $W$ is

$$
W=w_{1}+w_{2}+w_{3}+w_{4}+w_{5}
$$

and the holomorphic volume form $\omega_{y}$ on the fiber $Z_{y}=\pi^{-1}\left(y_{1}, y_{2}\right)$ is:

$$
\omega_{y}=\frac{d \log w_{1} \wedge \cdots \wedge d \log w_{5}}{d \log y_{1} \wedge d \log y_{2}}
$$


We deleted the locus $y_{1}=-\frac{1}{27}$ from $\mathcal{M}_{\mathbb{F}_{3}}$ to ensure that Assumptions 2.17 hold. It is straightforward to show that the oscillating integrals (34) satisfy the Picard-Fuchs equations:

$$
\begin{aligned}
D_{2}\left(D_{2}-3 D_{1}\right) f & =y_{2} f \\
D_{1}^{3} D_{2}\left(D_{2}-z\right)\left(D_{2}-2 z\right) f & =y_{1} y_{2}^{3} f \\
D_{1}^{3} D_{2}\left(D_{2}-z\right) f & =y_{1} y_{2}^{2}\left(D_{2}-3 D_{1}\right) f \\
D_{1}^{3} D_{2} f & =y_{1} y_{2}\left(D_{2}-3 D_{1}\right)\left(D_{2}-3 D_{1}-z\right) f \\
D_{1}^{3} f & =y_{1}\left(D_{2}-3 D_{1}\right)\left(D_{2}-3 D_{1}-z\right)\left(D_{2}-3 D_{1}-2 z\right) f
\end{aligned}
$$

where $D_{1}=z y_{1} \frac{\partial}{\partial y_{1}}$ and $D_{2}=z y_{2} \frac{\partial}{\partial y_{2}}$.

\subsection{Mirror symmetry for $\mathbb{F}_{3}$}

We apply Givental's mirror theorem for toric varieties [26, Theorem 0.1$]$. The $I-$ function

(39) $I_{\mathbb{F}_{3}}\left(y_{1}, y_{2}, z\right)$

$$
=z \sum_{k, l \geq 0} \frac{y_{1}^{k+p_{1} / z} y_{2}^{l+p_{2} / z} \prod_{m=-\infty}^{0}\left(p_{2}-3 p_{1}+m z\right)}{\prod_{m=1}^{k}\left(p_{1}+m z\right)^{3} \prod_{m=1}^{l}\left(p_{2}+m z\right) \prod_{m=-\infty}^{l-3 k}\left(p_{2}-3 p_{1}+m z\right)}
$$

where $p_{1}, p_{2}$ is the basis of $H^{2}\left(\mathbb{F}_{3}\right)$ defined in Section 2.1.1, also satisfies the PicardFuchs system (38). It coincides with the small $J$-function (32) after a change of variables $\left(y_{1}, y_{2}\right) \mapsto\left(q_{1}, q_{2}\right)$ :

$$
J_{\mathbb{F}_{3}}\left(q_{1}, q_{2}, z\right)=I_{\mathbb{F}_{3}}\left(y_{1}, y_{2}, z\right)
$$

As we explained in Section 2.5.2, this proves Conjecture 2.21 for $\mathbb{F}_{3}$.

Since $J_{\mathbb{F}_{3}}\left(q_{1}, q_{2}, z\right)=z+p_{1} \log q_{1}+p_{2} \log q_{2}+O\left(z^{-1}\right)$, we can read off the mirror map $\left(y_{1}, y_{2}\right) \mapsto\left(q_{1}, q_{2}\right)$ by expanding the $I$-function as a Laurent series in $z^{-1}$. This gives:

$$
\begin{aligned}
& q_{1}=y_{1} \exp \left(3 \sum_{k \geq 1}(-1)^{k} \frac{(3 k-1) !}{(k !)^{3}} y_{1}^{k}\right) \\
& q_{2}=y_{2} \exp \left(\sum_{k \geq 1}(-1)^{k+1} \frac{(3 k-1) !}{(k !)^{3}} y_{1}^{k}\right)
\end{aligned}
$$


and hence:

$$
\begin{aligned}
& y_{1}=q_{1}+6 q_{1}^{2}+9 q_{1}^{3}+56 q_{1}^{4}-300 q_{1}^{5}+\cdots \\
& y_{2}=q_{2}\left(1-2 q_{1}+5 q_{1}^{2}-32 q_{1}^{3}+286 q_{1}^{4}-3038 q_{1}^{5}-\cdots\right)
\end{aligned}
$$

The mirror map identifies a suitable neighbourhood ${ }^{12}$ of $y_{1}=y_{2}=0$ in $\mathcal{M}_{\mathbb{F}_{3}}$ with the Kähler moduli space $\mathcal{M}_{\mathrm{A}}$ of $\mathbb{F}_{3}$; here $\left(q_{1}, q_{2}\right)$ are coordinates on $\mathcal{M}_{\mathrm{A}}$. This identification matches up the Jacobi ring $J\left(W_{y}\right)$ of $W_{y}$ with the small quantum cohomology algebra of $\mathbb{F}_{3}$ at $\left(q_{1}, q_{2}\right)$ and the residue pairing with the Poincaré pairing.

\subsection{The Landau-Ginzburg mirror to $\mathbb{P}(1,1,1,3)$}

The mirror family $\pi$ is

$$
\begin{aligned}
\pi: Z=\left(\mathbb{C}^{\times}\right)^{4} & \longrightarrow \mathcal{M}_{\mathbb{P}(1,1,1,3)}=\left(\mathbb{C}^{\times}\right) \\
\left(w_{1}, w_{2}, w_{3}, w_{5}\right) & \longmapsto w_{1} w_{2} w_{3} w_{5}^{3}
\end{aligned}
$$

the superpotential $W$ is

$$
W=w_{1}+w_{2}+w_{3}+w_{5}
$$

and the holomorphic volume form $\omega_{y}$ on the fiber $Z_{y}=\pi^{-1}(y)$ is:

$$
\omega_{y}=\frac{d \log w_{1} \wedge d \log w_{2} \wedge d \log w_{3} \wedge d \log w_{5}}{d \log y}
$$

The nonstandard numbering of the coordinates here will be convenient later. The oscillating integrals (34) satisfy the Picard-Fuchs equation

$$
D^{3}(3 D)(3 D-z)(3 D-2 z) f=y f
$$

where $D=z y \frac{\partial}{\partial y}$.

\subsection{Mirror symmetry for $\mathbb{P}(1,1,1,3)$}

The mirror theorem we need here was proved by Coates-Corti-Lee-Tseng [15]. The $I-$ function

(41) $I_{\mathbb{P}(1,1,1,3)}(y, z)=z y^{p / z} \sum_{\substack{d: 3 d \in \mathbb{Z} \\ d \geq 0}} \frac{y^{d}}{\prod_{\substack{b:\langle b\rangle=\langle d\rangle \\ 0<b \leq d}}(p+b z)^{3} \prod_{\substack{b:\langle b\rangle=0 \\ 0<b \leq 3 d}}(3 p+b z)} \mathbf{1}_{\langle d\rangle}$

${ }^{12}$ This neighbourhood is $U_{\mathrm{B}}$ from Conjecture 2.21. 
also satisfies the Picard-Fuchs Equation (40). It coincides with the small $J$-function (31) after the (trivial) change of variables $q=y$ :

$$
J_{\mathbb{P}(1,1,1,3)}(q, z)=I_{\mathbb{P}(1,1,1,3)}(y, z)
$$

As discussed in Section 2.5.2, this proves Conjecture 2.21 for $\mathbb{P}(1,1,1,3)$. The moduli space $\mathcal{M}_{\mathbb{P}(1,1,1,3)}$ with coordinate $y$ is identified via the map $q=y$ with the Kähler moduli space $\mathcal{M}_{\mathrm{A}}$ of $\mathbb{P}(1,1,1,3)$; here $q$ is once again a coordinate on $\mathcal{M}_{\mathrm{A}}$. This identification matches the Jacobi ring $J\left(W_{q}\right)$ with the small quantum orbifold cohomology algebra of $\mathbb{P}(1,1,1,3)$ at $q$, and the residue pairing with the Poincare pairing.

\subsection{Constructing the B-model VSHS}

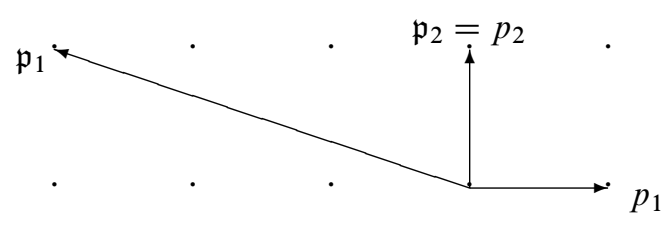

Figure 2: The secondary fan for $\mathbb{F}_{3}$

We now extend the Landau-Ginzburg mirror of $\mathbb{F}_{3}$ to a Landau-Ginzburg model with a larger base, defined in terms of the secondary fan for $\mathbb{F}_{3}$ (Figure 2). Take $w_{1}, w_{2}, w_{5}$ as coordinates on the fiber $Z_{y}$, so that:

$$
W_{y}=w_{1}+w_{2}+\frac{y_{1} y_{2}^{3}}{w_{1} w_{2} w_{5}^{3}}+\frac{y_{2}}{w_{5}}+w_{5}
$$

The toric orbifold $\overline{\mathcal{M}}$ associated to the secondary fan for $\mathbb{F}_{3}$ gives a compactification of $\mathcal{M}_{\mathbb{F}_{3}}$. One coordinate patch on $\overline{\mathcal{M}}$ comes from the Kähler cone of $\mathbb{F}_{3}$, which is the cone in the secondary fan spanned by $p_{1}=(1,0)$ and $p_{2}=(0,1)$. The vectors $p_{1}, p_{2}$ are dual to the coordinates $y_{1}, y_{2}$ on $\mathcal{M}_{\mathbb{F}_{3}}$. The adjacent cone, spanned by $\mathfrak{p}_{1}=(-3,1)$ and $\mathfrak{p}_{2}=(0,1)$, defines another coordinate patch on $\overline{\mathcal{M}}$ : let $\mathfrak{y}_{1}, \mathfrak{y}_{2}$ be the coordinates dual to $\mathfrak{p}_{1}, \mathfrak{p}_{2}$. The two coordinate systems are related by:

$$
\mathfrak{y}_{1}=y_{1}^{-1 / 3} \quad \mathfrak{y}_{2}=y_{1}^{1 / 3} y_{2}
$$

Note that $\mathfrak{y}_{1}, \mathfrak{y}_{2}$ are multivalued and so are not honest coordinates on $\overline{\mathcal{M}}$. One should think of $\overline{\mathcal{M}}$ as an orbifold and of $\mathfrak{y}_{1}, \mathfrak{y}_{2}$ as a uniformizing system ${ }^{13}$ near a $\mathbb{Z} / 3 \mathbb{Z}$

${ }^{13}$ This is the mirror partner of Remark 2.15. 
quotient singularity at $\left(\mathfrak{y}_{1}, \mathfrak{y}_{2}\right)=0$. In the coordinates $\left(\mathfrak{y}_{1}, \mathfrak{y}_{2}\right)$, we have:

$$
W_{y}=w_{1}+w_{2}+\frac{\mathfrak{y}_{2}^{3}}{w_{1} w_{2} w_{5}^{3}}+\frac{\mathfrak{y}_{1} \mathfrak{y}_{2}}{w_{5}}+w_{5}
$$

We can therefore extend the family of tori $\pi$ and the superpotential $W_{y}$ across the locus $\left\{\mathfrak{y}_{1}=0\right\}$, where we see the Landau-Ginzburg mirror of $\mathbb{P}(1,1,1,3)$ :

$$
W=w_{1}+w_{2}+w_{3}+w_{5}, \quad w_{1} w_{2} w_{3} w_{5}^{3}=\mathfrak{y}_{2}^{3} .
$$

The locus $\mathfrak{y}_{1}=0, \mathfrak{y}_{2} \neq 0$ in $\overline{\mathcal{M}}$ is identified with the base $\mathcal{M}_{\mathbb{P}(1,1,1,3)}$ of the LandauGinzburg mirror of $\mathbb{P}(1,1,1,3)$ via the map $y=\mathfrak{y}_{2}^{3}$.

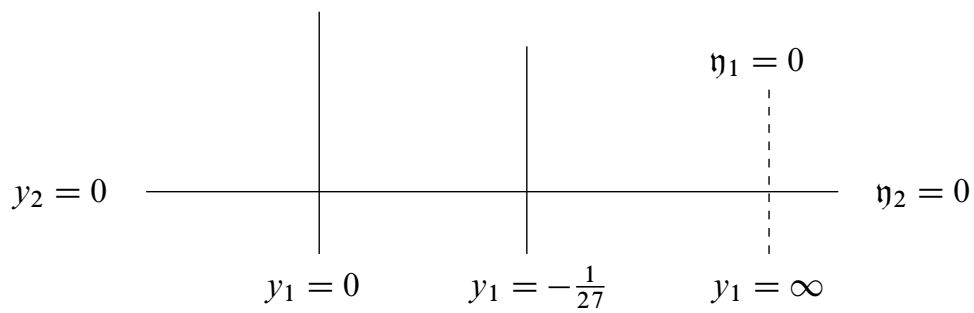

Figure 3: The B-model moduli space $\mathcal{M}_{\mathrm{B}}$

The base of our extended Landau-Ginzburg model, which we call the $B$-model moduli space $\mathcal{M}_{\mathrm{B}}$, is obtained from $\overline{\mathcal{M}}$ by deleting the closures of the loci $\left\{y_{1} y_{2}=0\right\}$ and $\left\{y_{1}=-1 / 27\right\}$. Equations (42) and (43) define a Landau-Ginzburg model over $\mathcal{M}_{\mathrm{B}}$ which contains the Landau-Ginzburg mirrors for $\mathbb{F}_{3}$ and for $\mathbb{P}(1,1,1,3)$ as subsets. The limit points $y_{1}=y_{2}=0$ and $\mathfrak{y}_{1}=\mathfrak{y}_{2}=0$ of $\mathcal{M}_{\mathrm{B}}$ are called the large radius limit points or cusps corresponding respectively to $\mathbb{F}_{3}$ and to $\mathbb{P}(1,1,1,3)$.

Let $\mathcal{E}$ denote the B-model VSHS with base $\mathcal{M}_{\mathrm{B}}$ defined by the Landau-Ginzburg model just described. (See Definition 2.18 for the B-model VSHS.) We equip $\mathcal{E}$ with the grading operator $\mathrm{Gr}: \mathcal{E} \rightarrow \mathcal{E}$ defined by

$$
\operatorname{Gr}\left[f(x, z) e^{W_{y} / z} \omega_{y}\right]=\left[\left(2 z \partial_{z}+2 \sum_{i=1}^{5} w_{i} \partial_{w_{i}}\right) f(x, z) e^{W_{y} / z} \omega_{y}\right]
$$

This satisfies the axioms for a graded VSHS with Euler field and dimension

$$
E=2 y_{2} \frac{\partial}{\partial y_{2}}=2 \mathfrak{y}_{2} \frac{\partial}{\partial \mathfrak{y}_{2}}, \quad D=\operatorname{dim} \mathbb{F}_{3}=3 .
$$


Remark 3.1 The superpotentials (42) and (43) have isolated nondegenerate critical points, and so the small quantum cohomology algebra of $\mathbb{F}_{3}$ and the small quantum orbifold cohomology algebra of $\mathbb{P}(1,1,1,3)$ are semisimple.

Remark 3.2 The mirror $D$-module develops a singularity along the lines $\left\{y_{1}=0\right\}$ and $\left\{y_{2}=0\right\}=\left\{\mathfrak{y}_{2}=0\right\}$. These are the solid lines in Figure 3. It is nonsingular along the (dashed) line $\left\{\mathfrak{y}_{1}=0\right\}$.

\subsection{An opposite subspace at each cusp}

We now characterize those opposite subspaces for the B-model VSHS which give rise, via mirror symmetry, to the big quantum cohomology Frobenius manifolds for $\mathbb{F}_{3}$ and for $\mathbb{P}(1,1,1,3)$. As we will see in the next section, these opposite subspaces are not mapped into each other under parallel transport from cusp to cusp.

The $I$-functions (39), (41) define $D$-module homomorphisms

$$
\begin{aligned}
& \mathbb{I}_{\mathbb{F}_{3}}: \mathcal{E} \longrightarrow H^{\bullet}\left(\mathbb{F}_{3}\right) \otimes \mathcal{O}_{\widetilde{\mathcal{M}}_{\mathrm{B}}}\left\{z, z^{-1}\right\}, \\
& \mathbb{I}_{\mathbb{P}(1,1,1,3)}:\left.\mathcal{E}\right|_{\mathcal{M}_{\mathbb{P}(1,1,1,3)} \longrightarrow H_{\mathrm{orb}}(\mathbb{P}(1,1,1,3)) \otimes \mathcal{O}_{\widetilde{\mathcal{M}}_{\mathbb{P}(1,1,1,3)}}\left\{z, z^{-1}\right\}}
\end{aligned}
$$

where we give the right-hand sides the trivial $D$-module structure. These homomorphisms are defined using the isomorphism (36), by sending a representative $P(y, z \partial, z) \in \mathcal{O}_{\mathcal{M}_{\mathrm{B}}}\{z\}\left\langle z \mathcal{T}_{\mathcal{M}_{\mathrm{B}}}\right\rangle$ to $z^{-1} P I_{\mathbb{F}_{3}}$ or to $z^{-1} P I_{\mathbb{P}(1,1,1,3)}$. This does not depend on our choice of representative $P$ since the $I$-functions satisfy the PicardFuchs equations (38), (40). $\mathbb{I}_{\mathbb{F}_{3}}$ is a priori defined only in a small neighbourhood of the cusp for $\mathbb{F}_{3}$, but it can be extended to the whole of $\widetilde{\mathcal{M}}_{\mathrm{B}}$ by analytic continuation (or, which amounts to the same thing, by solving the Picard-Fuchs equations on this larger region). The maps (45) send $\nabla^{z}$-parallel sections of $\mathcal{E}$ to constant sections, and thus identify the space $\mathcal{H}$ of flat sections of $\mathcal{E}$ with Givental's symplectic vector space (with $Q=1$ ):

$$
\begin{aligned}
& \mathbb{I}_{\mathbb{F}_{3}}: \mathcal{H} \cong H^{\bullet}\left(\mathbb{F}_{3}\right) \otimes \mathbb{C}\left\{z, z^{-1}\right\}=\left.\mathcal{H}_{\mathbb{F}_{3}}\right|_{Q_{i}=1} \\
& \mathbb{I}_{\mathbb{P}(1,1,1,3)}: \mathcal{H} \cong H_{\text {orb }}^{\bullet}(\mathbb{P}(1,1,1,3)) \otimes \mathbb{C}\left\{z, z^{-1}\right\}=\left.\mathcal{H}_{\mathbb{P}(1,1,1,3)}\right|_{Q=1}
\end{aligned}
$$

As discussed in Section 2.5.2, the mirror isomorphism (37) sends the generator $\left[\exp \left(W_{y} / z\right) \omega_{y}\right]$ of $\mathcal{E}$ to the family of vectors $y \mapsto I(y, z)=J(q, z)$ lying on Givental's Lagrangian submanifold $\mathcal{L}$. The identifications (46) are exactly those induced by (37). An easy calculation using the explicit forms of the $I$-functions yields:

Proposition 3.3 Under the identifications (46), the B-model grading operator (44) corresponds to the A-model grading operator (22). 
The Hodge structure $\mathbb{E}_{y}$ near cusps behaves as follows. As $\left(y_{1}, y_{2}\right) \rightarrow 0$, we have:

$$
\mathbb{I}_{\mathbb{F}_{3}}\left(\mathbb{E}_{y}\right) \sim e^{\left(p_{1} \log y_{1}+p_{2} \log y_{2}\right) / z}\left(H^{\bullet}\left(\mathbb{F}_{3}\right) \otimes \mathbb{C}\{z\}+O\left(y_{1}, y_{2}\right)\right)
$$

and as $\mathfrak{y}_{2} \rightarrow 0$ with $\mathfrak{y}_{1}=0$, we have:

$$
\mathbb{I}_{\mathbb{P}(1,1,1,3)}\left(\mathbb{E}_{y}\right) \sim e^{3 p \log \mathfrak{y}_{2} / z}\left(H_{\text {orb }}^{\bullet}(\mathbb{P}(1,1,1,3)) \otimes \mathbb{C}\{z\}+O\left(\mathfrak{y}_{2}\right)\right)
$$

These are semi-infinite analogs of Schmid's Nilpotent Orbit Theorem [49]—-the singularity of the Hodge structure near a cusp is asymptotically given by the exponential of nilpotent operators. This corresponds to the fact that $\mathcal{E}$ has quasi-unipotent monodromy at each cusp, and so has a regular singular extension (Deligne's extension) on a finite cover of a neighbourhood of each cusp.

Definition 3.4 The limiting Hodge structure at a cusp $c$ is defined to be a subspace $\mathbb{E}_{c}^{\lim }$ of $\mathcal{H}$ satisfying

$$
\mathbb{E}_{\left(x_{1}, x_{2}\right)} \sim \exp \left(\sum_{i=1}^{2} N_{i} \log x_{i}\right)\left(\mathbb{E}_{c}^{\lim }+O\left(x_{1}, x_{2}\right)\right)
$$

where $N_{i}$ are nilpotent operators and $x_{1}, x_{2}$ are local coordinates centered at $c$ such that $\mathcal{E}$ has a logarithmic singularity along the $x_{i}$-axes. The limiting Hodge structure actually depends on the choice of such coordinates ${ }^{14}$, but in our examples we take $\left(x_{1}, x_{2}\right)=\left(y_{1}, y_{2}\right)$ near the cusp $c_{1}$ for $\mathbb{F}_{3}$ and $\left(x_{1}, x_{2}\right)=\left(\mathfrak{y}_{1}, \mathfrak{y}_{2}\right)$ near the cusp $c_{2}$ of $\mathbb{P}(1,1,1,3)$.

The above calculation shows that:

$$
\begin{aligned}
\mathbb{I}_{\mathbb{F}_{3}}\left(\mathbb{E}_{c_{1}}^{\lim }\right) & =H^{\bullet}\left(\mathbb{F}_{3}\right) \otimes \mathbb{C}\{z\}=\left.\mathcal{H}_{\mathbb{F}_{3}}^{+}\right|_{Q_{i}=1} \\
\mathbb{I}_{\mathbb{P}(1,1,1,3)}\left(\mathbb{E}_{c_{2}}^{\lim }\right) & =H_{\text {orb }}^{\bullet}(\mathbb{P}(1,1,1,3)) \otimes \mathbb{C}\{z\}=\mathcal{H}_{\mathbb{P}(1,1,1,3)}^{+} \mid Q=1
\end{aligned}
$$

We now construct an opposite subspace $\mathcal{H}_{-}$for each cusp $c$. We postulate that $\mathcal{H}_{-}$ should satisfy:

(a) $\mathcal{H}_{-}$is opposite to the limiting Hodge structure. $\mathbb{E}_{c}^{\text {lim }}$

(b) $\mathcal{H}_{-}$is preserved by the grading operator $\mathrm{Gr}$.

(c) $\mathcal{H}_{-}$is invariant under local monodromy, and moreover the monodromy action $M$ satisfies $M^{N}=\mathrm{id}$ on $z \mathcal{H}_{-} / \mathcal{H}_{-}$where $N$ is the order of the local isotropy group at the cusp $c$.

${ }^{14}$ A coordinate change of the form $\log x_{i}^{\prime}=\log x_{i}+f_{i}\left(x_{1}, x_{2}\right)$ with $f_{i}(0,0)=0$ does not change the limiting Hodge structure, so $\mathbb{E}_{c}^{\lim }$ depends only on the choice of "origin" of $\log x_{i}$. 
For $\mathbb{F}_{3}$, the local monodromy means the monodromy around the axes $y_{1}=0$ and $y_{2}=0$. The corresponding monodromy actions on $\mathcal{H}_{\mathbb{F}_{3}}$ are given by

$$
M_{1}=\exp \left(2 \pi \mathrm{i} p_{1} / z\right), \quad M_{2}=\exp \left(2 \pi \mathrm{i} p_{2} / z\right) .
$$

For $\mathbb{P}(1,1,1,3)$, the local monodromy means the monodromy coming from an orbifold loop $[0,1] \ni t \mapsto\left(\mathfrak{y}_{1}, \mathfrak{y}_{2}\right)=\left(0, e^{2 \pi \mathrm{i} t / 3}\right)$. The corresponding action on $\mathcal{H}_{\mathbb{P}(1,1,1,3)}$ is given by

$$
M=M_{0} \exp (2 \pi i p / z)
$$

where $\left.\quad M_{0}\right|_{H \bullet(\mathbb{P}(1,1,1,3))}=\mathrm{id}$

$M_{0}\left(\mathbf{1}_{1 / 3}\right)=\alpha \mathbf{1}_{1 / 3}$,

$M_{0}\left(\mathbf{1}_{2 / 3}\right)=\alpha^{2} \mathbf{1}_{2 / 3}$

and $\alpha=\exp (2 \pi i / 3)$. We have $N=1$ for $\mathbb{F}_{3}$ and $N=3$ for $\mathbb{P}(1,1,1,3)$. Condition (c) above implies that the connection 1-form $A$ in Proposition 2.11 is well-defined on an $N$-fold cover (uniformizing system) of a neighbourhood of each cusp.

Theorem 3.5 Let $c_{1}$ and $c_{2}$ be the cusps of $\mathcal{M}_{\mathrm{B}}$ corresponding to $\mathbb{F}_{3}$ and $\mathbb{P}(1,1,1,3)$ respectively. For each $c_{i}$ there exists a unique opposite subspace $\mathcal{H}_{c_{i}}^{-}$satisfying conditions (a)-(c) above. Moreover, $\mathcal{H}_{c_{i}}^{-}$corresponds under (46) to the standard A-model opposite subspace:

$$
\begin{aligned}
\mathbb{I}_{\mathbb{F}_{3}}\left(\mathcal{H}_{c_{1}}^{-}\right) & =z^{-1} H^{\bullet}\left(\mathbb{F}_{3}\right) \otimes \mathcal{O}\left(\mathbb{P}^{1} \backslash\{0\}\right)=\left.\mathcal{H}_{\mathbb{F}_{3}}^{-}\right|_{Q_{i}=1} \\
\mathbb{I}_{\mathbb{P}(1,1,1,3)}\left(\mathcal{H}_{c_{2}}^{-}\right) & =z^{-1} H_{\text {orb }}^{\bullet}(\mathbb{P}(1,1,1,3)) \otimes \mathcal{O}\left(\mathbb{P}^{1} \backslash\{0\}\right)=\left.\mathcal{H}_{\mathbb{P}(1,1,1,3)}^{-}\right|_{Q=1}
\end{aligned}
$$

Proof We give a proof only for $\mathbb{P}(1,1,1,3)$. The $\mathbb{F}_{3}$ case is similar and easier. Throughout the proof we identify $\mathcal{H}$ with $\left.\mathcal{H}_{\mathbb{P}(1,1,1,3)}\right|_{Q=1}$ via the map $\mathbb{I}_{\mathbb{P}(1,1,1,3)}$ and write $\mathcal{H}_{-}$for $\mathbb{I}_{\mathbb{P}(1,1,1,3)} \mathcal{H}_{c_{2}}^{-}$.

Proposition 3.3 implies that the A-model grading operator (22) preserves $\mathcal{H}_{-}$:

$$
\mathrm{Gr}=2 z \partial_{z}+\mathrm{Gr}_{0}-2 c_{1}(\mathbb{P}(1,1,1,3)) / z, \quad c_{1}(\mathbb{P}(1,1,1,3))=6 p .
$$

On the other hand, the logarithm $6 \pi i p / z=\log \left(M^{3}\right)$ of the cube of the monodromy preserves $\mathcal{H}_{-}$, and so the "usual" grading operator $2 z \partial_{z}+\mathrm{Gr}_{0}$ also preserves $\mathcal{H}_{-}$. This means that $\mathcal{H}_{-}$is a homogeneous subspace of $\mathcal{H}$. Because $\mathcal{H}_{-}$is opposite to $\mathbb{E}_{c_{2}}^{\lim }$, there is a unique $\mathbb{C}$-basis $\left\{\psi_{0}, \ldots, \psi_{3}, \phi_{1}, \phi_{2}\right\}$ of $z \mathcal{H}_{-} \cap \mathbb{E}_{c_{2}}^{\lim }$ such that:

$$
\psi_{i}=p^{i}+O(z), \quad \phi_{1}=\mathbf{1}_{1 / 3}+O(z), \quad \phi_{2}=\mathbf{1}_{2 / 3}+O(z)
$$

These elements must be homogeneous. Since both $M$ and $\exp (2 \pi i p / z)$ preserve $\mathcal{H}_{-}, M_{0}$ must also preserve $\mathcal{H}_{-}$. It is clear that $M_{0}$ preserves $\mathbb{E}_{c_{2}}^{\lim }$, so it acts on $z \mathcal{H}_{-} \cap \mathbb{E}_{c_{2}}^{\lim }$. Thus $z \mathcal{H}_{-} \cap \mathbb{E}_{c_{2}}^{\lim }$ decomposes into eigenspaces for $M_{0}$; it follows that 
$\psi_{i} \in H^{\bullet}(\mathbb{P}(1,1,1,3))\{z\}$ and $\phi_{i} \in H^{\bullet}\left(\mathbb{P}\left(V^{i / 3}\right)\right)\{z\}$. Homogeneity now implies that $\phi_{i}=\mathbf{1}_{i / 3}$.

Since $M^{3}$ acts trivially on $z \mathcal{H}_{-} / \mathcal{H}_{-}$it follows that $\frac{1}{3} \log M^{3}$ sends $z \mathcal{H}_{-}$to $\mathcal{H}_{-}$. Thus $\frac{1}{3} z \log M^{3}=2 \pi i p$ preserves $\mathbb{E}_{c_{2}}^{\lim }$ and $z \mathcal{H}_{-}$simultaneously, and therefore acts on $z \mathcal{H}_{-} \cap \mathbb{E}_{c_{2}}^{\lim }$. By homogeneity again, we can write

$$
\psi_{i}=p^{i}+\sum_{j=0}^{i-1} c_{i j} z^{i-j} p^{j}
$$

for some $c_{i j} \in \mathbb{C}$. As $p^{4-i} \psi_{i} \in z \mathcal{H}_{-} \cap \mathbb{E}_{c_{2}}^{\lim }$ and $p^{4-i} \psi_{i}$ is divisible by $z$, we know that $p^{4-i} \psi_{i}$ should be zero. This shows that $c_{i j}=0$ and that $\psi_{i}=p^{i}$. Since $z \mathcal{H}_{-}$is spanned over $\mathcal{O}\left(\mathbb{P}^{1} \backslash\{0\}\right)$ by $\psi_{0}, \ldots, \psi_{3}, \phi_{1}, \phi_{2}$, it follows that $\mathcal{H}_{-}=$ $\mathcal{H}_{\mathbb{P}(1,1,1,3)}^{-} \mid Q=1$.

Monodromy properties also force, as we now show, the A-model and B-model pairings to coincide up to an overall scalar factor. In the next section we will see that the composition $\mathbb{I}_{\mathbb{F}_{3}} \circ \mathbb{I}_{\mathbb{P}(1,1,1,3)}^{-1}$ exactly preserves Givental's symplectic form.

Proposition 3.6 Under the identifications (46), the B-model symplectic form corresponds to a scalar multiple of Givental's symplectic form. In particular, $\mathcal{H}_{c_{i}}^{-}$is isotropic with respect to the $B$-model symplectic form.

Proof We give a proof only for $\mathbb{P}(1,1,1,3)$; the $\mathbb{F}_{3}$ case is similar. Let $(\cdot, \cdot)$ be the $\mathbb{C}\left\{z, z^{-1}\right\}$-valued pairing on $\left.\mathcal{H}_{\mathbb{P}(1,1,1,3)}\right|_{Q=1}$ induced by the B-model pairing. The definition of the $\mathrm{B}$-model pairing shows that this is monodromy-invariant:

$$
(M \alpha, M \beta)_{\mathrm{B}}=(\alpha, \beta)_{\mathrm{B}}
$$

Using $6 \pi$ i $p / z=\log M^{3}$, we have

$$
(p \alpha, \beta)_{\mathrm{B}}=(\alpha, p \beta)_{\mathrm{B}}
$$

and so $M_{0}=M \exp (-2 \pi \mathrm{i} p / z)$ also preserves the pairing $(\cdot, \cdot)$. This implies that

$$
\left(H^{\bullet}(\mathbb{P}(1,1,1,3)), \mathbf{1}_{i / 3}\right)_{\mathrm{B}}=0 \quad \text { and } \quad\left(\mathbf{1}_{i / 3}, \mathbf{1}_{i / 3}\right)_{\mathrm{B}}=0
$$

for $i=1,2$.

From the asymptotics (48) we know that for each $\left.\alpha \in \mathcal{H}_{\mathbb{P}(1,1,1,3)}^{+}\right|_{Q=1}$, there exists a family of elements $\left\{\alpha_{\mathfrak{y}_{2}}\right\}$ in $\left.\mathcal{H}_{\mathbb{P}(1,1,1,3)}\right|_{Q=1}$ such that

$$
\alpha_{\mathfrak{y}_{2}}=\exp \left(3 p \log \mathfrak{y}_{2} / z\right)\left(\alpha+O\left(\mathfrak{y}_{2}\right)\right) \in \mathbb{E}_{\mathfrak{y}_{1}=0, \mathfrak{y}_{2}} .
$$


For any $\alpha, \beta \in \mathcal{H}_{\mathbb{P}(1,1,1,3)}^{+}$, the B-model pairing of $\alpha_{\mathfrak{y}_{2}}$ and $\beta_{\mathfrak{y}_{2}}$ takes values in $\mathbb{C}\{z\}$ :

$$
\left(\alpha_{\mathfrak{y}_{2}}, \beta_{\mathfrak{y}_{2}}\right)_{\mathrm{B}}=\left(\alpha+O\left(\mathfrak{y}_{2}\right), \beta+O\left(\mathfrak{y}_{2}\right)\right)_{\mathrm{B}} \in \mathbb{C}\{z\} .
$$

Taking the limit $\mathfrak{y}_{2} \rightarrow 0$, we see that $(\alpha, \beta)_{\mathrm{B}}$ is in $\mathbb{C}\{z\}$.

The compatibility of grading and the pairing gives that for homogeneous elements $\alpha, \beta \in H_{\text {orb }}^{\bullet}(\mathbb{P}(1,1,1,3))$ we have:

$2 z \partial_{z}(\alpha, \beta)_{\mathrm{B}}=(\operatorname{deg} \alpha+\operatorname{deg} \beta-6)(\alpha, \beta)_{\mathrm{B}}$

$$
-\left(\left(c_{1}(\mathcal{X}) / z\right) \cup \alpha, \beta\right)_{\mathrm{B}}-\left(\alpha,\left(c_{1}(\mathcal{X}) / z\right) \cup \beta\right)_{\mathrm{B}}
$$

The second line vanishes by (49). This means that $(\alpha, \beta)_{\mathrm{B}}$ is homogeneous of degree $\operatorname{deg} \alpha+\operatorname{deg} \beta-6$. From this homogeneity, Equation (49), the orthogonality (50), and the fact that $(\alpha, \beta)_{\mathrm{B}} \in \mathbb{C}\{z\}$, it follows that the only nonvanishing pairings among basis elements are

$$
\left(p^{i}, p^{j}\right)_{\mathrm{B}}=\left(\mathbf{1}, p^{i+j}\right)_{\mathrm{B}} \in \mathbb{C}, \quad\left(\mathbf{1}_{1 / 3}, \mathbf{1}_{2 / 3}\right)_{\mathrm{B}}=\left(\mathbf{1}_{2 / 3}, \mathbf{1}_{1 / 3}\right)_{\mathrm{B}} \in \mathbb{C}
$$

with $i+j=3$. This shows directly that $\mathcal{H}_{\mathbb{P}(1,1,1,3)}^{-}$is isotropic with respect to the $\mathrm{B}$-model symplectic form. But the general theory of VSHS in Section 2.2 implies that the B-model pairing also satisfies (see Equation (11))

$$
\left(\alpha \circ_{q} \beta, \gamma\right)_{\mathrm{B}}=\left(\alpha, \beta \circ_{q} \gamma\right)_{\mathrm{B}} \quad \text { for all } \alpha, \beta, \gamma \in H_{\mathrm{orb}}^{\bullet}(\mathbb{P}(1,1,1,3))
$$

because the small quantum orbifold cohomology algebra is generated by $p$. Thus $(\cdot, \cdot)$ is completely determined by the value $\left(1, p^{3}\right)_{\mathrm{B}}$ and is proportional to the orbifold Poincaré pairing.

Remark 3.7 In proving the uniqueness in general of opposite subspaces and pairings which behave well under monodromy, the hard Lefschetz property of the usual cohomology of a projective orbifold will play an important role. This will be explained in Iritani [38]. See also Iritani [37, Theorem 3.13] for the uniqueness of opposite subspaces in the A-model and Iritani [36, Appendix] for the matching of the A-model and $\mathrm{B}-$ model pairings for a general compact weak Fano toric orbifold. In the proofs above, we implicitly used the hard Lefschetz property of $H^{\bullet}(\mathbb{P}(1,1,1,3))$. A hard Lefschetz property for orbifold cohomology is discussed in Theorem 5.10 below.

Definition 3.8 A polarization of $\mathcal{H}$ at a cusp $c$ is a decomposition

$$
\mathcal{H}=\mathbb{E}_{c}^{\lim } \oplus \mathcal{H}_{c}^{-}
$$

where $\mathbb{E}_{c}^{\lim }$ is the limiting Hodge structure and $\mathcal{H}_{c}^{-}$is an opposite subspace. 
The polarization at a cusp will be mapped by $\mathbb{I}_{\mathbb{F}_{3}}$ or $\mathbb{I}_{\mathbb{P}(1,1,1,3)}$ to the standard polarization:

$$
\left.\mathcal{H}_{\mathbb{F}_{3}}^{+} \oplus \mathcal{H}_{\mathbb{F}_{3}}^{-}\right|_{Q_{i}=1} \quad \text { or }\left.\quad \mathcal{H}_{\mathbb{P}(1,1,1,3)}^{+} \oplus \mathcal{H}_{\mathbb{P}(1,1,1,3)}^{-}\right|_{Q=1}
$$

\subsection{The polarizations are different}

We now compare the polarizations at the cusps of $\mathcal{M}_{\mathrm{B}}$ corresponding to $\mathbb{F}_{3}$ and to $\mathbb{P}(1,1,1,3)$. Let $\overline{\mathbb{U}}:\left.\left.\mathcal{H}_{\mathbb{P}(1,1,1,3)}\right|_{Q=1} \rightarrow \mathcal{H}_{\mathbb{F}_{3}}\right|_{Q_{i}=1}$ be the linear transformation defined by the composition

$$
\left.\left.\mathcal{H}_{\mathbb{P}(1,1,1,3)}\right|_{Q=1} \stackrel{\left(\mathbb{I}_{\mathbb{P}(1,1,1,3)}\right)^{-1}}{\longrightarrow} \mathcal{H} \stackrel{\mathbb{I}_{\mathbb{F}_{3}}}{\longrightarrow} \mathcal{H}_{\mathbb{F}_{3}}\right|_{Q_{i}=1}
$$

and let $\mathbb{U}:\left.\left.\mathcal{H}_{\mathbb{P}(1,1,1,3)}\right|_{Q=1} \rightarrow \mathcal{H}_{\mathbb{F}_{3}}\right|_{Q_{i}=1}$ be $\overline{\mathbb{U}}$ followed by changing the sign of $z$. The transformation $\mathbb{U}$ (or equivalently $\mathbb{U}$ ) measures the difference between the polarizations at the two cusps. As we will see, the sign flip in the definition of $\mathbb{U}$ comes from the sign flip which relates the A-model VSHS to the tangent spaces to Givental's Lagrangian submanifold (see Section 2.3.3).

Proposition 3.9 The matrix of $\mathbb{U}$ with respect to the bases for $H_{\mathrm{orb}}^{\bullet}(\mathbb{P}(1,1,1,3))$ and $H^{\bullet}\left(\mathbb{F}_{3}\right)$ defined in Section 2.1.1 is:

$$
\left(\begin{array}{cccccc}
1 & 0 & 0 & 0 & 0 & 0 \\
0 & 1 & 0 & 0 & 0 & 0 \\
0 & 0 & 1 & 0 & 0 & 0 \\
0 & 0 & 0 & 0 & \frac{2 \sqrt{3} \pi}{3 \Gamma(1 / 3)^{3}} z & \frac{2 \sqrt{3} \pi}{3 \Gamma(2 / 3)^{3}} \\
-\frac{\pi^{2}}{3 z^{2}} & 0 & 0 & 0 & \frac{2 \pi^{2}}{3 \Gamma(1 / 3)^{3}} & -\frac{2 \pi^{2}}{3 \Gamma(2 / 3)^{3} z} \\
\frac{8 \zeta(3)}{z^{3}} & 0 & 0 & 1 & \frac{2 \sqrt{3} \pi^{3}}{9 \Gamma(1 / 3)^{3} z} & \frac{2 \sqrt{3} \pi^{3}}{9 \Gamma(2 / 3)^{3} z^{2}}
\end{array}\right)
$$

Here $\zeta$ is the Riemann zeta function. The linear transformation $\mathbb{U}$ preserves the grading and the symplectic forms but does not preserve the standard opposite subspaces.

Proof The $I$-functions $I_{\mathbb{F}_{3}}$ and $I_{\mathbb{P}(1,1,1,3)}$ are the images under the maps $\mathbb{I}_{\mathbb{F}_{3}}$ and $\mathbb{I}_{\mathbb{P}(1,1,1,3)}$ of the generator $\left[\exp \left(W_{y} / z\right) \omega_{y}\right] \in \mathcal{E}$. It follows that

$$
\overline{\mathbb{U}}\left(I_{\mathbb{P}(1,1,1,3)}\right)=\left.I_{\mathbb{F}_{3}}\right|_{\mathfrak{y}_{1}=0}
$$

where we regard $I_{\mathbb{P}(1,1,1,3)}$ as a function of $\mathfrak{y}_{2}$ via the map $y=\mathfrak{y}_{2}^{3}$ discussed above Figure 3 . We calculate $\overline{\mathbb{U}}$ (and hence $\mathbb{U}$ ) by analytically continuing $I_{\mathbb{F}_{3}}$ to a neighbourhood of the large radius limit point for $\mathbb{P}(1,1,1,3)$ and then comparing it with 
$I_{\mathbb{P}(1,1,1,3)}$. Using the Barnes method (see the Appendix), one finds:

$$
\begin{aligned}
& I_{\mathbb{F}_{3}}\left(\mathfrak{y}_{1}, \mathfrak{y}_{2}, z\right)=z \Gamma\left(1+\frac{p_{1}}{z}\right)^{3} \Gamma\left(1+\frac{p_{2}}{z}\right) \Gamma\left(1+\frac{p_{2}-3 p_{1}}{z}\right) \times \\
& \sum_{k, l \geq 0} \frac{(-1)^{k+l} \sin \left(\frac{p_{2}-3 p_{1}}{z} \pi\right)}{3 \sin \left(\frac{p_{2}-3 p_{1}}{3 z} \pi+\frac{l-k}{3} \pi\right)} \frac{\mathfrak{y}_{1}^{k} \mathfrak{y}_{2}^{l+p_{2} / z}}{k ! z^{2 l} \Gamma\left(1+\frac{p_{2}}{3 z}+\frac{l-k}{3}\right)^{3} \Gamma\left(1+\frac{p_{2}}{z}+l\right)}
\end{aligned}
$$

We compare this with:

$$
I_{\mathbb{P}(1,1,1,3)}\left(\mathfrak{y}_{2}, z\right)=z \sum_{m \geq 0} \frac{\Gamma\left(1-\left\langle-\frac{m}{3}\right\rangle+\frac{p}{z}\right)^{3} \Gamma\left(1+\frac{3 p}{z}\right)}{\Gamma\left(1+\frac{p}{z}+\frac{m}{3}\right)^{3} \Gamma\left(1+\frac{3 p}{z}+m\right)} \frac{\mathfrak{y}_{2}^{m+3 p / z}}{z^{2 m}} \frac{\mathbf{1}_{\langle m / 3\rangle}}{z^{3\langle-m / 3\rangle}} .
$$

Since $\overline{\mathbb{U}}$ is equivariant with respect to the monodromy action around the axis $\left\{y_{2}=0\right\}=$ $\left\{\mathfrak{y}_{2}=0\right\}$, we have $\overline{\mathbb{U}} e^{2 \pi i 3 p / z}=e^{2 \pi i p_{2} / z} \overline{\mathbb{U}}$ and so $\overline{\mathbb{U}} 3 p=p_{2} \overline{\mathbb{U}}$. Thus:

$$
\begin{aligned}
\overline{\mathbb{U}}\left(\mathbf{1}_{0}\right) & =\frac{\Gamma\left(1+\frac{p_{1}}{z}\right)^{3} \Gamma\left(1+\frac{\mathfrak{p}_{1}}{z}\right)}{\Gamma\left(1+\frac{p_{2}}{3 z}\right)^{3}} \frac{\sin \left(\frac{\mathfrak{p}_{1}}{z} \pi\right)}{3 \sin \left(\frac{\mathfrak{p}_{1}}{3 z} \pi\right)} \\
z^{-1} \overline{\mathbb{U}}\left(\mathbf{1}_{2 / 3}\right) & =\frac{\Gamma\left(1+\frac{p_{1}}{z}\right)^{3} \Gamma\left(1+\frac{\mathfrak{p}_{1}}{z}\right)}{\Gamma\left(\frac{2}{3}+\frac{p_{2}}{3 z}\right)^{3}} \frac{\sin \left(\frac{\mathfrak{p}_{1}}{z} \pi\right)}{3 \sin \left(\frac{\mathfrak{p}_{1}}{3 z} \pi+\frac{2}{3} \pi\right)} \\
z^{-2} \overline{\mathbb{U}}\left(\mathbf{1}_{1 / 3}\right) & =-\frac{\Gamma\left(1+\frac{p_{1}}{z}\right)^{3} \Gamma\left(1+\frac{\mathfrak{p}_{1}}{z}\right)}{\Gamma\left(\frac{1}{3}+\frac{p_{2}}{3 z}\right)^{3}} \frac{\sin \left(\frac{\mathfrak{p}_{1}}{z} \pi\right)}{3 \sin \left(\frac{\mathfrak{p}_{1}}{3 z} \pi+\frac{1}{3} \pi\right)}
\end{aligned}
$$

where $\mathfrak{p}_{1}=p_{2}-3 p_{1}$, and the conclusion follows. The value $\zeta(3)$ in the matrix for $\mathbb{U}$ comes from the expansion of the $\Gamma$-function:

$$
\Gamma(1+x)=\exp \left(-\gamma x+\frac{\pi^{2}}{12} x^{2}-\frac{\zeta(3)}{3} x^{3}+O\left(x^{4}\right)\right)
$$

where $\gamma$ is Euler's constant. The transformation $\mathbb{U}$ does not map $\mathcal{H}_{\mathbb{P}(1,1,1,3)}^{-}$to $\mathcal{H}_{\mathbb{F}_{3}}^{-}$ because the matrix (51) contains strictly positive powers of $z$.

Remark 3.10 The symplectic transformation $\mathbb{U}$ always has an ambiguity due to the monodromy action on $\mathcal{H}$. This corresponds to the choice of branch cuts in the process of analytic continuation.

Remark 3.11 A closely-related symplectic transformation (with $z=1$ ) occurs in work of Aganagic-Bouchard-Klemm [3, Equation 6.21]. They studied a phase-transition from local $\mathbb{P}^{2}$ (the total space of the canonical bundle $K_{\mathbb{P}^{2}}$ ) to $\mathbb{C}^{3} / \mathbb{Z}_{3}$. Our example here is a global version of this but is not Calabi-Yau. 


\subsection{The proof of Conjecture 1.3}

In Theorem 3.12 below, we prove Conjecture 5.1 when $\mathcal{X}=\mathbb{P}(1,1,1,3)$ and $Y=\mathbb{F}_{3}$. Conjecture 1.3 in the Introduction follows from this and the definition (26) of the analytic Givental's cone.

Theorem 3.12 Let $\left.\mathbb{E}_{\tau}^{\mathbb{F}_{3}} \subset \mathcal{H}_{\mathbb{F}_{3}}\right|_{Q_{i}=1}$ and $\left.\mathbb{E}_{\tau}^{\mathbb{P}(1,1,1,3)} \subset \mathcal{H}_{\mathbb{P}(1,1,1,3)}\right|_{Q=1}$ be the moving subspace realizations (25) of the analytic big A-model VSHSs of $\mathbb{F}_{3}$ and $\mathbb{P}(1,1,1,3)$. Then there exists a map $\Upsilon$ from an open subset of $H_{\text {orb }}^{\bullet}(\mathbb{P}(1,1,1,3))$ to an open subset of $H^{\bullet}\left(\mathbb{F}_{3}\right)$ such that, after analytic continuation if necessary,

$$
\overline{\mathbb{U}}\left(\mathbb{E}_{\tau}^{\mathbb{P}(1,1,1,3)}\right)=\mathbb{E}_{\Upsilon(\tau)}^{\mathbb{F}_{3}}
$$

where $\mathbb{U}$ is the symplectic transformation from Proposition 3.9.

Proof We use Dubrovin's Reconstruction Theorem [21]. This implies that the Frobenius manifold given by big quantum (orbifold) cohomology can be uniquely reconstructed from one semisimple fiber as an isomonodromic deformation of the differential equation

$$
\left[\frac{1}{2} \mathrm{Gr}-\frac{1}{z} \nabla_{E}^{z}\right] \psi=\left[z \frac{\partial}{\partial z}-\frac{1}{z} E \bullet_{\tau}+\frac{1}{2} \mathrm{Gr}_{0}\right] \psi=0
$$

where $\mathrm{Gr}, \mathrm{Gr}_{0}$ are grading operators. We know from the mirror analysis that the small quantum cohomology algebras of $\mathbb{F}_{3}$ and $\mathbb{P}(1,1,1,3)$ are semisimple. The big quantum cohomologies of $\mathbb{F}_{3}$ and $\mathbb{P}(1,1,1,3)$ are therefore determined as analytic Frobenius manifolds by the small quantum cohomologies.

Write $\mathcal{X}=\mathbb{P}(1,1,1,3)$ and $Y=\mathbb{F}_{3}$. Let $\operatorname{mir}_{Y}: \widetilde{\mathcal{M}}_{\mathrm{B}} \rightarrow H^{2}(Y)$ and $\operatorname{mir}_{\mathcal{X}}: \widetilde{\mathcal{M}}_{\mathrm{B}} \supset$ $\widetilde{\mathcal{M}}_{\mathcal{X}} \rightarrow H^{2}(\mathcal{X})$ be (analytic continuations of) the mirror maps. They are given by the coefficients of $z^{0}$ of the $I$-functions $I_{\mathbb{F}_{3}}, I_{\mathbb{P}(1,1,1,3)}$ in equations (39), (41). The mirror theorems discussed in Sections 3.2, 3.4 imply that

$$
\mathbb{I}_{Y}\left(\mathcal{E}_{y}\right)=\mathbb{E}_{\operatorname{mir}_{Y}(y)}^{Y} \quad \text { and } \quad \mathbb{I}_{\mathcal{X}}\left(\mathcal{E}_{y}\right)=\mathbb{E}_{\operatorname{mir}_{\mathcal{X}}(y)}^{\mathcal{X}}
$$

for $y \in \widetilde{\mathcal{M}}_{\mathrm{B}}$ and $y \in \widetilde{\mathcal{M}}_{\mathcal{X}}$ respectively. These equations hold a priori in neighbourhoods of the cusps, but hold everywhere by analytic continuation. By the definition of $\overline{\mathbb{U}}$, we have

$$
\overline{\mathbb{U}}\left(\mathbb{E}_{\operatorname{mir}_{\mathcal{X}}(y)}^{\mathcal{X}}\right)=\mathbb{E}_{\operatorname{mir}_{Y}(y)}^{Y}
$$

for $y \in \widetilde{\mathcal{M}}_{\mathcal{X}}$. Take a semisimple point $y_{0} \in \widetilde{\mathcal{M}}_{\mathcal{X}}$ (in fact every point on $\widetilde{\mathcal{M}}_{\mathcal{X}}$ is semisimple) and a small open neighbourhood $U_{0}$ of $y_{0}$ in $\widetilde{\mathcal{M}}_{\mathcal{X}}$. Since the B-model 
and A-model grading operators match (Proposition 3.3) and the Euler vector field is tangent to $\widetilde{\mathcal{M}}_{\mathcal{X}}, \overline{\mathbb{U}}$ induces an isomorphism of graded VSHSs:

$$
\left.\left.\mathcal{E}_{\mathrm{A}}^{\mathcal{X}, \text { big }}\right|_{\operatorname{mir}_{\mathcal{X}}\left(U_{0}\right)} \cong \mathcal{E}_{\mathrm{A}}^{Y \text {,big }}\right|_{\operatorname{mir}_{Y}\left(U_{0}\right)}
$$

Take an isotropic, $\mathrm{Gr}$-invariant opposite subspace $\mathcal{H}^{-}$of $\mathcal{E}$ at $y_{0}$. This gives rise to the opposite subspaces $\mathbb{I}_{\mathcal{X}}\left(\mathcal{H}^{-}\right)$and $\mathbb{I}_{Y}\left(\mathcal{H}^{-}\right)$of $\mathcal{E}_{\mathrm{A}}^{\mathcal{X} \text {, big }}$ and $\mathcal{E}_{\mathrm{A}}^{Y \text {,big }}$-these subspaces are opposite in neighbourhoods of $\sigma_{0}:=\operatorname{mir}_{\mathcal{X}}\left(y_{0}\right)$ and $\tau_{0}:=\operatorname{mir}_{Y}\left(y_{0}\right)$ respectively-and produces Frobenius manifold structures ${ }^{15}$ on the analytic germs $\left(H_{\text {orb }}^{\bullet}(\mathcal{X} ; \mathbb{C}), \tau_{0}\right)$ and $\left(H^{\bullet}(Y ; \mathbb{C}), \tau_{0}\right)$. Since these two Frobenius manifolds are the unfolding of the same differential equation (53) at $y_{0}$, by Dubrovin's Reconstruction Theorem we have a natural isomorphism of Frobenius manifolds $\Upsilon:\left(H_{\text {orb }}^{\bullet}(\mathcal{X} ; \mathbb{C}), \sigma_{0}\right) \cong\left(H^{\bullet}(Y ; \mathbb{C}), \tau_{0}\right)$. Forgetting the opposite subspace, we conclude that there is an isomorphism of the underlying VSHSs, ie that the isomorphism (55) extends to open neighbourhoods of $\sigma_{0} \in H_{\text {orb }}^{\bullet}(\mathcal{X} ; \mathbb{C})$ and $\tau_{0} \in H^{\bullet}(Y ; \mathbb{C})$. The moving subspace realizations of the two big A-model VSHSs are therefore related by a constant $\mathbb{C}\left\{z, z^{-1}\right\}$-linear transformation. Equation (54) shows that this transformation is $\overline{\mathbb{U}}$.

Remark 3.13 When reconstructing big quantum cohomology from small quantum cohomology, we could use quantum $H^{2}$-generation in place of Dubrovin's Reconstruction Theorem. In fact, Dubrovin Reconstruction is a special case of quantum $\mathrm{H}^{2}$-generation where the product $E \bullet_{\tau}$ of the Euler vector field generates the total cohomology. In our case, orbifold cohomology $H_{\text {orb }}^{\bullet}(\mathbb{P}(1,1,1,3))$ is not generated by $H^{2}(\mathbb{P}(1,1,1,3))$, but quantum orbifold cohomology is generated by $H^{2}(\mathbb{P}(1,1,1,3))$. Reconstruction theorems of Hertling-Manin [32], Iritani [39, Remark 4.10] and Rose [45] are also applicable here. These are generalizations of the First Reconstruction Theorem of Kontsevich-Manin [40], where classical $\mathrm{H}^{2}$-generation is assumed.

\subsection{The flat coordinates are different}

We can see the difference between the big quantum cohomology Frobenius manifolds for $\mathbb{F}_{3}$ and $\mathbb{P}(1,1,1,3)$ more explicitly as follows. The vectors $I_{\mathbb{F}_{3}}(y,-z)$ and $\mathbb{U}^{-1} I_{\mathbb{F}_{3}}(y,-z)$ are on the cones $\mathcal{L}_{\mathbb{F}_{3}}$ and $\mathcal{L}_{\mathbb{P}(1,1,1,3)}$ respectively. They expand as:

$$
I_{\mathbb{F}_{3}}(y,-z)=-z+\tau_{1} p_{1}+\tau_{2} p_{2}-\left(-\frac{1}{3} \frac{\partial F_{0}}{\partial \tau_{1}} \mathfrak{p}_{1}^{2}+\frac{\partial F_{0}}{\partial \tau_{2}} p_{1}^{2}\right) \frac{1}{z}+O\left(z^{-2}\right)
$$

$$
\mathbb{U}^{-1}\left(I_{\mathbb{F}_{3}}(y,-z)\right)=-z+\mathfrak{t}_{1} \mathbf{1}_{2 / 3}+\mathfrak{t}_{2}(3 p)-\left(3 \frac{\partial F_{0}^{\text {orb }}}{\partial \mathfrak{t}_{1}} \mathbf{1}_{1 / 3}+\frac{\partial F_{0}^{\text {orb }}}{\partial \mathfrak{t}_{2}} p^{2}\right) \frac{1}{z}+O\left(z^{-2}\right)
$$

${ }^{15}$ These Frobenius manifold structures are not in general the quantum cohomology Frobenius manifold structures, because in general $\mathbb{I}_{\mathcal{X}}\left(\mathcal{H}^{-}\right) \neq \mathcal{H}_{\mathcal{X}}^{-}$and $\mathbb{I}_{Y}\left(\mathcal{H}^{-}\right) \neq \mathcal{H}_{Y}^{-}$. 
where $\left(\tau_{1}, \tau_{2}\right)$ and $\left(\mathfrak{t}_{1}, \mathfrak{t}_{2}\right)$ are flat coordinates on $\widetilde{\mathcal{M}}_{\mathrm{B}}$ associated with the quantum cohomologies and $F_{0}, F_{0}^{\text {orb }}$ are the genus-zero Gromov-Witten potentials for $\mathbb{F}_{3}$ and $\mathbb{P}(1,1,1,3)$. Combining (51) and (56), we see that

$$
\tau_{1}=-\frac{2 \sqrt{3} \pi}{3 \Gamma\left(\frac{2}{3}\right)^{3}} \mathfrak{t}_{1}+\frac{2 \sqrt{3} \pi}{\Gamma\left(\frac{1}{3}\right)^{3}} \frac{\partial F_{0}^{\text {orb }}}{\partial \mathfrak{t}_{1}}, \quad \tau_{2}+\frac{1}{3} \tau_{1}=\mathfrak{t}_{2} .
$$

From (52) we find

$$
\mathfrak{t}_{1}=\sum_{n=0}^{\infty}(-1)^{n} \frac{\prod_{k=0}^{n-1}\left(k+\frac{1}{3}\right)^{3}}{(3 n+1) !} \mathfrak{y}_{1}^{3 n+1}, \quad 3 \frac{\partial F_{0}^{\text {orb }}}{\partial \mathfrak{t}_{1}}=\sum_{n=0}^{\infty}(-1)^{n} \frac{\prod_{k=0}^{n-1}\left(k+\frac{2}{3}\right)^{3}}{(3 n+2) !} \mathfrak{y}_{1}^{3 n+2}
$$

and thus:

$$
3 \frac{\partial F_{0}^{\text {orb }}}{\partial \mathfrak{t}_{1}}=\frac{1}{2} \mathfrak{t}_{1}^{2}-\frac{1}{3^{2} \cdot 5 !} \mathfrak{t}_{1}^{5}+\frac{1}{3 \cdot 8 !} \mathfrak{t}_{1}^{8}-\frac{1093}{3^{5} \cdot 11 !} \mathfrak{t}_{1}^{11}+\cdots
$$

Combining (57) and (58) shows that the flat coordinate systems $\left(\tau_{1}, \tau_{2}\right)$ and $\left(\mathfrak{t}_{1}, \mathfrak{t}_{2}\right)$ on $\mathcal{M}_{\mathrm{B}}$ are different.

\subsection{The proof of Theorem 1.2}

We recommend that at this point the reader reviews the strategy described in Section 2.5.2.

The small quantum cohomology locus $\mathcal{M}_{\mathbb{P}(1,1,1,3)}$ of $\mathbb{P}(1,1,1,3)$ is given by:

$$
\left\{\mathfrak{y}_{1}=0\right\}=\left\{\mathfrak{t}_{1}=0\right\}=\left\{\tau_{1}=0\right\}
$$

Along this locus, the two flat coordinates coincide: $\tau_{2}=\mathfrak{t}_{2}$. As $q_{1}=e^{\tau_{1}}, q_{2}=e^{\tau_{2}}$, and $q=e^{3 t_{2}}$ we have

$$
q_{1}=1, \quad q_{2}=\sqrt[3]{q} .
$$

We calculate the identification (along this locus) between the quantum cohomology algebras of $\mathbb{P}(1,1,1,3)$ and $\mathbb{F}_{3}$ by first finding differential operators which represent our chosen basis for $H_{\text {orb }}^{\bullet}(\mathbb{P}(1,1,1,3) ; \mathbb{C})$ through derivatives of $I_{\mathbb{P}(1,1,1,3)}$, then commuting these operators past the symplectic transformation $\mathbb{U}$ in the equality $\mathbb{U}\left(I_{\mathbb{P}(1,1,1,3)}(y,-z)\right)=I_{\mathbb{F}_{3}}\left(0, y^{1 / 3},-z\right)$, and finally comparing the resulting derivatives of $I_{\mathbb{F}_{3}}$ with our chosen basis for $H^{\bullet}\left(\mathbb{F}_{3} ; \mathbb{C}\right)$. In detail, this goes as follows.

The opposite subspace $\mathcal{H}_{c_{2}}^{-}$at the cusp $c_{2}$ for $\mathbb{P}(1,1,1,3)$ determines a trivialization of $\mathcal{E}$. Define differential operators $P_{i}(z \partial)$ by

$$
\begin{array}{lll}
P_{0}=1, & P_{1}=z \partial, & P_{2}=(z \partial)^{2}, \\
P_{3}=(z \partial)^{3}, & P_{4}=y^{-1 / 3} 3(z \partial)^{4}, & P_{5}=y^{-1 / 3} 3 z \partial\left(y^{-1 / 3} 3(z \partial)^{4}\right)
\end{array}
$$


where $\partial=y \frac{\partial}{\partial y}$. The sections $e_{i}=P_{i}\left(\nabla_{\partial}^{z}\right)\left[\exp \left(W_{y}\right) \omega_{y}\right]$ form a frame of $\mathcal{E}$ which is constant with respect to this trivialization. In fact, from

$$
I_{\mathbb{P}(1,1,1,3)}=y^{p / z}\left(\mathbf{1}_{0}+\frac{27 y^{1 / 3}}{z^{4}} \mathbf{1}_{1 / 3}+\frac{27 y^{2 / 3}}{16 z^{5}} \mathbf{1}_{2 / 3}+\frac{y}{6 z^{6}} \mathbf{1}_{0}+O\left(z^{-7}\right)\right)
$$

one finds that:

$$
\begin{array}{ll}
P_{i} I_{\mathbb{P}(1,1,1,3)}=p^{i}+O\left(z^{-1}\right) & 0 \leq i \leq 3 \\
P_{4} I_{\mathbb{P}(1,1,1,3)}=\mathbf{1}_{1 / 3}+O\left(z^{-1}\right) & \\
P_{5} I_{\mathbb{P}(1,1,1,3)}=\mathbf{1}_{2 / 3}+O\left(z^{-1}\right) &
\end{array}
$$

and therefore that the differential operators $P_{0}, \ldots, P_{5}$ correspond to the basis $1, p$, $p^{2}, p^{3}, \mathbf{1}_{1 / 3}, \mathbf{1}_{2 / 3}$ for the quantum cohomology algebra of $\mathbb{P}(1,1,1,3)$. The matrix of quantum multiplication $p \circ_{q}$ can be obtained as the connection matrix of $\nabla_{\partial}^{z}$ with respect to the frame $\left\{e_{i}\right\}$ :

$$
\nabla_{\partial}^{z}=z \partial+\left(\begin{array}{cccccc}
0 & 0 & 0 & 0 & 0 & \frac{1}{3} y^{1 / 3} \\
1 & 0 & 0 & 0 & 0 & 0 \\
0 & 1 & 0 & 0 & 0 & 0 \\
0 & 0 & 1 & 0 & 0 & 0 \\
0 & 0 & 0 & \frac{1}{3} y^{1 / 3} & 0 & 0 \\
0 & 0 & 0 & 0 & \frac{1}{3} y^{1 / 3} & 0
\end{array}\right)
$$

(Recall that the mirror map for $\mathbb{P}(1,1,1,3)$ is trivial, so $q=y$.)

On the other hand, the sections $e_{i}$ are not constant with respect to the trivialization associated with $\mathcal{H}_{c_{1}}^{-}$. By using $\left.P_{i} I_{\mathbb{F}_{3}}\right|_{\mathfrak{y}_{1}=0}=\overline{\mathbb{U}} P_{i} I_{\mathbb{P}(1,1,1,3)}$ and the expansion (59), one finds that:

$$
\begin{array}{rlr}
\left.P_{i} I_{\mathbb{F}_{3}}\right|_{\mathfrak{y}_{1}=0}=\left(\frac{p_{2}}{3}\right)^{i}+O\left(z^{-1}\right) & 0 \leq i \leq 2 \\
\left.P_{3} I_{\mathbb{F}_{3}}\right|_{\mathfrak{y}_{1}=0}=\frac{p_{2}^{3}}{27}-\sqrt{3} \beta_{1} y^{1 / 3} \mathfrak{p}_{1}+O\left(z^{-1}\right) & \\
\left.P_{4} I_{\mathbb{F}_{3}}\right|_{\mathfrak{y}_{1}=0}=-\sqrt{3} \beta_{1} \mathfrak{p}_{1} z+\frac{\pi}{3} \beta_{1} \mathfrak{p}_{1}^{2}+O\left(z^{-1}\right) & \\
\left.P_{5} I_{\mathbb{F}_{3}}\right|_{\mathfrak{y}_{1}=0}=\sqrt{3} \beta_{2} \mathfrak{p}_{1}+\frac{1}{z}\left(\frac{\pi}{3} \beta_{2} \mathfrak{p}_{1}^{2}+y^{1 / 3}\right)+O\left(z^{-2}\right) &
\end{array}
$$

where $\beta_{i}=2 \pi /\left(9 \Gamma(i / 3)^{3}\right)$. From this, we see that the frame of $\mathcal{E}$ given by

$$
e_{0}, \quad e_{1}, \quad e_{2}, \quad e_{3}, \quad e_{4}+z \frac{\beta_{1}}{\beta_{2}} e_{5}, \quad e_{5}
$$


corresponds to the nonconstant basis

$$
1, \quad \frac{p_{2}}{3}, \quad \frac{p_{2}^{2}}{9}, \quad \frac{p_{2}^{3}}{27}-\sqrt{3} \beta_{1} y^{1 / 3} \mathfrak{p}_{1}, \quad \frac{\beta_{1}}{\beta_{2}} y^{1 / 3}+\frac{2 \pi}{3} \beta_{1} \mathfrak{p}_{1}^{2}, \quad \sqrt{3} \beta_{2} \mathfrak{p}_{1}
$$

for the quantum cohomology algebra of $\mathbb{F}_{3}$. The Dubrovin connection for the quantum cohomology of $\mathbb{F}_{3}$ can be obtained from the connection (60) by the gauge transformation $\Theta(y, z): H_{\text {orb }}^{\bullet}(\mathbb{P}(1,1,1,3)) \rightarrow H^{\bullet}\left(\mathbb{F}_{3}\right)$ given by:

$$
\begin{array}{ll}
\Theta(y, z)\left(p^{i}\right)=\left(\frac{p_{2}}{3}\right)^{i} & 0 \leq i \leq 2 \\
\Theta(y, z)\left(p^{3}\right)=\frac{p_{2}^{3}}{27}-\sqrt{3} \beta_{1} y^{1 / 3} \mathfrak{p}_{1} & \\
\Theta(y, z)\left(\mathbf{1}_{1 / 3}\right)=\frac{\beta_{1}}{\beta_{2}} y^{1 / 3}+\frac{2 \pi}{3} \beta_{1} \mathfrak{p}_{1}^{2}-z \sqrt{3} \beta_{1} \mathfrak{p}_{1} & \\
\Theta(y, z)\left(\mathbf{1}_{2 / 3}\right)=\sqrt{3} \beta_{2} \mathfrak{p}_{1} &
\end{array}
$$

Therefore the quantum product by $p_{2} / 3$ and by $p$, at $q \in \mathcal{M}_{\mathbb{P}(1,1,1,3)}$, are related by conjugation by $\Theta(q):=\Theta(q, 0)$ :

$$
\left.\left(\frac{p_{2}}{3} \circ\left(q_{1}, q_{2}\right)\right)\right|_{\left(q_{1}, q_{2}\right)=(1, \sqrt[3]{q})}=\Theta(q)(p \circ q) \Theta(q)^{-1}
$$

It is easy to check that $\Theta(q)$ preserves the (orbifold) Poincaré pairing and grading. Because $\Theta(q)$ preserves the unit and $p \circ_{q}$ generates the small quantum cohomology algebra, $\Theta(q)$ is an algebra isomorphism. This proves Theorem 1.2.

Remark 3.14 The symplectic transformation $\mathbb{U}$ does not induce an isomorphism between the Frobenius manifolds associated to the big quantum cohomologies of $\mathbb{F}_{3}$ and $\mathbb{P}(1,1,1,3)$ but it does induce an isomorphism between the corresponding $F$-manifolds.

Remark 3.15 The basis change operator $\Theta(q)$ becomes a ring isomorphism because it preserves the unit. If we have a miniversal extended $\mathrm{B}$-model moduli space $\widehat{\mathcal{M}}_{\mathrm{B}}$ corresponding to big quantum cohomology and a mirror $D$-module $\mathcal{E}$ on it-in fact we can reconstruct these from the small data - then the basis change operator $\Theta(q, z)$ between two flat frames can be extended to $q \in \widehat{\mathcal{M}}_{\mathrm{B}}$. The operator $\Theta(q)$ will not necessarily preserve the unit outside the original $\mathrm{B}$-model moduli space $\mathcal{M}_{\mathrm{B}} \subset \widehat{\mathcal{M}}_{\mathrm{B}}$, and so will not in general be a ring isomorphism there. A ring isomorphism over the whole of $\widehat{\mathcal{M}}_{\mathrm{B}}$ is given by $v \longmapsto \Theta(q)\left(v \circ_{q}\right) \Theta(q)^{-1} 1$, but outside of $\mathcal{M}_{\mathrm{B}}$ this will not in general preserve the (orbifold) Poincaré pairing. 


\section{Example: $\mathbb{F}_{2}$ and $\mathbb{P}(1,1,2)$}

We now consider the examples $\mathcal{X}=\mathbb{P}(1,1,2)$ and $Y=\mathbb{F}_{2}$, proving Theorem 1.1 and Conjecture 1.3. The argument is entirely parallel to that in Section 3 and so we omit many details. The only significant difference is in the conclusion which we draw. Since the opposite subspaces associated to the cusps for $\mathbb{P}(1,1,2)$ and $\mathbb{F}_{2}$ agree under parallel transport —or, more concretely, because the symplectic transformation $\mathbb{U}: \mathcal{H}_{\mathbb{P}(1,1,2)} \rightarrow \mathcal{H}_{\mathbb{F}_{2}}$ in Proposition 4.2 maps $\mathcal{H}_{\mathbb{P}(1,1,2)}^{-}$to $\mathcal{H}_{\mathbb{F}_{2}}^{-}$-it follows that the flat structures associated to $\mathbb{P}(1,1,2)$ and $\mathbb{F}_{2}$ agree under analytic continuation. This implies that the big quantum cohomology Frobenius manifolds for $\mathbb{P}(1,1,2)$ and $\mathbb{F}_{2}$ become isomorphic after analytic continuation, and hence that the original form of the Bryan-Graber Conjecture holds.

\subsection{The Landau-Ginzburg mirror to $\mathbb{F}_{2}$}

The surface $\mathbb{F}_{2}$ is a GIT quotient of $\mathbb{C}^{4}$ by $\left(\mathbb{C}^{\times}\right)^{2}$ where $\left(\mathbb{C}^{\times}\right)^{2}$ acts via the inclusion:

$$
\left(\mathbb{C}^{\times}\right)^{2} \hookrightarrow\left(\mathbb{C}^{\times}\right)^{4} \quad(s, t) \mapsto\left(s, s, s^{-2} t, t\right)
$$

The mirror family $\pi: Z \rightarrow \mathcal{M}_{\mathbb{F}_{2}}$ is given by restricting the dual of this inclusion

$$
\begin{aligned}
\pi:\left(\mathbb{C}^{\times}\right)^{4} & \longrightarrow\left(\mathbb{C}^{\times}\right)^{2} \\
\left(w_{1}, \ldots, w_{4}\right) & \longmapsto\left(w_{1} w_{2} w_{3}^{-2}, w_{3} w_{4}\right)
\end{aligned}
$$

to the open subset $\mathcal{M}_{\mathbb{F}_{2}} \subset\left(\mathbb{C}^{\times}\right)^{2}$ defined by:

$$
\mathcal{M}_{\mathbb{F}_{2}}=\left\{\left(y_{1}, y_{2}\right) \in\left(\mathbb{C}^{\times}\right)^{2}: y_{1} \neq \frac{1}{4}\right\}
$$

The superpotential $W$ is

$$
W=w_{1}+w_{2}+w_{3}+w_{4}
$$

and the holomorphic volume form $\omega_{y}$ on the fiber $Z_{y}=\pi^{-1}\left(y_{1}, y_{2}\right)$ is:

$$
\omega_{y}=\frac{d \log w_{1} \wedge \cdots \wedge d \log w_{4}}{d \log y_{1} \wedge d \log y_{2}}
$$


We deleted the locus $y_{1}=\frac{1}{4}$ from $\mathcal{M}_{\mathbb{F}_{2}}$ to ensure that Assumptions 2.17 hold. The oscillating integrals (34) here form a basis of solutions to the Picard-Fuchs equations:

$$
\begin{aligned}
D_{2}\left(D_{2}-2 D_{1}\right) f & =y_{2} f \\
D_{1}^{2} D_{2}\left(D_{2}-z\right) f & =y_{1} y_{2}^{2} f \\
D_{1}^{2} D_{2} f & =y_{1} y_{2}\left(D_{2}-2 D_{1}\right) f \\
D_{1}^{2} f & =y_{1}\left(D_{2}-2 D_{1}\right)\left(D_{2}-2 D_{1}-z\right) f
\end{aligned}
$$

where $D_{1}=z y_{1} \frac{\partial}{\partial y_{1}}$ and $D_{2}=z y_{2} \frac{\partial}{\partial y_{2}}$.

\subsection{Mirror symmetry for $\mathbb{F}_{2}$}

Givental's mirror theorem [26, Theorem 0.1] implies that the $I$-function

(62) $I_{\mathbb{F}_{2}}\left(y_{1}, y_{2}, z\right)$

$$
=z \sum_{k, l \geq 0} \frac{y_{1}^{k+p_{1} / z} y_{2}^{l+p_{2} / z} \prod_{m=-\infty}^{0}\left(p_{2}-2 p_{1}+m z\right)}{\prod_{m=1}^{k}\left(p_{1}+m z\right)^{2} \prod_{m=1}^{l}\left(p_{2}+m z\right) \prod_{m=-\infty}^{l-2 k}\left(p_{2}-2 p_{1}+m z\right)}
$$

where $p_{1}, p_{2}$ is the basis of $H^{2}\left(\mathbb{F}_{2}\right)$ defined in Section 2.1.1, coincides with the small $J$-function (30) after a change of variables $\left(y_{1}, y_{2}\right) \mapsto\left(q_{1}, q_{2}\right)$ :

$$
J_{\mathbb{F}_{2}}\left(q_{1}, q_{2}, z\right)=I_{\mathbb{F}_{2}}\left(y_{1}, y_{2}, z\right)
$$

The components of $I_{\mathbb{F}_{2}}\left(y_{1}, y_{2}, z\right)$ form another basis of solutions to the Picard-Fuchs system (61). As we explained in Section 2.5.2, this proves Conjecture 2.21 for $\mathbb{F}_{2}$.

As before, we can read off the mirror map $\left(y_{1}, y_{2}\right) \mapsto\left(q_{1}, q_{2}\right)$ by expanding the $I$-function as a Laurent series in $z^{-1}$. This gives:

$$
\begin{aligned}
& q_{1}=y_{1} \exp \left(2 \sum_{k \geq 1} \frac{(2 k-1) !}{(k !)^{2}} y_{1}^{k}\right)=\frac{4 y_{1}}{\left(1+\sqrt{1-4 y_{1}}\right)^{2}} \\
& q_{2}=y_{2} \exp \left(-\sum_{k \geq 1} \frac{(2 k-1) !}{(k !)^{2}} y_{1}^{k}\right)=\frac{y_{2}\left(1+\sqrt{1-4 y_{1}}\right)}{2}
\end{aligned}
$$

The mirror map identifies a neighbourhood of $y_{1}=y_{2}=0$ in $\mathcal{M}_{\mathbb{F}_{2}}$ with the Kähler moduli space $\mathcal{M}_{\mathrm{A}}$ of $\mathbb{F}_{2}$; here once again $\left(q_{1}, q_{2}\right)$ are coordinates on $\mathcal{M}_{\mathrm{A}}$. This identification matches up the Jacobi ring $J\left(W_{y}\right)$ of $W_{y}$ with the small quantum cohomology algebra of $\mathbb{F}_{2}$ at $\left(q_{1}, q_{2}\right)$ and the residue pairing with the Poincaré pairing. 


\subsection{The Landau-Ginzburg mirror to $\mathbb{P}(1,1,2)$}

The mirror family $\pi$ is

$$
\begin{aligned}
\pi: Z=\left(\mathbb{C}^{\times}\right)^{3} & \longrightarrow \mathcal{M}_{\mathbb{P}(1,1,2)}=\left(\mathbb{C}^{\times}\right) \\
\left(w_{1}, w_{2}, w_{4}\right) & \longmapsto w_{1} w_{2} w_{4}^{2}
\end{aligned}
$$

the superpotential $W$ is

$$
W=w_{1}+w_{2}+w_{4}
$$

and the holomorphic volume form $\omega_{y}$ on the fiber $Z_{y}=\pi^{-1}(y)$ is:

$$
\omega_{y}=\frac{d \log w_{1} \wedge d \log w_{2} \wedge d \log w_{4}}{d \log y}
$$

The oscillating integrals (34) satisfy the Picard-Fuchs equation

$$
D^{2}(2 D)(2 D-z) f=y f .
$$

where $D=z y \frac{\partial}{\partial y}$.

\subsection{Mirror symmetry for $\mathbb{P}(1,1,2)$}

A theorem of Coates-Corti-Lee-Tseng [15, Theorem 1.7] shows that the $I$-function

$$
I_{\mathbb{P}(1,1,2)}(y, z)=z y^{p / z} \sum_{\substack{d: 2 d \in \mathbb{Z} \\ d \geq 0}} \frac{y^{d}}{\prod_{\substack{b:\langle b\rangle=\langle d\rangle \\ 0<b \leq d}}(p+b z)^{2} \prod_{\substack{b:\langle b\rangle=0 \\ 0<b \leq 2 d}}(2 p+b z)} \mathbf{1}_{\langle d\rangle}
$$

coincides with the small $J$-function (29) after the (trivial) change of variables $q=y$ :

$$
J_{\mathbb{P}(1,1,2)}(q, z)=I_{\mathbb{P}(1,1,2)}(y, z)
$$

The components of $I_{\mathbb{P}(1,1,2)}(y, z)$ give another basis of solutions to the Picard-Fuchs equation (63). As before this proves Conjecture 2.21 for $\mathbb{P}(1,1,2)$. The moduli space $\mathcal{M}_{\mathbb{P}(1,1,2)}$ with coordinate $y$ is identified via the map $q=y$ with the Kähler moduli space $\mathcal{M}_{\mathrm{A}}$ of $\mathbb{P}(1,1,2)$; here $q$ is again a coordinate on $\mathcal{M}_{\mathrm{A}}$. This identification matches the Jacobi ring $J\left(W_{q}\right)$ with the small quantum orbifold cohomology algebra of $\mathbb{P}(1,1,2)$ at $q$, and the residue pairing with the Poincaré pairing.

\subsection{Constructing the B-model VSHS}

As in Section 3.5, we extend the Landau-Ginzburg mirror of $\mathbb{F}_{2}$ to a Landau-Ginzburg model with a larger base defined in terms of the secondary fan for $\mathbb{F}_{2}$ (Figure 4). 


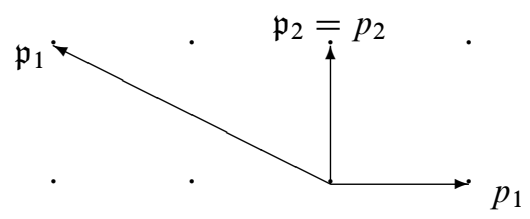

Figure 4: The secondary fan for $\mathbb{F}_{2}$

Take $w_{1}, w_{4}$ as coordinates on the fiber $Z_{y}$, so that:

$$
W_{y}=w_{1}+\frac{y_{1} y_{2}^{2}}{w_{1} w_{4}^{2}}+\frac{y_{2}}{w_{4}}+w_{4}
$$

Let $\overline{\mathcal{M}}$ be the toric orbifold associated to the secondary fan for $\mathbb{F}_{2}$. There are two distinguished coordinate patches on $\overline{\mathcal{M}}$, one for each maximal cone in the secondary fan. Let $\left(y_{1}, y_{2}\right)$ be the coordinates dual to $\left(p_{1}, p_{2}\right)$ and $\left(\mathfrak{y}_{1}, \mathfrak{y}_{2}\right)$ be the coordinates dual to $\left(\mathfrak{p}_{1}, \mathfrak{p}_{2}\right)$ (see Figure 4 ). As

$$
\mathfrak{y}_{1}=y_{1}^{-1 / 2} \quad \mathfrak{y}_{2}=y_{1}^{1 / 2} y_{2}
$$

we see that $\left(\mathfrak{y}_{1}, \mathfrak{y}_{2}\right)$ is a uniformizing system near a $\mathbb{Z} / 2 \mathbb{Z}$ orbifold point at $\left(\mathfrak{y}_{1}, \mathfrak{y}_{2}\right)=0$. In the coordinates $\left(\mathfrak{y}_{1}, \mathfrak{y}_{2}\right)$ we have

$$
W_{y}=w_{1}+\frac{\mathfrak{y}_{2}^{2}}{w_{1} w_{4}^{2}}+\frac{\mathfrak{y}_{1} \mathfrak{y}_{2}}{w_{4}}+w_{4}
$$

and so we can extend the family of tori $\pi$ and the superpotential $W_{y}$ across the locus $\left\{\mathfrak{y}_{1}=0\right\}$. Here we see

$$
W=w_{1}+w_{2}+w_{4}, \quad w_{1} w_{2} w_{4}^{2}=\mathfrak{y}_{2}^{2}
$$

which is the Landau-Ginzburg mirror to $\mathbb{P}(1,1,2)$ : the locus $\mathfrak{y}_{1}=0, \mathfrak{y}_{2} \neq 0$ in $\overline{\mathcal{M}}$ is identified with $\mathcal{M}_{\mathbb{P}(1,1,2)}$ via the map $y=\mathfrak{y}_{2}^{2}$.

The $B$-model moduli space $\mathcal{M}_{\mathrm{B}}$ here, which is the base of our extended LandauGinzburg model, is obtained from $\overline{\mathcal{M}}$ by deleting the closures of the loci $\left\{y_{1} y_{2}=0\right\}$ and $\left\{y_{1}=1 / 4\right\}$. Equations (65) and (66) together define a Landau-Ginzburg model over $\mathcal{M}_{\mathrm{B}}$ which contains the Landau-Ginzburg mirrors for $\mathbb{F}_{2}$ and for $\mathbb{P}(1,1,2)$ as subsets. The limit points $y_{1}=y_{2}=0$ and $\mathfrak{y}_{1}=\mathfrak{y}_{2}=0$ of $\mathcal{M}_{\mathrm{B}}$ are called the large radius limit points or cusps corresponding respectively to $\mathbb{F}_{2}$ and to $\mathbb{P}(1,1,2)$. Let $\mathcal{E}$ denote the B-model VSHS with base $\mathcal{M}_{\mathrm{B}}$ defined by the Landau-Ginzburg model 
just described and equipped with the grading operator:

$$
\operatorname{Gr}\left[f(x, z) e^{W_{y} / z} \omega_{y}\right]=\left[\left(2 z \partial_{z}+2 \sum_{i=1}^{4} w_{i} \partial_{w_{i}}\right) f(x, z) e^{W_{y} / z} \omega_{y}\right]
$$

The Euler field and dimension here are

$$
E=2 y_{2} \frac{\partial}{\partial y_{2}}=2 \mathfrak{y}_{2} \frac{\partial}{\partial \mathfrak{y}_{2}}, \quad D=\operatorname{dim} \mathbb{F}_{2}=2 .
$$

Remark 4.1 The superpotentials (65) and (66) have isolated nondegenerate critical points; this implies that the small quantum cohomology algebra of $\mathbb{F}_{2}$ and the small quantum orbifold cohomology algebra of $\mathbb{P}(1,1,2)$ are semisimple.

\subsection{An opposite subspace at each cusp}

As before, the $I$-functions (62), (64) and the isomorphism (36) define $D$-module homomorphisms:

$$
\begin{aligned}
& \mathbb{I}_{\mathbb{F}_{2}}: \mathcal{E} \longrightarrow H^{\bullet}\left(\mathbb{F}_{2}\right) \otimes \mathcal{O}_{\widetilde{\mathcal{M}}_{\mathrm{B}}}\left\{z, z^{-1}\right\}, \\
& \mathbb{I}_{\mathbb{P}(1,1,2)}:\left.\mathcal{E}\right|_{\mathcal{M}_{\mathbb{P}(1,1,2)}} \longrightarrow H_{\text {orb }}^{\bullet}(\mathbb{P}(1,1,2)) \otimes \mathcal{O}_{\widetilde{\mathcal{M}}_{\mathbb{P}(1,1,2)}}\left\{z, z^{-1}\right\}
\end{aligned}
$$

by sending $P(y, z \partial, z) \in \mathcal{E} \cong \mathcal{O}_{\mathcal{M}_{\mathrm{B}}}\{z\}\left\langle z \mathcal{T}_{\mathcal{M}_{\mathrm{B}}}\right\rangle$ to $z^{-1} P I_{\mathbb{F}_{2}}$ or to $z^{-1} P I_{\mathbb{P}(1,1,2)}$. The maps (67) send $\nabla^{z}$-parallel sections of $\mathcal{E}$ to constant sections, and thus identify the space $\mathcal{H}$ of flat sections of $\mathcal{E}$ with Givental's symplectic vector space:

$$
\begin{aligned}
\mathbb{I}_{\mathbb{F}_{2}}: \mathcal{H} & \cong H^{\bullet}\left(\mathbb{F}_{2}\right) \otimes \mathbb{C}\left\{z, z^{-1}\right\}=\left.\mathcal{H}_{\mathbb{F}_{2}}\right|_{Q_{i}=1} \\
\mathbb{I}_{\mathbb{P}(1,1,2)}: \mathcal{H} & \cong H_{\text {orb }}^{\bullet}(\mathbb{P}(1,1,2)) \otimes \mathbb{C}\left\{z, z^{-1}\right\}=\left.\mathcal{H}_{\mathbb{P}(1,1,2)}\right|_{Q=1}
\end{aligned}
$$

Here $\mathbb{I}_{\mathbb{F}_{2}}$, which is a priori defined only in a small neighbourhood of the cusp for $\mathbb{F}_{2}$, is extended to the whole of $\widetilde{\mathcal{M}}_{\mathrm{B}}$ by analytic continuation.

Let $c_{1}$ and $c_{2}$ denote the cusps of $\mathcal{M}_{\mathrm{B}}$ corresponding respectively to $\mathbb{F}_{2}$ and $\mathbb{P}(1,1,2)$. We define opposite subspaces $\mathcal{H}_{c_{1}}^{-}$and $\mathcal{H}_{c_{2}}^{-}$of $\mathcal{H}$ by:

$$
\mathbb{I}_{\mathbb{F}_{2}}\left(\mathcal{H}_{c_{1}}^{-}\right)=\left.\mathcal{H}_{\mathbb{F}_{2}}^{-}\right|_{Q_{i}=1} \quad \mathbb{I}_{\mathbb{P}(1,1,2)}\left(\mathcal{H}_{c_{2}}^{-}\right)=\left.\mathcal{H}_{\mathbb{P}(1,1,2)}^{-}\right|_{Q=1}
$$

These opposite subspaces are uniquely characterized by monodromy and homogeneity properties, as in Theorem 3.5, but we will not pursue this here. 


\subsection{The polarizations match}

Define $\overline{\mathbb{U}}:\left.\left.\mathcal{H}_{\mathbb{P}(1,1,2)}\right|_{Q=1} \rightarrow \mathcal{H}_{\mathbb{F}_{2}}\right|_{Q_{i}=1}$ to be the composition

$$
\left.\left.\mathcal{H}_{\mathbb{P}(1,1,2)}\right|_{Q=1} \stackrel{\left(\mathbb{I}_{\mathbb{P}(1,1,2)}\right)^{-1}}{\longrightarrow} \mathcal{H} \stackrel{\mathbb{I}_{\mathbb{F}_{2}}}{\longrightarrow} \mathcal{H}_{\mathbb{F}_{2}}\right|_{Q_{i}=1}
$$

and let $\mathbb{U}:\left.\left.\mathcal{H}_{\mathbb{P}(1,1,2)}\right|_{Q=1} \rightarrow \mathcal{H}_{\mathbb{F}_{2}}\right|_{Q_{i}=1}$ be $\overline{\mathbb{U}}$ followed by changing the sign of $z$. Arguing as in the proof of Proposition 3.9 shows:

Proposition 4.2 The matrix of $\mathbb{U}$ with respect to the bases for $H_{\mathrm{orb}}^{\bullet}(\mathbb{P}(1,1,2))$ and $H^{\bullet}\left(\mathbb{F}_{2}\right)$ defined in Section 2.1.1 is:

$$
\left(\begin{array}{cccc}
1 & 0 & 0 & 0 \\
\frac{\pi \mathrm{i}}{z} & 0 & 0 & i \\
-\frac{\pi \mathrm{i}}{2 z} & \frac{1}{2} & 0 & -\frac{i}{2} \\
\frac{\pi^{2}}{4 z^{2}} & 0 & \frac{1}{2} & \frac{\pi}{2 z}
\end{array}\right)
$$

The linear transformation $\mathbb{U}$ preserves the grading, the symplectic forms, and the standard opposite subspaces.

Note that here $\mathbb{U}$ takes the form $\left.\exp \left(-\pi i\left(p_{2}-2 p_{1}\right) /(2 z)\right) \circ \mathbb{U}\right|_{z=\infty}$.

\subsection{The proof of Conjecture 1.3}

By applying Dubrovin's Reconstruction Theorem, as in the proof of Theorem 3.12, we deduce Conjectures 1.3 and 5.1 for $\mathcal{X}=\mathbb{P}(1,1,2)$ and $Y=\mathbb{F}_{2}$ :

Theorem 4.3 Let $\left.\mathbb{E}_{\tau}^{\mathbb{F}_{2}} \subset \mathcal{H}_{\mathbb{F}_{2}}\right|_{Q_{i}=1}$ and $\left.\mathbb{E}_{\tau}^{\mathbb{P}(1,1,2)} \subset \mathcal{H}_{\mathbb{P}(1,1,2)}\right|_{Q=1}$ be the moving subspace realizations (25) of the analytic big A-model VSHSs of $\mathbb{F}_{2}$ and $\mathbb{P}(1,1,2)$. Then there exists a map $\Upsilon$ from an open subset of $H_{\mathrm{orb}}^{\bullet}(\mathbb{P}(1,1,2))$ to an open subset of $H^{\bullet}\left(\mathbb{F}_{2}\right)$ such that, after analytic continuation if necessary,

$$
\overline{\mathbb{U}}\left(\mathbb{E}_{\tau}^{\mathbb{P}(1,1,2)}\right)=\mathbb{E}_{\Upsilon(\tau)}^{\mathbb{F}_{2}}
$$

where $\mathbb{U}$ is the symplectic transformation from Proposition 4.2.

\subsection{The proof of Theorem 1.1}

It remains only to prove Theorem 1.1. But since the transformation $\mathbb{U}$ maps:

- the big A-model VSHS for $\mathbb{P}(1,1,2)$ to the big A-model VSHS for $\mathbb{F}_{2}$ 
- the standard opposite subspace $\mathcal{H}_{\mathbb{P}(1,1,2)}^{-}$to the standard opposite subspace $\mathcal{H}_{\mathbb{F}_{2}}^{-}$

- the dilaton shift $1 \in z \mathcal{H}_{\mathbb{P}(1,1,2)}^{-} / \mathcal{H}_{\mathbb{P}(1,1,2)}^{-}$to the dilaton shift $1 \in z \mathcal{H}_{\mathbb{F}_{2}}^{-} / \mathcal{H}_{\mathbb{F}_{2}}^{-}$

it follows immediately that $\mathbb{U}$ induces an isomorphism between the big quantum cohomology Frobenius manifolds associated to $\mathcal{X}=\mathbb{P}(1,1,2)$ and $Y=\mathbb{F}_{2}$. To compute this isomorphism explicitly, consider the discussion before Proposition 2.12. The underlying linear isomorphism $\Theta: H_{\text {orb }}^{\bullet}(\mathcal{X} ; \mathbb{C}) \rightarrow H^{\bullet}(Y ; \mathbb{C})$ here is the isomorphism $z \mathcal{H}_{\mathcal{X}}^{-} / \mathcal{H}_{\mathcal{X}}^{-} \cong z \mathcal{H}_{Y}^{-} / \mathcal{H}_{Y}^{-}$induced by $\mathbb{U}$, so $\Theta=\left.\mathbb{U}\right|_{z=\infty}$ :

$$
\begin{array}{ll}
\Theta\left(\mathbf{1}_{0}\right)=1 & \Theta(p)=\frac{p_{2}}{2} \\
\Theta\left(p^{2}\right)=\left(\frac{p_{2}}{2}\right)^{2} & \Theta\left(\mathbf{1}_{1 / 2}\right)=-\frac{i}{2}\left(p_{2}-2 p_{1}\right)
\end{array}
$$

The map $\Theta$ evidently preserves the Poincaré pairings. It gives an isomorphism of algebras between

$$
\left(H_{\text {orb }}^{\bullet}(\mathcal{X} ; \mathbb{C}), \bullet_{\tau}\right) \quad \text { and } \quad\left(H^{\bullet}(Y ; \mathbb{C}), \bullet_{f(\tau)}\right)
$$

where we can read off the affine-linear identification of flat coordinates $\tau \mapsto f(\tau)$ from the big $J$-functions: $\mathbb{U}\left(J_{\mathcal{X}}(\tau,-z)\right)=J_{Y}(f(\tau),-z)$, and so

$$
f(\tau)=\Theta(\tau)+\frac{\pi i}{2}\left(p_{2}-2 p_{1}\right) .
$$

Here we are considering the analytic version of Givental's formalism, with the Novikov variables $Q, Q_{1}$, and $Q_{2}$ set to 1 . Putting back the Novikov variables using the Divisor Equation (see Remark 2.16 above) one finds that one can absorb the shift of the origin in (70) into the specialization of quantum parameters:

$$
Q_{1}=-1, \quad Q_{2}=\mathrm{i} Q^{1 / 2} .
$$

Theorem 1.1 is proved.

\section{A Crepant Resolution Conjecture}

In this final section we formulate our version of the Crepant Resolution Conjecture. This is a more precise version of Conjecture 1.3 from the Introduction. We discuss its relationship with theorems of Lupercio-Poddar and Yasuda, and show that under a Hard Lefschetz condition it implies the original form of the Bryan-Graber Conjecture. We also indicate several aspects of the story which remain to be explored.

Conjecture 5.1 Let $\mathcal{X}$ be an orbifold with projective coarse moduli space $X$ and let $\pi: Y \rightarrow X$ be a crepant resolution. Suppose that the big quantum products $\bullet_{\tau}$ in (5) 
for $\mathcal{X}$ and $Y$ are convergent as functions of $\tau$ and $Q$, so that the analytic big $A$-model VSHS of $\mathcal{X}$ (respectively of $Y$ ) with Novikov variables specialized to 1 is well-defined over an open subset of $H_{\text {orb }}^{\bullet}(\mathcal{X} ; \mathbb{C})$ (respectively of $H^{\bullet}(Y ; \mathbb{C})$ ); see Section 2.3.4.

Let $\left.\mathbb{E}_{\tau}^{\mathcal{X}} \subset \mathcal{H}_{\mathcal{X}}\right|_{Q_{i}=1},\left.\mathbb{E}_{\tau}^{Y} \subset \mathcal{H}_{Y}\right|_{Q_{i}=1}$ be the moving subspace realizations (25) of the analytic big A-model VSHSs of $\mathcal{X}$ and $Y$ respectively. Define the limiting Hodge structure $\mathbb{E}_{\lim , \tau}^{\mathcal{X}}$ associated to $\tau \in H_{\text {orb }}^{\bullet}(\mathcal{X} ; \mathbb{C})$ by:

$$
\mathbb{E}_{\lim , \tau}^{\mathcal{X}}=\lim _{\sigma \rightarrow \text { l.r.l. }} e^{-\sigma / z} \mathbb{E}_{\tau+\sigma}^{\mathcal{X}}
$$

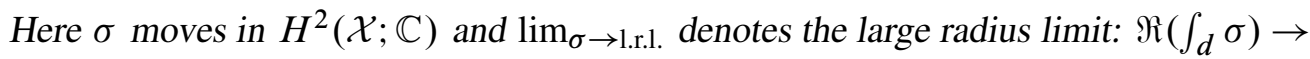
$-\infty$ for all nonzero $d \in \operatorname{Eff}(\mathcal{X})$.

There is a symplectic transformation $\overline{\mathbb{U}}:\left.\left.\mathcal{H}_{\mathcal{X}}\right|_{Q_{i}=1} \rightarrow \mathcal{H}_{Y}\right|_{Q_{i}=1}$ and a map $\Upsilon$ from an open subset of $H_{\text {orb }}^{\bullet}(\mathcal{X} ; \mathbb{C})$ to an open subset of $H^{\bullet}(Y ; \mathbb{C})$ such that, after analytic continuation if necessary,

$$
\overline{\mathbb{U}}\left(\mathbb{E}_{\tau}^{\mathcal{X}}\right)=\mathbb{E}_{\Upsilon(\tau)}^{Y}
$$

and that:

(a) $\overline{\mathbb{U}}$ is degree-preserving and $\mathbb{C}\left\{z, z^{-1}\right\}$-linear.

(b) $\overline{\mathbb{U}}(\rho \cup)=\left(\pi^{\star}(\rho) \cup\right) \overline{\mathbb{U}}$ for all nontwisted degree-two cohomology classes $\rho \in$ $H^{2}(\mathcal{X} ; \mathbb{C})$; here the product on the left-hand side is the Chen-Ruan orbifold cup product and the product on the right-hand side is the usual cup product.

(c) There is a point $\tau_{0} \in H_{\text {orb }}^{2}(\mathcal{X} ; \mathbb{C})$ such that the standard opposite subspaces $\left.\mathcal{H}_{\mathcal{X}}^{-}\right|_{Q_{i}=1}$ and $\left.\mathcal{H}_{Y}^{-}\right|_{Q_{i}=1}$ are opposite to $\mathbb{E}_{\lim , \tau_{0}}^{\mathcal{X}}$ and to $\overline{\mathbb{U}}\left(\mathbb{E}_{\lim , \tau_{0}}^{\mathcal{X}}\right)$ respectively.

Let $\mathbb{U}:\left.\left.\mathcal{H}_{\mathcal{X}}\right|_{Q_{i}=1} \rightarrow \mathcal{H}_{Y}\right|_{Q_{i}=1}$ be $\overline{\mathbb{U}}$ followed by changing the sign of $z$. Conjecture 5.1 and the definition (26) of the analytic version of Givental's cone immediately imply Conjecture 1.3 from the Introduction:

$$
\mathbb{U}\left(\mathcal{L}_{\mathcal{X}}^{\text {an }}\right)=\mathcal{L}_{Y}^{\text {an }}
$$

Note that $\mathbb{U}$ determines the map $\Upsilon$ uniquely (see (23)):

$$
\overline{\mathbb{U}}\left(\mathbb{E}_{\tau}^{\mathcal{X}}\right) \cap\left(1+\left.\mathcal{H}_{Y}^{-}\right|_{Q_{i}=1}\right)=\left\{1+\Upsilon(\tau) / z+O\left(1 / z^{2}\right)\right\} .
$$

In terms of the Lagrangian cones, the base space of the big A-model VSHS arises as a space parametrizing tangent spaces to the cone $\mathcal{L}_{\mathcal{X}}^{\text {an }}$ or $\mathcal{L}_{Y}^{\text {an }}$. From this viewpoint, the map $\Upsilon$ can be interpreted as the map between the moduli spaces of tangent spaces to the cones induced from the isomorphism $\mathbb{U}: \mathcal{L}_{\mathcal{X}}^{\mathrm{an}} \cong \mathcal{L}_{Y}^{\mathrm{an}}$. 
Remark 5.2 Even though the Gromov-Witten theories of $\mathcal{X}$ and $Y$ are defined over $\mathbb{Q}$, the transformation $\mathbb{U}$ may only be defined over $\mathbb{C}$. This happened here for both $\mathcal{X}=\mathbb{P}(1,1,2)$ and $\mathcal{X}=\mathbb{P}(1,1,1,3)$ : see Propositions 4.2 and 3.9.

Remark 5.3 The operator $\mathbb{U}$ will be far from unique because of various degreepreserving symmetries of the Lagrangian cones. The ambiguity of $\mathbb{U}$ by scalar multiplication (dilation symmetry) can be fixed by the condition $\mathbb{U}(1)=1+O\left(z^{-1}\right)$, but there will also be discrete symmetries coming from monodromy of the mirror VSHS. The Divisor Equation implies that

$$
\left.J_{\mathcal{X}}(\tau+\sigma,-z)\right|_{Q=1}=\left.e^{-\sigma / z} J_{\mathcal{X}}(\tau,-z)\right|_{Q^{d_{\mapsto}} e^{\int_{d}} \sigma} \quad \sigma \in H^{2}(\mathcal{X} ; \mathbb{C})
$$

and in particular setting $\sigma=2 \pi i \rho$, where $\rho \in H^{2}(X, \mathbb{Z})$ is an integral degree-two class coming from the coarse moduli space $X$, shows that multiplication by $\exp (2 \pi i \rho / z)$ preserves $\mathcal{L}_{\mathcal{X}}$. This symmetry comes from a monodromy around the large radius limit point. Part (b) can therefore be understood as a compatibility between the monodromy actions on $\mathcal{H}_{\mathcal{X}}$ and $\mathcal{H}_{Y}$. There will also be discrete symmetries of other types; see the discussion in Section 5.5 below.

Remark 5.4 When $\tau \in H_{\text {orb }}^{\bullet}(\mathcal{X} ; \mathbb{C})$ is sufficiently close to the large radius limit, the limiting Hodge structure $\mathbb{E}_{\lim , \tau}^{\mathcal{X}}$ exists and is calculated as:

$$
\mathbb{E}_{\lim , \tau}^{\mathcal{X}}=\lim _{\sigma \rightarrow \text { l.r.l. }} e^{-\sigma / z} \mathbb{E}_{\tau+\sigma}^{\mathcal{X}}=\operatorname{Span}_{\mathbb{C}\{z\}}\left\langle\left.\frac{\partial J_{\mathcal{X}}}{\partial \tau^{\alpha}}(\tau, z)\right|_{Q_{i}=0}\right\rangle_{1 \leq \alpha \leq N}
$$

Part (c) implies that in a neighbourhood of the large radius $\operatorname{limit}$ " $\lim _{\sigma \rightarrow \text { l.r.l. }}\left(\tau_{0}+\sigma\right)$ ", the Frobenius structures associated to both $\mathcal{X}$ and $Y$ are well-defined.

Remark 5.5 We can restore the Novikov variables in equation (71) as follows. Given the analytic Givental cone $\mathcal{L}^{\text {an }}$, we can define a multivalued family of analytic cones $\mathcal{L}_{Q}^{\text {an }}$ parametrized by $r$ complex numbers $Q_{1}, \ldots, Q_{r} \in \mathbb{C}^{\times}$:

$$
\mathcal{L}_{Q}^{\mathrm{an}}:=\exp \left(\sum_{i=1}^{r} p_{i} \log Q_{i} / z\right) \mathcal{L}^{\mathrm{an}}
$$

Due to the discrete symmetries from Remark 5.3, $\mathcal{L}_{Q}^{\text {an }}$ depends only on suitable roots $Q_{1}^{1 / m_{1}}, \ldots, Q_{r}^{1 / m_{r}}$ of the Novikov variables. From Equation (72), the original Givental cone over the Novikov ring $\Lambda$ can be interpreted as the completion of the family of cones $\left\{\mathcal{L}_{Q^{\xi}}^{\text {an }}{ }_{Q \in\left(\mathbb{C}^{\times}\right)^{r}}\right.$ at the origin $Q=0$. By using (b), we have a family version of (71):

$$
\mathbb{U}\left(\mathcal{L}_{\mathcal{X}, Q}^{\mathrm{an}}\right)=\mathcal{L}_{Y, \pi^{*} Q}^{\mathrm{an}}
$$


where $\pi^{*}$ denotes the map between Novikov variables induced by the pullback $\pi^{*}: H^{2}(X) \rightarrow H^{2}(Y)$.

Remark 5.6 The method by which Conjecture 5.1 was proved here (see Sections 3 and 4) is applicable to a broad class of examples. Every time we know a mirror for small quantum cohomology we should have a similar explanation for wall-crossing phenomena in genus-zero Gromov-Witten theory. This has been emphasized in work of Coates [13] and Iritani [38], which provides evidence that something very like Conjecture 5.1 may also hold for more general crepant birational transformations.

Remark 5.7 Conjecture 5.1 is in keeping with ideas of Ruan [46]: that the Lagrangian cones which encode genus-zero Gromov-Witten invariants of an orbifold $\mathcal{X}$ and its crepant resolution $Y$ should coincide after a symplectic transformation, and that the total descendant potentials of $\mathcal{X}$ and of $Y$ (which are generating functions encoding Gromov-Witten invariants of all genera) should be related by the quantization of this symplectic transformation. These ideas were inspired by results of Givental [28], who has found in a number of examples that operations in Gromov-Witten theory which in genus zero give a symplectic transformation of the Lagrangian cone act on higher-genus invariants by applying the quantization of that symplectic transformation (which is a differential operator) to the total descendant potential.

\subsection{Consequences of Conjecture 5.1}

When Conjecture 5.1 holds we can distinguish two cases:

(i) $\mathbb{U}\left(\mathcal{H}_{\mathcal{X}}^{-}\right)=\mathcal{H}_{Y}^{-}$

(ii) $\mathbb{U}\left(\mathcal{H}_{\overline{\mathcal{X}}}^{-}\right) \neq \mathcal{H}_{Y}^{-}$

In case (i), which occurs when matrix elements of $\mathbb{U}$ do not contain positive powers of $z$, the big quantum cohomology Frobenius structures of $\mathcal{X}$ and $Y$ are related by analytic continuation-exactly as in Section 4.9. In this case $\mathbb{U}$ can be thought of as the well-known ambiguity of fundamental solutions in the theory of Frobenius manifolds. In case (ii), which occurs when some matrix elements of $\mathbb{U}$ contain strictly positive powers of $z, \mathbb{U}$ does not preserve the opposite subspaces and the Frobenius manifolds associated to $\mathcal{X}$ and $Y$ will in general be different. In this case $H_{\text {orb }}^{\bullet}(\mathcal{X} ; \mathbb{C})$ and $H^{\bullet}(Y ; \mathbb{C})$ carry the same $F$-manifold structure but have different flat coordinate systems. Note that case (i) happened for $\mathcal{X}=\mathbb{P}(1,1,2)$ and that case (ii) happened for $\mathcal{X}=\mathbb{P}(1,1,1,3)$.

In the next two sections we will show that if $\mathcal{X}$ satisfies a Hard Lefschetz condition then case (ii) cannot occur, and thus that our Conjecture implies the Bryan-Graber Conjecture. 


\subsection{Conjecture 5.1 and theorems of Lupercio-Poddar and Yasuda}

Theorem 5.8 If Conjecture 5.1 holds then $H_{\text {orb }}^{\bullet}(\mathcal{X} ; \mathbb{C})$ and $H^{\bullet}(Y ; \mathbb{C})$ are isomorphic as graded vector spaces.

Remark 5.9 Lupercio-Poddar and Yasuda have shown that if $\mathcal{X}$ and $Y$ are $K-$ equivalent orbifolds then $H_{\text {orb }}^{\bullet}(\mathcal{X} ; \mathbb{C})$ and $H^{\bullet}(Y ; \mathbb{C})$ have the same Hodge numbers $[51 ; 42]$. Thus this consequence itself is not surprising. We include a proof only because the isomorphism which we construct depends ${ }^{16}$ on the choice of $\tau_{0}$ in Conjecture 5.1(c), and so from the point of view of Gromov-Witten theory there may be no distinguished graded isomorphism between $H_{\text {orb }}^{\bullet}(\mathcal{X} ; \mathbb{C})$ and $H^{\bullet}(Y ; \mathbb{C})$.

Proof of Theorem 5.8 Let $\tau_{0} \in H_{\text {orb }}^{2}(\mathcal{X})$ be as in Conjecture 5.1(c). Equation (73) shows that the limiting Hodge structure $\mathbb{E}_{\lim , \tau_{0}}^{\mathcal{X}}$ is spanned over $\mathbb{C}\{z\}$ by homogeneous elements of $\left.\mathcal{H}_{\mathcal{X}}\right|_{Q_{i}=1}$, and hence that $\mathbb{E}_{\text {lim, } \tau_{0}}^{\mathcal{X}}$ is a homogeneous subspace of $\left.\mathcal{H}_{\mathcal{X}}\right|_{Q_{i}=1}$. Because $\left.\mathcal{H}_{\mathcal{X}}^{-}\right|_{Q_{i}=1}$ is homogeneous and opposite to $\mathbb{E}_{\lim , \tau_{0}}^{\mathcal{X}}$, there is a graded isomorphism:

$$
H_{\text {orb }}^{\bullet}(\mathcal{X}) \cong z \mathcal{H}_{-}^{\mathcal{X}} /\left.\mathcal{H}_{-}^{\mathcal{X}}\right|_{Q_{i}=1} \cong \mathbb{E}_{\lim , \tau_{0}}^{\mathcal{X}} / z \mathbb{E}_{\text {lim }, \tau_{0}}^{\mathcal{X}}
$$

On the other hand, $\mathbb{U}\left(\mathbb{E}_{\lim , \tau_{0}}^{\mathcal{X}}\right)$ is also homogeneous since $\mathbb{U}$ is degree-preserving. Using Conjecture 5.1(c) again, there is a graded isomorphism:

$$
H^{\bullet}(Y) \cong z \mathcal{H}_{-}^{Y} /\left.\mathcal{H}_{-}^{Y}\right|_{Q_{i}=1} \cong \mathbb{U}\left(\mathbb{E}_{\lim , \tau_{0}}^{\mathcal{X}}\right) / z \mathbb{U}\left(\mathbb{E}_{\lim , \tau_{0}}^{\mathcal{X}}\right)
$$

The map $\mathbb{U}$ induces a graded isomorphism $\mathbb{E}_{\lim , \tau_{0}}^{\mathcal{X}} / z \mathbb{E}_{\lim , \tau_{0}}^{\mathcal{X}} \cong \mathbb{U}\left(\mathbb{E}_{\lim , \tau_{0}}^{\mathcal{X}}\right) / z \mathbb{U}\left(\mathbb{E}_{\lim , \tau_{0}}^{\mathcal{X}}\right)$, and so the conclusion follows.

\subsection{A Hard Lefschetz condition and the Bryan-Graber Conjecture}

Theorem 5.10 Suppose that Conjecture 5.1 holds, that $\mathcal{X}$ has complex dimension $n$, and that $\mathcal{X}$ in addition satisfies the Hard Lefschetz condition

$$
\omega^{i} \cup: H_{\mathrm{orb}}^{n-i}(\mathcal{X}) \rightarrow H_{\mathrm{orb}}^{n+i}(\mathcal{X}) \text { is an isomorphism for all } i \geq 0
$$

where $\omega \in H^{2}(\mathcal{X} ; \mathbb{C})$ is a Kähler class and $\cup$ is the Chen-Ruan orbifold cup product. Then $\mathbb{U}\left(\mathcal{H}_{\overline{\mathcal{X}}}^{-}\right)=\mathcal{H}_{Y}^{-}$.

In view of the discussion in Section 5.1, this implies:

${ }^{16}$ In fact the isomorphism we construct depends only on the equivalence class of $\tau_{0}$ in $H_{\text {orb }}^{2}(\mathcal{X} ; \mathbb{C}) / H^{2}(\mathcal{X} ; \mathbb{C})$. 
Corollary 5.11 Conjecture 5.1 implies the revised form of the Bryan-Graber Conjecture [8].

Proof of Theorem 5.10 We need to show that matrix elements of $\mathbb{U}$ do not contain strictly positive powers of $z$. Because $\mathbb{U}$ is a symplectic operator, the inverse of $\mathbb{U}$ is given by the adjoint $\mathbb{U}^{\dagger}$ with the sign of $z$ flipped. Thus it suffices to show that matrix elements of $\mathbb{U}^{-1}$ do not contain strictly positive powers of $z$. By taking a Jordan normal form of the nilpotent operator $\pi^{\star}(\omega) \cup$ on $H^{\bullet}(Y ; \mathbb{C})$, we obtain a basis for $H^{\bullet}(Y ; \mathbb{C})$ of the form

$$
\left\{\pi^{\star}(\omega)^{i} \phi_{j}: 1 \leq j \leq l, 0 \leq i \leq a_{j}\right\} \quad a_{1} \geq a_{2} \geq \cdots \geq a_{l}
$$

such that $\pi^{\star}(\omega)^{a_{j}+1} \phi_{j}=0$. We can assume that $\phi_{j}$ is homogeneous of degree $n-a_{j}+\lambda_{j}$ for some $\lambda_{j} \in \mathbb{Z}$. Since $\pi^{\star}(\omega)$ is conjugate to $\omega$ over $\mathbb{C}\left\{z, z^{-1}\right\}$, the Jordan normal forms of $\omega$ and $\pi^{\star}(\omega)$ are the same. The hard Lefschetz condition gives the Lefschetz decomposition of $H_{\mathrm{orb}}^{\bullet}(\mathcal{X})$ :

$$
H_{\mathrm{orb}}^{\bullet}(\mathcal{X})=\bigoplus_{i=0}^{n} \bigoplus_{k=0}^{i} \omega^{k} P H_{\mathrm{orb}}^{n-i}(\mathcal{X})
$$

where $P H_{\mathrm{orb}}^{n-i}(\mathcal{X})$ is the primitive cohomology group:

$$
P H_{\mathrm{orb}}^{n-i}(\mathcal{X})=\left\{\phi \in H_{\mathrm{orb}}^{n-i}(\mathcal{X}): \omega^{i+1} \phi=0\right\}
$$

The numbers $a_{j}$ above are determined by the Lefschetz decomposition. The variance $v_{\mathcal{X}}$ of the spectrum of $H_{\mathrm{orb}}^{\bullet}(\mathcal{X})$ is:

$$
v_{\mathcal{X}}=\sum_{i=0}^{2 n}(i-n)^{2} \operatorname{dim} H_{\mathrm{orb}}^{i}(\mathcal{X})=\sum_{j=1}^{l} \sum_{i=0}^{a_{j}}\left(-a_{j}+2 i\right)^{2}
$$

On the other hand, the variance $v_{Y}$ of $H^{\bullet}(Y)$ is:

$$
v_{Y}=\sum_{j=1}^{l} \sum_{i=0}^{a_{j}}\left(-a_{j}+\lambda_{j}+2 i\right)^{2}=v_{\mathcal{X}}+\sum_{j=1}^{l}\left(1+a_{j}\right) \lambda_{j}^{2}
$$

Since there is a graded isomorphism $H_{\mathrm{orb}}^{\bullet}(\mathcal{X}) \cong H^{\bullet}(Y)$, we have $v_{\mathcal{X}}=v_{Y}$ and so $\lambda_{j}=0$ for all $j$. Thus $\operatorname{deg} \phi_{j}=n-a_{j}$. Then $\mathbb{U}^{-1}\left(\phi_{j}\right)$ is in $\operatorname{Ker}\left(\omega^{a_{j}+1}\right)$ and is also of degree $n-a_{j}$. Using the Lefschetz decomposition of $H_{\text {orb }}^{\bullet}(\mathcal{X} ; \mathbb{C})$ again, we see that $\mathbb{U}^{-1}\left(\phi_{j}\right)$ does not contain positive powers of $z$. Thus $\mathbb{U}^{-1}\left(\pi^{\star}(\omega)^{i} \phi_{j}\right)=\omega^{i} \mathbb{U}^{-1}\left(\phi_{j}\right)$ does not contain positive powers of $z$ either. 
Remark 5.12 Fernandez [22] has shown that the Hard Lefschetz condition in Theorem 5.10 is equivalent to the equality age $\mathcal{X}_{i}=$ age $I\left(\mathcal{X}_{i}\right)$ for all components $\mathcal{X}_{i}$ of the inertia stack $\mathcal{I} \mathcal{X}$. This condition holds for $\mathbb{P}(1,1,2)$ and for any other two-dimensional Gorenstein orbifold but not for $\mathbb{P}(1,1,1,3)$.

Remark 5.13 Since the paper was written, Iritani [37, Section 3.7] has studied a more general form of the Hard Lefschetz condition, which is applicable to partial resolutions and $K$-equivalences.

\subsection{Conjecture 5.1 and the Ruan Conjecture}

As we have seen in Theorems 1.1 and 1.2, by proving Conjecture 5.1 we also proved the Ruan Conjecture for $\mathcal{X}=\mathbb{P}(1,1,2)$ and $\mathcal{X}=\mathbb{P}(1,1,1,3)$. This is slightly misleading, however, as in general our Conjecture only implies a modified version of the Ruan Conjecture. This is explained in detail in Coates-Ruan [17, Section 8]. Coates [13] has proved our Conjecture, and hence the modified Ruan Conjecture, in an example for which the modified Ruan Conjecture and the original Ruan Conjecture differ: this example is the canonical bundle to $\mathbb{P}(1,1,3)$. We expect that the original version of the Ruan Conjecture is false in general.

\subsection{Open questions}

We close by indicating several questions which deserve further study. One such direction involves real and integral structures ${ }^{17}$ on the VSHS. The B-model VSHS has a natural integral structure, coming from the lattice of Morse cycles, but this is hard to see in the A-model. The study of real structures should lead to $t t^{*}$-geometry of the Kähler moduli space (see Cecotti-Vafa [10], Dubrovin [20], and Hertling [30]). There should also be a hidden real structure on Givental's symplectic space $\mathcal{H}$, and the symplectic transformation $\mathbb{U}$ from Conjecture 5.1 should preserve such real structures. The specialization of Kähler parameters to purely imaginary numbers (such as $\tau^{1}=2 \pi i$, $\tau^{2}=-\pi i+\frac{1}{2} \tau$ in the case $\left.\mathcal{X}=\mathbb{P}(1,1,2), Y=\mathbb{F}_{2}\right)$ might be explained from this viewpoint, as they can be read off from $\mathbb{U}(1)$.

One should also consider higher-genus Gromov-Witten invariants. In Givental's quantization formalism [28], the total descendant potential corresponds to a quantization of the (genus-zero) Lagrangian cones and lives in a Fock space produced from $\mathcal{H}$. This suggests that the total descendant potentials of $\mathcal{X}$ and $Y$ are related by

$$
\mathcal{D}_{Y} \propto \widehat{\mathbb{U}} \mathcal{D}_{\mathcal{X}}
$$

\footnotetext{
${ }^{17}$ Since this paper was written, this question has been studied by Iritani [36].
} 
We do not give rigorous meaning to this formula here; the idea is that the two elements $\mathcal{D}_{Y}, \mathcal{D}_{\mathcal{X}}$ of different Fock spaces will be projectively identified by the change of polarization $\mathbb{U}$. More than a decade ago, Witten [50] introduced a quantum mechanical system on $H^{3}(X)$ for a Calabi-Yau threefold $X$ and showed that the total potential of the B-model behaves like a wave function of this quantum system. Formula (74) fits with this picture. It again matches well with the ideas of Ruan discussed above, and also with recent work of Aganagic, Bouchard and Klemm [3]. They argue that the fundamental group of the B-model moduli space should act as "quantum symmetries" of the total descendant potential. The monodromy around the large radius limit point is, as discussed in Remark 5.3, related to the Divisor Equation in Gromov-Witten theory. When we have a crepant resolution $Y \rightarrow X$ of $\mathcal{X}$, there should also be an "extra" monodromy action on the Gromov-Witten theory of $Y$ coming from orbifold loops around the large radius limit point for $\mathcal{X}$; such monodromy will not in general preserve the opposite subspace $\mathcal{H}_{Y}^{-}$for $Y$. We hope that these symmetries together with a hidden integral structure will reveal a kind of quantum automorphic property of the potential $\mathcal{D}_{Y}$.

\section{Appendix}

In this appendix we give a brief account of the analytic continuation of the $I$-function performed in (52). We use an integral representation of Barnes type, following Candelas et al [9], Horja [35] and Borisov-Horja [7].

Set $\mathfrak{p}_{1}=p_{2}-3 p_{1}$, as in Figure 2. During the analytic continuation we regard $p_{1}$ and $p_{2}$ as complex variables and consider the $I$-function as an analytic function in $y_{1}$, $y_{2}, p_{1}, p_{2}$, and $z$. We obtain cohomology classes by, at the end of the process, Taylor expanding in $p_{1}$ and $p_{2}$ and then regarding $p_{1}$ and $p_{2}$ as cohomology classes. We have:

$$
\begin{aligned}
I_{\mathbb{F}_{3}}\left(y_{1}, y_{2}, z\right)= & z \Gamma\left(1+\frac{p_{1}}{z}\right)^{3} \Gamma\left(1+\frac{p_{2}}{z}\right) \Gamma\left(1+\frac{\mathfrak{p}_{1}}{z}\right) \\
& \times \sum_{n, m \geq 0} \frac{y_{1}^{n+p_{1} / z} y_{2}^{m+p_{2} / z} z^{-2 m}}{\Gamma\left(1+\frac{p_{1}}{z}+n\right)^{3} \Gamma\left(1+\frac{p_{2}}{z}+m\right) \Gamma\left(1+\frac{\mathfrak{p}_{1}}{z}+m-3 n\right)}
\end{aligned}
$$

Since $\Gamma(z) \Gamma(1-z)=\pi / \sin (\pi z)$ the coefficient of $y_{2}^{m+p_{2} / z}$ in (75) can be written, neglecting several Gamma factors and powers of $z$, as:

$$
\sum_{n \geq 0} \frac{\Gamma\left(-\frac{\mathfrak{p}_{1}}{z}+3 n-m\right)}{\Gamma\left(1+\frac{p_{1}}{z}+n\right)^{3}} \frac{\sin \left(-\frac{\mathfrak{p}_{1}}{z} \pi+3 n \pi-m \pi\right)}{\pi} y_{1}^{n+p_{1} / z}
$$


This is the sum of residues:

$$
(-1)^{m} \frac{\sin \left(-\frac{\mathfrak{p}_{1}}{z} \pi\right)}{\pi} \sum_{n \geq 0} \operatorname{Res}_{s=n}\left[\Gamma(s) \Gamma(1-s) \frac{\Gamma\left(-\frac{\mathfrak{p}_{1}}{z}+3 s-m\right)}{\Gamma\left(1+\frac{p_{1}}{z}+s\right)^{3}} y_{1}^{s+p_{1} / z} d s\right]
$$

For $\left|y_{1}\right|<\frac{1}{27}$, it can be replaced by the integral along a contour $C_{m}$ from $s=i \infty$ to $s=-i \infty$ which runs along the imaginary axis for $|s|$ large and is such that $s=0,1,2, \ldots$ are on the right hand side of $C_{m}$ and that $s=-1,-2,-3, \ldots$ and $s=\frac{m}{3}+\frac{\mathfrak{p}_{1}}{3 z}, \frac{m}{3}+\frac{\mathfrak{p}_{1}}{3 z}-\frac{1}{3}, \frac{m}{3}+\frac{\mathfrak{p}_{1}}{3 z}-\frac{2}{3}, \ldots$ are on the left hand side of $C_{m}$ :

$$
(-1)^{m} \frac{\sin \left(-\frac{\mathfrak{p}_{1}}{z} \pi\right)}{\pi} \frac{1}{2 \pi i} \int_{C_{m}} \frac{\Gamma\left(-\frac{\mathfrak{p}_{1}}{z}+3 s-m\right) \Gamma(s) \Gamma(1-s)}{\Gamma\left(1+\frac{p_{1}}{z}+s\right)^{3}} y_{1}^{s+p_{1} / z} d s
$$

This integral converges on the region $\left|\arg \left(y_{1}\right)\right|<\pi$; see for example Borisov-Horja [7, Lemma A.6]. For $\left|y_{1}\right|>\frac{1}{27}$ we can close the contour to the left, finding:

$$
\begin{aligned}
& (-1)^{m} \frac{\sin \left(\frac{\mathfrak{p}_{1}}{z} \pi\right)}{\pi} \sum_{n \geq 0} \operatorname{Res}_{s=\frac{m}{3}+\frac{\mathfrak{p}_{1}}{3 z}-\frac{n}{3}}\left[\frac{\Gamma\left(-\frac{\mathfrak{p}_{1}}{z}+3 s-m\right) \Gamma(s) \Gamma(1-s)}{\Gamma\left(1+\frac{p_{1}}{z}+s\right)^{3}} y_{1}^{s+p_{1} / z} d s\right] \\
& +(-1)^{m} \frac{\sin \left(\frac{\mathfrak{p}_{1}}{z} \pi\right)}{\pi} \sum_{n \geq 0} \operatorname{Res}_{s=-1-n}\left[\frac{\Gamma\left(-\frac{\mathfrak{p}_{1}}{z}+3 s-m\right) \Gamma(s) \Gamma(1-s)}{\Gamma\left(1+\frac{p_{1}}{z}+s\right)^{3}} y_{1}^{s+p_{1} / z} d s\right]
\end{aligned}
$$

The residues at $s=-1-n$ vanish in cohomology, as $p_{1}^{3}=0$, so this is:

$$
\sum_{n \geq 0} \frac{(-1)^{m+n}}{n !} \frac{\sin \left(\frac{\mathfrak{p}_{1}}{z} \pi\right)}{3 \sin \left(\frac{\mathfrak{p}_{1}}{3 z} \pi+\frac{m-n}{3} \pi\right)} \frac{y_{1}^{(m-n) / 3+p_{2} /(3 z)}}{\Gamma\left(1+\frac{p_{2}}{3 z}+\frac{m-n}{3}\right)^{3}}
$$

Changing variables from $\left(y_{1}, y_{2}\right)$ to $\left(\mathfrak{y}_{1}, \mathfrak{y}_{2}\right)$ yields $(52)$.

\section{References}

[1] D Abramovich, T Graber, A Vistoli, Algebraic orbifold quantum products, from: "Orbifolds in mathematics and physics (Madison, WI, 2001)", (A Adem, J Morava, Y Ruan, editors), Contemp. Math. 310, Amer. Math. Soc. (2002) 1-24 MR1950940

[2] D Abramovich, T Graber, A Vistoli, Gromov-Witten theory of Deligne-Mumford stacks, Amer. J. Math. 130 (2008) 1337-1398 MR2450211

[3] M Aganagic, V Bouchard, A Klemm, Topological strings and (almost) modular forms, Comm. Math. Phys. 277 (2008) 771-819 MR2365453

[4] M Audin, Torus actions on symplectic manifolds, revised edition, Progress in Math. 93, Birkhäuser Verlag, Basel (2004) MR2091310 
[5] S Barannikov, Semi-infinite Hodge structures and mirror symmetry for projective spaces arXiv:math.AG/0010157

[6] S Barannikov, Quantum periods. I. Semi-infinite variations of Hodge structures, Internat. Math. Res. Notices (2001) 1243-1264 MR1866443

[7] L A Borisov, R P Horja, Mellin-Barnes integrals as Fourier-Mukai transforms, Adv. Math. 207 (2006) 876-927 MR2271990

[8] J Bryan, T Graber, The crepant resolution conjecture, from: "Algebraic geometrySeattle 2005. Part 1”, (D Abramovich, A Bertram, L Katzarkov, R Pandharipande, M Thaddeus, editors), Proc. Sympos. Pure Math. 80, Amer. Math. Soc. (2009) 23-42 MR2483931

[9] P Candelas, X C de la Ossa, P S Green, L Parkes, A pair of Calabi-Yau manifolds as an exactly soluble superconformal theory, Nuclear Phys. B 359 (1991) 21-74 MR1115626

[10] S Cecotti, C Vafa, On classification of $N=2$ supersymmetric theories, Comm. Math. Phys. 158 (1993) 569-644 MR1255428

[11] W Chen, Y Ruan, Orbifold Gromov-Witten theory, from: "Orbifolds in mathematics and physics (Madison, WI, 2001)", (A Adem, J Morava, Y Ruan, editors), Contemp. Math. 310, Amer. Math. Soc. (2002) 25-85 MR1950941

[12] W Chen, Y Ruan, A new cohomology theory of orbifold, Comm. Math. Phys. 248 (2004) 1-31 MR2104605

[13] T Coates, On the crepant resolution conjecture in the local case, Comm. Math. Phys. 287 (2009) 1071-1108 MR2486673 A longer version with more examples: Wallcrossings in toric Gromov-Witten theory II: Local examples arxiv : 0804.2592

[14] T Coates, A Corti, H Iritani, H-H Tseng, Computing genus-zero twisted GromovWitten invariants, Duke Math. J. 147 (2009) 377-438 MR2510741

[15] T Coates, A Corti, Y-P Lee, H-H Tseng, The quantum orbifold cohomology of weighted projective spaces, Acta Math. 202 (2009) 139-193

[16] T Coates, A B Givental, Quantum Riemann-Roch, Lefschetz and Serre, Ann. of Math. (2) 165 (2007) 15-53 MR2276766

[17] T Coates, Y Ruan, Quantum cohomology and crepant resolutions: A conjecture arXiv:0710.5901v3

[18] D A Cox, S Katz, Mirror symmetry and algebraic geometry, Math. Surveys and Monogr. 68, Amer. Math. Soc. (1999) MR1677117

[19] A Douai, C Sabbah, Gauss-Manin systems, Brieskorn lattices and Frobenius structures. I, from: "Proceedings of the International Conference in Honor of Frédéric Pham (Nice, 2002)", Ann. Inst. Fourier (Grenoble) 53 (2003) 1055-1116 MR2033510

[20] B Dubrovin, Geometry and integrability of topological-antitopological fusion, Comm. Math. Phys. 152 (1993) 539-564 MR1213301

[21] B Dubrovin, Geometry of 2D topological field theories, from: "Integrable systems and quantum groups (Montecatini Terme, 1993)", (M Francaviglia, S Greco, editors), Lecture Notes in Math. 1620, Springer, Berlin (1996) 120-348 MR1397274 
[22] J Fernandez, Hodge structures for orbifold cohomology, Proc. Amer. Math. Soc. 134 (2006) 2511-2520 MR2213728

[23] W Fulton, R Pandharipande, Notes on stable maps and quantum cohomology, from: “Algebraic geometry-Santa Cruz 1995”, (J Kollár, R Lazarsfeld, D R Morrison, editors), Proc. Sympos. Pure Math. 62, Amer. Math. Soc. (1997) 45-96 MR1492534

[24] A B Givental, Homological geometry and mirror symmetry, from: "Proceedings of the International Congress of Mathematicians, Vol. 1, 2 (Zürich, 1994)”, Birkhäuser, Basel (1995) 472-480 MR1403947

[25] A B Givental, Homological geometry. I. Projective hypersurfaces, Selecta Math. (N.S.) 1 (1995) 325-345 MR1354600

[26] A B Givental, A mirror theorem for toric complete intersections, from: "Topological field theory, primitive forms and related topics (Kyoto, 1996)", (M Kashiwara, A Matsuo, K Saito, I Satake, editors), Progr. Math. 160, Birkhäuser, Boston (1998) 141-175 MR1653024

[27] A B Givental, Gromov-Witten invariants and quantization of quadratic Hamiltonians, Mosc. Math. J. 1 (2001) 551-568, 645 MR1901075 Dedicated to the memory of I G Petrovskii on the occasion of his 100th anniversary

[28] A B Givental, Symplectic geometry of Frobenius structures, from: "Frobenius manifolds", (C Hertling, M Marcolli, editors), Aspects Math. E36, Vieweg, Wiesbaden (2004) 91-112 MR2115767

[29] MA Guest, Quantum cohomology via D-modules, Topology 44 (2005) 263-281 MR2114708

[30] C Hertling, $t t^{*}$ geometry, Frobenius manifolds, their connections, and the construction for singularities, J. Reine Angew. Math. 555 (2003) 77-161 MR1956595

[31] C Hertling, Y Manin, Weak Frobenius manifolds, Internat. Math. Res. Notices (1999) 277-286 MR1680372

[32] C Hertling, Y Manin, Unfoldings of meromorphic connections and a construction of Frobenius manifolds, from: "Frobenius manifolds", (C Hertling, M Marcolli, editors), Aspects Math. E36, Vieweg, Wiesbaden (2004) 113-144 MR2115768

[33] K Hori, S Katz, A Klemm, R Pandharipande, R Thomas, C Vafa, R Vakil, E Zaslow, Mirror symmetry, Clay Math. Monogr. 1, Amer. Math. Soc. (2003) MR2003030

[34] K Hori, C Vafa, Mirror symmetry arXiv:hep-th/0002222

[35] P R Horja, Hypergeometric functions and mirror symmetry in toric varieties arXiv: math. AG/9912109

[36] H Iritani, An integral structure in quantum cohomology and mirror symmetry for toric orbifolds, to appear in Adv. Math A longer version containing a discussion on real structures: Real and integral structures in quantum cohomology I: Toric orbifolds arxiv:0712.2204

[37] H Iritani, Ruan's conjecture and integral structures in quantum cohomology arXiv: 0809.2749

[38] H Iritani, Wall-crossings in toric Gromov-Witten theory III, in preparation 
[39] H Iritani, Quantum D-modules and generalized mirror transformations, Topology 47 (2008) 225-276 MR2416770

[40] M Kontsevich, Y Manin, Gromov-Witten classes, quantum cohomology, and enumerative geometry, Comm. Math. Phys. 164 (1994) 525-562 MR1291244

[41] B H Lian, K Liu, S-T Yau, Mirror principle. II, Asian J. Math. 3 (1999) 109-146 MR1701925

[42] E Lupercio, M Poddar, The global McKay-Ruan correspondence via motivic integration, Bull. London Math. Soc. 36 (2004) 509-515 MR2069013

[43] Y I Manin, Frobenius manifolds, quantum cohomology, and moduli spaces, Amer. Math. Soc. Coll. Publ. 47, Amer. Math. Soc. (1999) MR1702284

[44] A Pressley, G Segal, Loop groups, Oxford Math. Monogr., Oxford Science Publ., The Clarendon Press, Oxford Univ. Press, New York (1986) MR900587

[45] M A Rose, A reconstruction theorem for genus zero Gromov-Witten invariants of stacks, Amer. J. Math. 130 (2008) 1427-1443 MR2450213

[46] Y Ruan, private communication

[47] K Saito, Period mapping associated to a primitive form, Publ. Res. Inst. Math. Sci. 19 (1983) 1231-1264 MR723468

[48] M Saito, On the structure of Brieskorn lattice, Ann. Inst. Fourier (Grenoble) 39 (1989) 27-72 MR1011977

[49] W Schmid, Variation of Hodge structure: the singularities of the period mapping, Invent. Math. 22 (1973) 211-319 MR0382272

[50] E Witten, Quantum background independence in string theory arXiv: hep-th/9306122

[51] T Yasuda, Twisted jets, motivic measures and orbifold cohomology, Compos. Math. 140 (2004) 396-422 MR2027195

Department of Mathematics, Imperial College London

180 Queen's Gate, London SW7 2AZ, UK

Faculty of Mathematics, Kyushu University

6-10-1, Hakozaki, Higashiku, Fukuoka, 812-8581, Japan

Department of Mathematics, University of Wisconsin-Madison

Van Vleck Hall, 480 Lincoln Drive, Madison, WI 53706-1388, USA

t.coates@imperial.ac.uk, iritani@math.kyushu-u.ac.jp, tseng@math.wisc.edu

Proposed: Jim Bryan

Seconded: Richard Thomas, Lothar Goettsche
Received: 4 December 2006

Revised: 21 October 2008 